\title{
Dorcadionini of Turkey (Coleoptera: Cerambycidae)
}

\author{
Hüseyin Özdikmen
}

Science Faculty, Department of Biology, Gazi University, Ankara, Turkey

\begin{abstract}
The Dorcadionini fauna of Turkey is reviewed, and a complete list with type information and provincial distributions is presented. Two hundred and seventy-eight species-group taxa are catalogued in total. This represents approximately $35 \%$ of the known Palaearctic Dorcadionini fauna. The Turkish Dorcadionini fauna is characterised by 227 endemic species-group taxa. So its endemism ratio is very high (approximately $82 \%$ ). Distributions of the taxa in Turkey are given based on available literature records. Taxa in the list are arranged systematically under tribe, genus, subgenus (when recognised), species and subspecies (when recognised). The species group taxa whose occurrences in Turkey need conformation are not included in the list but are given separately. Additionally, two taxa are removed from the list: Dorcadion (Cribridorcadion) investitum Breuning in Braun, 1978a as nomen nudum and Dorcadion (Cribridorcadion) rosinae K. Daniel in Bodemeyer, 1900 as nomen nudum; and two taxa are given as status nov. and comb. nov.: Dorcadion (Cribridorcadion) atritarse Pic, 1931a and Dorcadion (Cribridorcadion) equestre bernhauerorum Peks, 2010. Moreover, a checklist of Dorcadionini of Turkey is presented in an appendix.
\end{abstract}

\section{ARTICLE HISTORY}

Received 18 September 2015

Accepted 20 May 2016

\section{KEYWORDS}

Coleoptera; Cerambycidae;

Dorcadionini; list; Turkey

\section{Introduction}

Turkey is adjacent to large bodies of water to the south, west and north; it has continental properties. Turkey is the centre of origin of many taxa, and its exceptionally diverse topography has provided refugia in which many species have survived in spite of harsh geological and climatic changes. The great biological importance of Turkey is evident from the remarkable variety of arthropods in Turkey. Nevertheless the fauna of Cerambycidae of Turkey have not been thoroughly studied and documented (Özdikmen and Koçak 2015).

Also, Turkey is one of the most diverse areas of the west Palaearctic region with its unique geographic position as a land bridge between Asia, Europe and North Africa (also linking to the Ethiopian Region via the Arabian Peninsula) and accumulating natural complexes typical of several climatic zones. Three of four hotspots of Europe and Central Asia recognised by Conservation International are partly included within the boundary of Turkey: Caucasus, Irano-Anatolian and Mediterranean Basin hotspots.

CONTACT Hüseyin Özdikmen ozdikmen@gazi.edu.tr Science Faculty, Department of Biology, Gazi University, 06500 Ankara, Turkey 
Hence, Turkey contains a rich flora and fauna with a considerable number of endemic species (Myers et al. 2000; Çıplak 2003, 2004; Konstantinov et al. 2009; Ekiz et al. 2013; Conservation International c. 2015).

The aim of this work is to determine the current status of the tribe Dorcadionini in Turkey. This work on Dorcadionini of Turkey is meant to realise a study on approximately one-third of the Turkish Cerambycidae fauna. By the same token, the Turkish Dorcadionini represent more than one-third of the Dorcadionini in the whole world.

Hence, Dorcadionini of Turkey is a very significant group. This importance originates from its high endemism ratio (approximately $82 \%$ ), and also the large number of speciesgroup taxa (278). However, the information on all Turkish Dorcadionini is not sufficient.

The first large work on Dorcadionini species was published by Thomson (1867). Another large work on Dorcadionini species was carried out by Ganglbauer (1884). He evaluated a total of 152 species (30 species for Turkey) of the genus Dorcadion and 13 species of the genus Neodorcadion (four species for Turkey). Then, the number of Turkish Dorcadion species exceeded 50 with species described by various authors. In 1962, the largest work on Dorcadionini was accomplished by Breuning. He mentioned 353 Dorcadion species (92 species for Turkey) and nine Neodorcadion species (five species for Turkey) in his study. After Breuning (1962a), many new Dorcadion species had been described by various authors from Turkey until 1990. In 1990 and 1991, a study on Turkish Dorcadion fauna was carried out by Önalp. She stated a total of 52 species for Turkey. However, her work was far from determining the real status of Turkish Dorcadionini since it lacked a great deal of information for Turkey. After Önalp's work, many new Dorcadion species were also described by various authors from Turkey. Recently, Löbl and Smetana (2010) recorded a total of 155 species for Dorcadionini of Turkey while Özdikmen (2010) reported a total of 192 species for Turkey.

According to Breuning (1962a), the tribe Dorcadionini included only the genera Dorcadion Dalman, 1817, Neodorcadion Ganglbauer, 1884 and Eodorcadion Breuning, 1947. Iberodorcadion described by Breuning (1943) as a subgenus of the genus Dorcadion, was ranked by Vives (1976) as a separate genus. Also, Danilevsky et al. (2005) accepted it as a genus on the basis of endophallic structures. So, recently, Löbl and Smetana (2010) and Danilevsky (2015a) listed it as a separate genus. Politodorcadion was described by Danilevsky (1996) as a subgenus of Dorcadion. Recently, Löbl and Smetana (2010) and Danilevsky (2015a) listed it as a separate genus. The genus Trichodorcadion Breuning, 1942 from India and Nepal was transfered in Dorcadionini by Danilevsky and Kasatkin (2007) on the base of endophallic structures. However, Löbl and Smetana (2010) and Danilevsky (2015a) gave Trichodorcadion in the tribe Morimopsini. As a result of this, Dorcadionini included five genera as Dorcadion Dalman, 1817, Neodorcadion Ganglbauer, 1884, Iberodorcadion Breuning, 1943, Eodorcadion Breuning, 1947 and Politodorcadion Danilevsky (1996). Recently, Özdikmen and Kaya (2015g) proposed new arrangements for some Dorcadionini. After this work, the tribe Dorcadionini includes six genera and 17 subgenera (including nominate subgenera) worldwide now.

The tribe Dorcadionini has a Palaearctic (North Africa and Western Europe to China) chorotype. Iberodorcadion Breuning, 1943 has a European (chiefly Iberian) chorotype (Spain to Poland). Eodorcadion Breuning, 1947 has an East Palaearctic chorotype (Russia, Siberia, Mongolia and China). Politodorcadion Danilevsky (1996) has an Asian chorotype 
(Kazakhstan, West Siberia and China). These genera are not represented in Turkey. After Özdikmen and Kaya (2015g), the tribe Dorcadionini of Turkey is represented by the genera Dorcadion Dalman, 1817, Megalodorcadion Pesarini and Sabbadini, 1998 and Neodorcadion Ganglbauer, 1884.

\section{Material and methods}

This list is the consequence of careful reviews of the available literature on Turkish Dorcadionini. The genera, subgenera, species and subspecies are listed alphabetically. Information in the list is as follows: the name of the species or subspecies and author(s), type information, synonyms, provincial distributions in Turkey, the literature(s) in which the species or subspecies is cited (in parentheses), and distribution in the world. The type information for each species or subspecies is based on Tavakilian (2015). The provincial distributions of taxa in Turkey are coded as in Table 1. A map showing the administrative provinces of Turkey is provided (Figure 1). It was not possible to give the exact provinces of some species or subspecies, so they are recorded as Asian Turkey (TRA) or European Turkey (TR-E). For global distributional data, Löbl and Smetana (2010) and Danilevsky (2015a) are chiefly used in the text. Distributional abbreviations for the work are available in Löbl and Smetana (2010). The endemic species are marked with an asterisk. A checklist of Dorcadionini of Turkey is given in Appendix.

Table 1. Abbreviations of localities (administrative divisions) of Turkey used in the text and in Figure 1.

\begin{tabular}{lll}
\hline Adana (AD) & Erzincan (ER) & Muğla (MG) \\
Adiyaman (ADY) & Erzurum (EZ) & Muş (MU) \\
Afyon (AF) & Eskişehir (ES) & Nevşehir (NE) \\
Ağri (AG) & Gaziantep (GA) & Niğde (NI) \\
Aksaray (AK) & Giresun (GI) & Ordu (OR) \\
Amasya (AM) & Gümüşhane (GU) & Osmaniye (OS) \\
Ankara (AN) & Hakkâri (HA) & Rize (RI) \\
Antalya (ANT) & Hatay (HT) & Sakarya (SA) \\
Ardahan (AR) & Iğdir (IG) & Samsun (SM) \\
Artvin (ART) & Isparta (IP) & Siirt (SI) \\
Aydin (AY) & İçel (IC) & Sinop (SN) \\
Balikesir (BL) & İstanbul (IS) & Sivas (SV) \\
Bartin (BR) & Izmir (IZ) & Şanliurfa (SU) \\
Batman (BA) & Kahramanmaraş (KA) & Şirnak (SK) \\
Bayburt (BY) & Karabük (KR) & Tekirdağ (TE) \\
Bilecik (BI) & Karaman (KM) & Tokat (TO) \\
Bingöl (BN) & Kars (KAR) & Trabzon (TB) \\
Bitlis (BT) & Kastamonu (KS) & Tunceli (TU) \\
Bolu (BO) & Kayseri (KY) & Uşak (US) \\
Burdur (BU) & Kirikkale (KI) & Van (VA) \\
Bursa (BS) & Kirklareli (KK) & Yalova (YA) \\
Çanakkale (CA) & Kirşehir (KIR) & Yozgat (YO) \\
Çankiri (CN) & Kilis (KL) & Zonguldak (ZO) \\
Çorum (CO) & Malatya (MA) & European \\
Denizli (DE) & Manisa (MN) & Turkey = Thracia (TR-E) \\
Diyarbakir (DI) & Mardin (MR) & Asian \\
Düzce (DU) & Kocaeli (KO) & Turkey = Anatolia (TR-A) \\
Edirne (ED) & Konya (KN) & Turkey (TR) \\
Elaziğ (EL) & Kütahya (KU) & \\
\hline & & \\
\hline
\end{tabular}




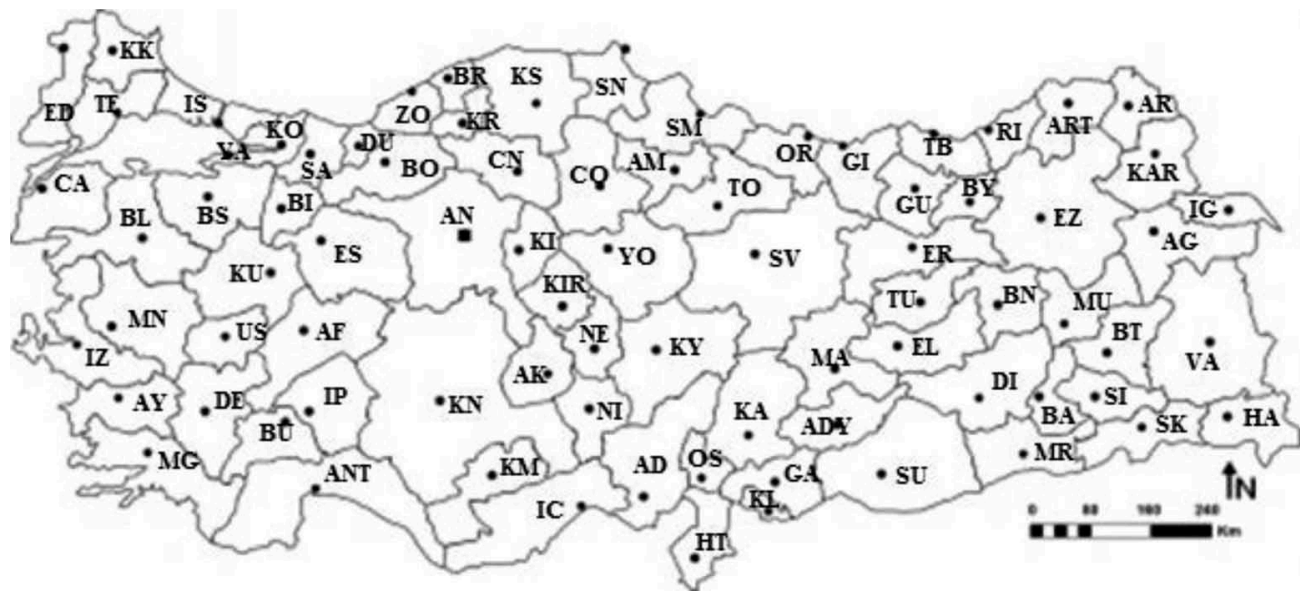

Figure 1. Map showing the administrative provinces of Turkey.

\section{Taxonomy}

All species-group taxa of the Dorcadionini of Turkey are presented as follows:

Tribe DORCADIONINI Swainson and Shuckard, 1840

Genus DORCADION Dalman, 1817: 397

[type species Cerambyx glicyrrhizae Pallas, 1773]

Subgenus D. (CARINATODORCADION) Breuning, 1943: 524

[type species Cerambyx carinatus Pallas, 1771]

D. aethiops Scopoli, 1763: 53 (Cerambyx)

D. aethiops aethiops Scopoli, 1763: 53 (Cerambyx)

Type information: Holotype, ex collection G.A. Scopoli [type locality 'Carniola: Circa Labacum (Ljubljana)' (Slovenia)]

morio Fabricius, 1787: 141 (Lamia) [Austria]

scopolii Gmelin, 1790: 1835 (Cerambyx) [Austria]

bergmanni Tippmann, 1958: 151 (D. aethiops ssp.) [Austria]

Provincial distribution in Turkey: TR-A: ES; TR-E: ED (Önalp 1990; Pesarini and Sabbadini 2007; Özdikmen 2010; Danilevsky 2015a)

Range: E (Europe): AL AU BU CR CZ GR HU MC MD RO SK SL TR UK YU A (Asia): TR Other known subspecies: D. aethiops asenovi Danilevsky, 2014: $253 \mathrm{E}: \mathrm{BU}$; D. aethiops majoripenne Pic, 1926: 13 E: GR; D. aethiops propinquum Breuning, 1962a: 517 E: GR; D. aethiops strumense Danilevsky, 2014: $252 \mathrm{E}: \mathrm{BU}$

\section{D. fulvum Scopoli, 1763: 53 (Cerambyx)}

D. fulvum erythropterum Fischer von Waldheim, 1823a: table L-7 (D. erythropteron)

Type information: Holotype $\hat{\sigma}$, ex collection Gotthelf Fischer von Waldheim, Zoological Museum, University of Moscow [type locality 'Rossia meridionalis' (Russia)]

canaliculatum Fischer von Waldheim, 1823b: 240 [S Russia]

frontale Mulsant and Rey, 1863: 154 [Turkey: Istanbul]

Provincial distribution in Turkey: TR-E: IS (Mulsant and Rey 1863; Özdikmen 2010;

Danilevsky 2015a) 
Range: E: AL BH BU CR MC MD RO PL TR UK YU

Other known subspecies: D. fulvum fulvum Scopoli, 1763: 53 (Cerambyx) E: AU CZ HU SK SL UK

D. hybridum Ganglbauer, 1884: 441

D. hybridum hybridum Ganglbauer, 1884: 441

Type information: Holotype $\hat{\jmath}$, Magyar Természettudományi Mûzeum, Budapest [type locality 'Rumelia' (Greece)]

Provincial distribution in Turkey: TR-E (Breuning 1962a; Althoff and Danilevsky 1997; Löbl and Smetana 2010; Özdikmen 2010; Danilevsky 2015a)

Range: E: GR TR

Other known subspecies: D. hybridum hedvigae Jureček, 1933: 127 E: GR

*D. ingeae Peks, 1993: 3

Type information: Holotype $\hat{\jmath}$, collection Heinz Peks, Schwanfeld [type locality 'Keşan' (Turkey: Edirne)]

Provincial distribution in Turkey: TR-E: ED (Peks 1993; Althoff and Danilevsky 1997; Löbl and Smetana 2010; Özdikmen 2010, 2012; Danilevsky 2015a)

Range: E: TR

Subgenus D. (Cribridorcadion) Pic, 1901: 12

[type species Dorcadion mniszechi Kraatz, 1873]

Pedestredorcadion Breuning, 1943: 526 [type species Lamia pedestris Poda von Neuhaus, 1761]

Dzhungarodorcadion Danilevsky, 1993: 47 [type species Dorcadion jacobsoni Jakovlev, 1899]

Autodorcadion Plavilstshikov, 1958: 45 [type species Cerambyx arenarius Scopoli, 1763]

Bergerianum Pesarini and Sabbadini, 2004: 150 [type species Dorcadion chrysochroum Breuning, 1943]

*D. abstersum Holzschuh, 1982: 75

Type information: Holotype $\hat{\jmath}$, collection Carolus Holzschuh, Villach [type locality 'Sat Mt.' (Turkey: Hakkari)]

Provincial distribution in Turkey: TR-A: HA (Holzschuh 1982; Löbl and Smetana 2010; Özdikmen 2010, 2012; Danilevsky 2015a)

Range: A: TR

*D. accola Heyden, 1894: 87

Type information: Holotype, ex collection L. von Heyden, Natur-Museum und Forschungs-Institut Senckenberg, Frankfurt am Main [type locality 'Mardin' (Turkey)] glabrolineatum Pic, 1927: 6 [?Macedonia]

Provincial distribution in Turkey: TR-A: MR SU (Heyden 1894; Breuning 1962a; Braun 1978a; Önalp 1991; Löbl and Smetana 2010; Özdikmen 2010, 2012; Danilevsky 2015a) Range: A: TR

*D. afflictum Pesarini and Sabbadini, 1998: 54

Type information: Holotype $\hat{\partial}$, collection Carlo Pesarini and Andrea Sabbadini, Milano [type locality 'Çorum' (Turkey)] 
Provincial distribution in Turkey: TR-A: CO (Pesarini and Sabbadini 1998; Löbl and Smetana 2010; Özdikmen 2010, 2012; Danilevsky 2015a)

Range: A: TR

*D. akpinarense Bernhauer and Peks, 2010: 196

Type information: Holotype $\widehat{\partial}$, collection Dieter Bernhauer, Bad Schwalbach [type locality 'Anamur: Akpınar' (Turkey: İçel)]

Provincial distribution in Turkey: TR-A: IC (Bernhauer and Peks 2010; Özdikmen 2012; Danilevsky 2015a)

Range: A: TR

*D. albolineatum Küster, 1847: 86

Type information: Holotype, ex collection Jacob Sturm, Zoologische Staatssammlung des Bayerischen Staates, München [type locality 'Istanbul' (Turkey)]

byzantinum J. Thomson, 1867: 69 [Turkey]

abbreviatolineatum Breuning, 1946: 132 (D. albolineatum m.) [Turkey]

Provincial distribution in Turkey: TR-A: BI DI IS KO (Küster 1847; Kraatz 1873; Breuning 1962a; Tuatay et al. 1972; Özdikmen et al. 2005; Löbl and Smetana 2010; Özdikmen 2010, 2012; Danilevsky 2015a)

Range: A: TR

*D. albonotatum Pic, 1895a: 39

Type information: Holotype, ex collection M. Pic, Muséum National d'Histoire Naturelle, Paris [type locality 'Bitlis' (Turkey)]

Provincial distribution in Turkey: TR-A: BT (Pic 1895a; Plavilstshikov 1958; Breuning 1962a; Löbl and Smetana 2010; Özdikmen 2010, 2012; Danilevsky 2015a)

Range: A: TR

*D. altinyaylaense Bernhauer and Peks, 2014a: 106

Type information: Holotype $\widehat{\sigma}$, collection Dieter Bernhauer, Bad Schwalbach [type locality 'W Altınyayla' (Turkey: Sivas)]

Provincial distribution in Turkey: TR-A: SV (Bernhauer and Peks 2014a; Danilevsky 2015a) Range: A: TR

*D. amanense Breuning, 1943: 94

Type information: Holotype $q$, ex collection Josef Breit > G. Frey, Naturhistorisches Museum Basel [type locality 'Amanos Mts.' (Turkey: Hatay)]

Provincial distribution in Turkey: TR-A: HT (Breuning 1943, 1962a; Löbl and Smetana 2010; Özdikmen 2010, 2012; Danilevsky 2015a)

Range: A: TR

*D. anatolicum Pic, 1900a: 12

Type information: Syntypes $\widehat{\partial}$ and $\not$, ex collection H. Tournier > M. Pic, Muséum National d'Histoire Naturelle, Paris [type locality 'Konya' (Turkey)] ininterruptum Pic, 1900a: 13 [HN] (D. anatolicum var.) [Turkey: Konya] mediointerruptum Pic, 1901: 73 (D. semilineatum var.) [Turkey: Konya] brevipenne Pic, 1902b: 10 [Turkey: Konya] 
chehirense Pic, 1902b: 10 (D. brevipenne var.) [Turkey: Konya] subuniforme Pic, 1902c: 27 [Turkey]

humeraliinterruptum Breuning, 1946: 108 (D. anatolicum m.) [Turkey: Konya] postapertum Breuning, 1946: 108 (D. anatolicum m.) [Turkey: Konya] pseudomerkli Breuning, 1946: 108 (D. anatolicum m.) [Turkey: Ankara] flavosignatum Breuning, 1946: 108 (D. anatolicum m.) [Turkey: Isparta]

Provincial distribution in Turkey: TR-A: AD AN ANT CA IP KA KN (Pic 1900a; Breuning 1946, 1962a; Demelt 1963; Braun 1978a; Adlbauer 1988; Önalp 1991; Özdikmen and Hasbenli 2004a; Özdikmen and Okutaner 2006; Löbl and Smetana 2010; Özdikmen 2010, 2012; Turgut and Özdikmen 2010; Okutaner et al. 2011; Küçükkaykı et al. 2013; Danilevsky 2015a)

Range: A: TR

*D. andirinense Bernhauer and Peks, 2011a: 218

Type information: Holotype $\hat{\sigma}$, collection Dieter Bernhauer, Bad Schwalbach [type locality 'Andırın (Altınyayla)' (Turkey: Kahramanmaraş)]

amaliae Pesarini and Sabbadini, 2013: 55 [Turkey: Kahramanmaraş: Andırın: Altınyayla] Provincial distribution in Turkey: TR-A: KA (Bernhauer and Peks 2011a, 2013b; Özdikmen 2015; Danilevsky 2015a)

Range: A: TR

*D. apicerufum Breuning, 1943: 91 [RN]

Type information: Holotype, ex collection J. Thomson > R. Oberthür, Muséum National d'Histoire Naturelle, Paris as D. sanguinolentum [type locality 'Armenia']

sanguinolentum J. Thomson, 1865: 548 [HN] [Armenia]

thomsonianum Plavilstshikov, 1948: 126 [RN] [Armenia]

lineiferum Breuning, 1946: 125 (D. apicerufum m.) [Armenia: Novo Bajaset]

Provincial distribution in Turkey: TR-A: EZ KAR TB (Plavilstshikov 1958; Breuning and Villiers 1967; Löbl and Smetana 2010; Özdikmen 2010; Danilevsky 2015a)

Range: $A: A B$ AR GG ?IN TR

*D. aracense Bernhauer and Peks, 2011b: 204

Type information: Holotype $\widehat{\partial}$, collection Dieter Bernhauer, Bad Schwalbach [type locality 'Araç' (Turkey: Kastamonu)]

Provincial distribution in Turkey: TR-A: KS (Bernhauer and Peks 2011b, 2013b; Danilevsky 2015a)

Range: A: TR

*D. arcivagum J. Thomson, 1867: 121

Type information: Holotype, ex collection J. Thomson > R. Oberthür, Muséum National d'Histoire Naturelle, Paris [type locality 'Gülek' (Turkey: İçel)]

cilicium Pic, 1904: 4 (D. arcivagum var.) [Turkey: Cilicia]

Provincial distribution in Turkey: TR-A: AD IC (Thomson 1867; Ganglbauer 1884; Pic 1904; Breuning 1962a; Löbl and Smetana 2010; Özdikmen 2010, 2012; Danilevsky 2015a) Range: A: TR 
*D. ardahense Breuning, 1975: 10

Type information: Holotype $q$, Muséum National d'Histoire Naturelle, Paris) [type locality 'Ardahan' (Turkey)]

Provincial distribution in Turkey: TR-A: AR (Breuning 1975; Löbl and Smetana 2010; Özdikmen 2010, 2012; Danilevsky 2015a)

Range: A: TR

D. atritarse Pic, 1931a: 10 (D. pedestre var.) status nov., comb. nov.

Type information: Syntypes $\hat{\sigma}$ and + , ex collection M. Pic, Muséum National d'Histoire Naturelle, Paris [type locality 'Greece']

Provincial distribution in Turkey: TR-A: CA; TR-E: ED (Adlbauer 1988; Althoff and Danilevsky 1997; Özdikmen and Demir 2006)

Range: E: GR TR A: TR

*D. avgadiense Bernhauer and Peks, 2011a: 215

Type information: Holotype $\widehat{\partial}$, collection Dieter Bernhauer, Bad Schwalbach [type locality 'Erdemli: Avgadı' (Turkey: İçel)]

Provincial distribution in Turkey: TR-A: IC (Bernhauer and Peks 2011a; Danilevsky 2015a) Range: A: TR

\section{D. axillare Küster, 1847a: 88}

D. axillare axillare Küster, 1847: 88

Type information: Holotype, ex collection Heinrich Carl Küster > Johann Menzel, Naturhistorisches Museum, Nürnberg [type locality 'Balkans' (Bulgaria)] kraatzi Tournier, 1872: 281 (D. pedestre var.) [Turkey] varnanum Pic, 1926: 13 (D. litigiosum var.) [Bulgaria: Varna]

Provincial distribution in Turkey: TR-E (Tournier 1872)

Range: E: BU TR

Other known subspecies: D. axillare moldavicum Dascalu and Fusu, 2012: $39 \mathrm{E}: \mathrm{RO}$

*D. bangi Heyden, 1894: 89

*D. bangi bangi Heyden, 1894: 89

Type information: Syntypes 4, ex collection L. von Heyden, Natur-Museum und Forschungs-Institut Senckenberg, Frankfurt am Main [type locality 'Kastamonu' (Turkey)] Provincial distribution in Turkey: TR-A: AN BO KR KS (Heyden 1894; Breuning 1946, 1962a; Demelt 1967; Sama 1982; Önalp 1991; Adlbauer 1992; Özdikmen et al. 2005; Özdikmen 2007, 2010, 2012; Löbl and Smetana 2010; Danilevsky 2015a) Range: A: TR

*D. bangi heinzorum Braun, 1975: 17 (D. heinzorum)

Type information: Holotype $\hat{\delta}$, collection Walter Braun, Tübingen [type locality 'İskilip' (Turkey: Çorum)]

Provincial distribution in Turkey: TR-A: CO KS (Braun 1975, 1978a, 1979; Adlbauer 1992; Löbl and Smetana 2010; Özdikmen 2010, 2012; Danilevsky 2015a)

Range: A: TR 
*D. bangi roridum Pesarini and Sabbadini, 1998: 55

Type information: Holotype $\hat{\jmath}$, collection Carlo Pesarini and Andrea Sabbadini, Milano [type locality 'Gerede' (Turkey: Bolu)]

Provincial distribution in Turkey: TR-A: BO (Pesarini and Sabbadini 1998; Löbl and Smetana 2010; Özdikmen 2010, 2011, 2012; Danilevsky 2015a)

Range: A: TR

*D. banjkovskyi Plavilstshikov, 1958: 147

Type information: Syntypes $4 \hat{\jmath} \hat{\partial}$ and $3 q \uparrow$, ex collection N. N. Plavilstshikov, Zoological Museum, University of Moscow [type locality 'Erzurum' (Turkey)]

Provincial distribution in Turkey: TR-A: EZ (Plavilstshikov 1958; Breuning 1962a; Löbl and Smetana 2010; Özdikmen 2010, 2012; Danilevsky 2015a)

Range: A: TR

\section{*D. basale Kraatz, 1873: 86}

Type information: Holotype $\delta$, ex collection Jacob Sturm, Zoologische Staatssammlung des Bayerischen Staates, München [type locality 'Armenien', but undoubtedly Turkey: ? Kars]

kagyzmanicum Suvorov, 1915: 120 [Turkey: Kars: Kağızman] Provincial distribution in Turkey: TR-A: KAR (Kraatz 1873; Suvorov 1915; Plavilstshikov 1958; Breuning 1962a; Löbl and Smetana 2010; Özdikmen 2012; Danilevsky 2015a) Range: A: TR

*D. beloni Pic, 1891: Ixxvii

Type information: Holotype $\widehat{\partial}$, ex collection M. Pic, Muséum National d'Histoire Naturelle, Paris [type locality 'Siberia?' but undoubtedly mislabeled, probably (Turkey: Hatay)]

Provincial distribution in Turkey: TR-A: HT (Pic 1891; Löbl and Smetana 2010; Özdikmen 2012; Danilevsky 2015a)

Range: A: TR

*D. bisignatum Jakovlev, 1899: 66

Type information: Lectotype $\hat{\partial}$, ex collection P. Semenov, Collection of Zoological Institute of the Russian Academy of Sciences, Saint-Petersbourg [type locality 'Artvin' (Turkey)]

Provincial distribution in Turkey: TR-A: ART (Jakovlev 1899; Plavilstshikov 1958; Breuning 1962a; Breuning and Villiers 1967; Löbl and Smetana 2010; Özdikmen 2010, 2012; Bernhauer and Peks 2013b; Danilevsky 2015a)

Range: A: TR

\section{D. bistriatum Pic, 1898: 55}

Type information: Holotype, ex collection Théry > M. Pic, Muséum National d'Histoire Naturelle, Paris) [type locality 'Caucasus']

Provincial distribution in Turkey: TR-A: IG (Breuning 1962a; Özdikmen 2010)

Range: $A$ : AB AR TR 
*D. bithyniense Chevrolat, 1856: 88

Type information: Holotype $\widehat{\partial}$, ex collection C. Bowring-Chevrolat, The Natural History Museum, London) [type locality 'Bursa' (Turkey)]

serotinum J. Thomson, 1865: 549 [Turkey: İzmir]

dorsoplagiatum Breuning, 1946: 120 (D. bithyniense m.) [Turkey: Bursa]

Provincial distribution in Turkey: TR-A: BL BS IS IZ KN KS SA (Chevrolat 1856; Thomson 1865; Ganglbauer 1884; Breuning 1946; 1962a; Demelt and Alkan 1962; Demelt 1963; Breuning and Villiers 1967; Adlbauer 1988; Önalp 1991; Özdikmen 2010; 2012; Löbl and Smetana 2010; Danilevsky 2015a)

Range: A: TR

D. blanchardi Mulsant and Rey, 1863: 147

Type information: Syntypes, Muséum National d'Histoire Naturelle, Paris [type locality 'Iran']

Provincial distribution in Turkey: TR-A: KA MA (Heyden 1888; Breuning 1962a; Löbl and Smetana 2010; Özdikmen 2010; Bernhauer and Peks 2011a; Danilevsky 2015a).

Range: A: IN TR

*D. blandulum Holzschuh, 1977: 131

Type information: Holotype $\widehat{\partial}$, collection Carolus Holzschuh, Villach [type locality 'Kuruca pass' (Turkey: Bingöl)]

Provincial distribution in Turkey: TR-A: BN (Holzschuh 1977; Löbl and Smetana 2010; Özdikmen 2010, 2012; Danilevsky 2012, 2015a)

Range: A: TR

*D. bodemeyeri K. Daniel, 1900: 140

Type information: Holotype $\hat{\jmath}$, ex collection K. Daniel, Deutsches Entomologisches Institut, Eberswalde [type locality 'Asia Minor (Lycaonia)' (Turkey: ?Konya)] rubricolle Breuning, 1946: 125 (D. bodemeyeri m.) [Turkey: Eskişehir]

Provincial distribution in Turkey: TR-A: AF AM AN BO ES IZ KN (K. Bodemeyer 1900; Daniel 1900; Breuning 1946, 1962a; Demelt 1963; Braun 1978a; Sama 1982; Önalp 1990; Löbl and Smetana 2010; Özdikmen 2010, 2011, 2012; Danilevsky 2015a)

Range: A: TR

*D. boluense Breuning, 1962b: 38

*D. boluense boluense Breuning, 1962b: 38

Type information: Holotype + , collection H. Muche [type locality 'Bolu Mts.' (Turkey: Bolu)]

nigrobasipenne Breuning, 1962b: 38 (D. boluense m.) [Turkey: Bolu]

Provincial distribution in Turkey: TR-A: BO (Breuning 1962b; Braun 1978a; Adlbauer 1992;

Pesarini and Sabbadini 1998; Löbl and Smetana 2010; Özdikmen 2010, 2011, 2012;

Danilevsky 2015a)

Range: A: TR 
*D. boluense corallinum Pesarini and Sabbadini, 1998: 53

Type information: Holotype $\hat{\sigma}$, collection Carlo Pesarini and Andrea Sabbadini, Milano [type locality 'Kızılcahamam: Çamlıdere' (Turkey: Ankara)]

Provincial distribution in Turkey: TR-A: AN (Braun 1978a; Sama 1982; Pesarini and Sabbadini 1998; Löbl and Smetana 2010; Özdikmen 2010, 2012; Danilevsky 2015a) Range: A: TR

*D. boluense imitator Pesarini and Sabbadini, 1998: 53

Type information: Holotype $\hat{\delta}$, collection Carlo Pesarini and Andrea Sabbadini, Milano [type locality 'Çubuk: Karagöl' (Turkey: Ankara)]

Provincial distribution in Turkey: TR-A: AN CN (Braun 1978a; Pesarini and Sabbadini 1998; Özdikmen 2006, 2010, 2012; Özdikmen and Demir 2006; Özdikmen et al. 2009; Löbl and Smetana 2010; Danilevsky 2015a)

Range: A: TR

\section{*D. boszdaghense Fairmaire, 1866: 275}

Type information: Syntypes, ex collection L. Fairmaire, Muséum National d'Histoire Naturelle, Paris [type locality 'Ovacık' (Turkey: İzmir)]

grammophilum J. Thomson, 1867: 70 ['Armenia', undoubtedly mislabeled] rufobasale Breuning, 1946: 107 (D. boszdaghense m.) [Turkey: İzmir: Bozdağ]

Provincial distribution in Turkey: TR-A: IZ (Fairmaire 1866; Thomson 1867; Ganglbauer 1884; Breuning 1946; 1962a; Özdikmen 2010; 2012; Löbl and Smetana 2010; Danilevsky 2015a) Range: A: TR

\section{*D. bouilloni Breuning and Ruspoli, 1975: 116}

Type information: Holotype $q$, ex collection M. Ruspoli, Museo Civico di Storia Naturale 'Giacomo Doria', Genova [type locality 'Mesudiye' (Turkey: Ordu)]

Provincial distribution in Turkey: TR-A: OR SM SV (Breuning and Ruspoli 1975; Löbl and Smetana 2010; Özdikmen 2010, 2012; Danilevsky 2015a)

Range: A: TR

*D. brauni Breuning, 1979: 92

Type information: Holotype $\hat{\delta}$, collection W. Heinz, Schwanfeld [type locality 'Tavas Valley' (Turkey: Denizli)]

Provincial distribution in Turkey: TR-A: DE (Breuning 1979; Löbl and Smetana 2010; Özdikmen 2010; Danilevsky 2015a)

Range: A: TR

*D. bremeri Breuning, 1981: 181

Type information: Holotype $\hat{\sigma}$, collection H. J. Bremer, Naturkundemuseum Erfurt [type locality 'Akyarma' (Turkey: Afyonkarahisar)]

Provincial distribution in Turkey: TR-A: AF (Breuning 1981; Löbl and Smetana 2010; Özdikmen 2010, 2012; Danilevsky 2015a)

Range: A: TR 


\section{D. breuningi Heyrovský, 1943: 78}

Type information: Syntypes $\widehat{\jmath} \widehat{\partial}$ and $q q$, ex collection Leo Heyrovský, Musée National de Prague [type locality 'Thrazien: Umgebung von Xanthi, Ferre und Badoma' (Greece)] Provincial distribution in Turkey: TR-E (Althoff and Danilevsky 1997; Löbl and Smetana 2010; Özdikmen 2010; Danilevsky 2015a)

Range: E: GR TR

*D. bulgharmaadense Breuning, 1946: 116

Type information: Holotype $q$, ex collection S. Breuning, Muséum d'Histoire Naturelle de Genève [type locality 'Bulghar-Maaden [Bolkar Mts.-Maden vill. (Çamardı)]' (Turkey: Niğde)]

Provincial distribution in Turkey: TR-A: NI (Breuning 1946, 1962a; Löbl and Smetana 2010; Özdikmen 2010, 2012; Danilevsky 2015a)

Range: A: TR

*D. cachinno J. Thomson, 1867: 120

Type information: Syntypes, ex collection J. Thomson > R. Oberthür, Muséum National d'Histoire Naturelle, Paris [type locality 'Caramania' (Turkey: Karaman)] immersum Tournier, 1872: 281 [Turkey] fuscoflavum Breuning, 1946: 110 (D. cachinno m.) [Turkey]

Provincial distribution in Turkey: TR-A: KM US (Thomson 1867; Breuning 1946, 1962a; Demelt 1963; Löbl and Smetana 2010; Özdikmen 2010, 2012; Danilevsky 2015a) Range: A: TR

*D. caglayanceritense Bernhauer and Peks, 2013a: 314

Type information: Holotype $\widehat{\partial}$, collection Dieter Bernhauer, Bad Schwalbach [type locality 'Çağlayancerit' (Turkey: Kahramanmaraş)]

Provincial distribution in Turkey: TR-A: KA (Bernhauer and Peks 2013a; Danilevsky 2015a) Range: A: TR

\section{*D. carinipenne Pic, 1900a: 13}

Type information: Holotype, ex collection M. Pic, Muséum National d'Histoire Naturelle, Paris [type locality 'Akbez' (Turkey: Hatay)] quinquevittatum Breuning, 1946: 127 (D. carinipenne m.) [Turkey: Gaziantep]

Provincial distribution in Turkey: TR-A: GA HT (Pic 1900a; Breuning 1946, 1962a; Löbl and Smetana 2010; Özdikmen 2010, 2012; Danilevsky 2015a)

Range: A: TR

\section{*D. carolisturanii Breuning and Ruspoli, 1971: 128}

Type information: Holotype $\hat{\sigma}$, ex collection S. Breuning, Muséum d'Histoire Naturelle de Genève [type locality 'Kümbet' (Turkey: Giresun)]

baiburtense Breuning, 1975: 10 (D. carolisturanii m.) [Turkey: Bayburt]

Provincial distribution in Turkey: TR-A: BY GI OR (Breuning and Ruspoli 1971; Breuning 1975; Braun 1978a; Sama 1982; Löbl and Smetana 2010; Özdikmen 2010, 2012; Danilevsky 2015a)

Range: A: TR 
D. catenatum Waltl, 1838: 469

D. catenatum catenatum Waltl, 1838: 469

Type information: Holotype ${ }^{\prime}$, ex collection Imre Frivaldszky, Magyar Természettudományi Mûzeum, Budapest [type locality 'Turkei' (Turkey)]

divisum Germar, 1839: 15 [Turkey]

mancum Gistel, 1848: 431 [?Syria]

subobliteratum T. Pic, 1899: 351 (D. divisum var.) [Turkey]

uninterruptum T. Pic, 1899: 351 (D. divisum var.) [Turkey: İzmir]

dorsale Pic, 1907: 179 (D. divisum var.) [Turkey: İzmir]

sparsedivisum Pic, 1911: 185 (D. divisum var.) [Greece: Lesbos (Mytilene) Island]

smyrnense Pic, 1917a: 10 (D. divisum var.) [Turkey: İzmir]

thebesianum Pic, 1942d: 1 (D. divisum var.) [Greece: Thebes]

bonyi Pic, 1942a: 1 (D. divisum var.) [?Syria]

smyrnanum Breuning, 1946: 106 [RN] [Turkey: İzmir]

postmediomaculatum Breuning, 1946: 106 (D. divisum m.) [Turkey: İzmir]

chioticum Breuning, 1946: 106 (D. divisum m.) [Greece: Chios Island]

tenebripes Breuning, 1946: 106 (D. divisum m.) [Greece: Samos Island]

parteinterruptum Breuning, 1962a: 384 (D. divisum m.) [Turkey: Manisa: Demirci]

subatrum Breuning, 1962a: 385 (D. divisum m.) [Turkey: Balıkesir]

peratrum Breuning, 1962a: 385 (D. divisum m.) [Greece: Lesbos Island: Ayassos]

submytilinense Breuning, 1962a: 386 (D. divisum m.) [Greece: Lesbos Island]

dorsaloides Breuning, 1966a: 20 (D. divisum m.) [Turkey: İzmir: Bozdağ]

Provincial distribution in Turkey: TR-A: BL BS BU ES IP IZ KN KU MN (Küster 1846a; Kraatz 1873; Ganglbauer 1884; Pic 1899, 1917a; Breuning 1946; 1962a, 1966a; Demelt 1963; Sama 1982; Önalp 1991; Özdikmen 2010; 2012; Löbl and Smetana 2010; Şenyüz and Özdikmen 2013; Danilevsky 2015a)

Range: E: GR A: TR

*D. catenatum dissimile Ganglbauer, 1884: 458 (D. divisum var.)

Type information: Syntypes, ex collection L. Ganglbauer, Naturhistorisches Museum Wien [type locality 'European Turkey']

Provincial distribution in Turkey: TR-E: CA ED (Ganglbauer 1884; Pesarini and Sabbadini 2009; Löbl and Smetana 2010; Özdikmen 2010, 2012; Sama et al. 2010; Danilevsky 2015a) Range: E: TR

\section{*D. catenatum intercisum Kraatz, 1873: 66 (D. latevittatum var.)}

Type information: Holotype, ex Musaeo Mniszech > R. Oberthür, Muséum National d'Histoire Naturelle, Paris [type locality 'Kleinasien' (Turkey: Diyarbakır)]

Provincial distribution in Turkey: TR-A: AD ADY IC MR NI OS SV (Kraatz 1873; Bodemeyer 1900; Braun 1978a; Önalp 1990; Özdikmen 2006, 2010, 2012; Löbl and Smetana 2010; Danilevsky 2015a)

Range: A: TR

*D. catenatum loratum J. Thomson, 1867: 123 (D. loratum)

Type information: Holotype $\hat{\sigma}$, ex collection J. Thomson > R. Oberthür, Muséum National d'Histoire Naturelle, Paris [type locality 'Smyrne' (Turkey: İzmir)] 
Provincial distribution in Turkey: TR-A: CA IZ (Thomson 1867; Braun 1978a; Löbl and Smetana 2010; Özdikmen 2010, 2012; Danilevsky 2015a)

Range: A: TR

\section{D. catenatum mytilenense Kraatz, 1873: 66 (D. latevittatum var.)}

Type information: Holotype, ex Musaeo Mniszech > R. Oberthür, Muséum National d'Histoire Naturelle, Paris [type locality 'Kleinasien' (Turkey)]

latevittatum Kraatz, 1873: 66 [Turkey]

Provincial distribution in Turkey: TR-A: IZ (Kraatz 1873; Löbl and Smetana 2010; Özdikmen 2010)

Range: E: GR A: TR

*D. catenatum oedemischense Heyrovský, 1932: 104 (D. divisum var.)

Type information: Syntypes 5, Zoologische Staatssammlung des Bayerischen Staates, München [type locality 'Ödemiş' (Turkey: İzmir)]

Provincial distribution in Turkey: TR-A: IZ (Heyrovský 1932; Löbl and Smetana 2010; Özdikmen 2010, 2012; Danilevsky 2015a)

Range: A: TR

*D. catenatum subdivisum Breuning, 1955: 263 (D. divisum ssp.)

Type information: Holotype $\widehat{\partial}$, Magyar Természettudományi Mûzeum, Budapest) [type locality 'Ankara' (Turkey)]

submediomaculatum Breuning, 1955: 263 (D. divisum subdivisum m.) [Turkey: Ankara] Provincial distribution in Turkey: TR-A: AN (Breuning 1955; Fuchs and Breuning 1971; Özdikmen 2006, 2010, 2012; Löbl and Smetana 2010; Danilevsky 2015a)

Range: A: TR

Other known subspecies: D. catenatum bleusei Pic, 1899: $300 \mathrm{E}: \mathrm{GR}$

*D. cinctellum Fairmaire, 1866: 272

Type information: Syntypes $\hat{\partial} \hat{\partial}$, ex collection L. Fairmaire, Muséum National d'Histoire Naturelle, Paris [type locality 'Kisilgye-Aole (Ödemiş: Kızılca köyü)' (Turkey: İzmir)] aureofasciatum Breuning, 1947: 168 ['Kisilgye-Aole', Turkey: İzmir: Ödemiş: Kızılca] quadrifuscoplagiatum Breuning, 1947: 168 (D. cinctellum m.) [Turkey: İzmir: Ödemiş] reductum Breuning, 1947: 168 [Turkey: İzmir: Ödemiş]

Provincial distribution in Turkey: TR-A: HT IZ KN (Fairmaire 1866; Ganglbauer 1884; Pic 1917a; Breuning 1947, 1962a; Adlbauer 1992; Löbl and Smetana 2010; Özdikmen 2010, 2012; Danilevsky 2015a)

Range: A: TR

\section{D. cingulatum Ganglbauer, 1884: 484}

Type information: Syntypes $2 q q$, ex collection L. Ganglbauer, Naturhistorisches Museum Wien [type locality 'Persien' (Iran)]

invicinum Pic, 1902a: 93 [Turkey: Diyarbakır]

persianum Breuning, 1943: 94 [Iran]

Provincial distribution in Turkey: TR-A: DI (Pic 1902a; Breuning 1962a; Villiers 1967; Löbl and Smetana 2010; Özdikmen 2010; Danilevsky 2015a) 
Range: A: IN TR

*D. coiffaiti Breuning, 1962a: 392

Type information: Holotype $\widehat{\partial}$, ex collection Coiffait, Muséum National d'Histoire Naturelle, Paris [type locality 'Manyas' (Turkey: Balıkesir)]

Provincial distribution in Turkey: TR-A: BL (Breuning 1962a; Pesarini and Sabbadini 2009; Löbl and Smetana 2010; Özdikmen 2010, 2012; Danilevsky 2015a)

Range: A: TR

D. condensatum Küster, 1852: 92

Type information: Syntypes $\hat{\partial} \hat{\partial}$ and $\phi++$, ex collection Kiesenwetter, Zoologische Staatssammlung des Bayerischen Staates, München [type locality 'Turkei' (Turkey)] punctatissimum Ganglbauer, 1884: 94 (D. condensatum var.) [Turkey] adampolum Pic, 1917b: 6 (D. condensatum var.) [Turkey: İstanbul: Polonezköy (Adampol)]

inlineatum Pic, 1917b: 6 (D. condensatum var.) [Turkey]

Provincial distribution in Turkey: TR-A: IS KO TR-E: IS (Ganglbauer 1884; Bodemeyer 1906; Pic 1917b; Demelt and Alkan 1962; Breuning 1962a; Demelt 1963; Braun 1978a; Önalp 1990; Adlbauer 1992; Althoff and Danilevsky 1997; Löbl and Smetana 2010; Özdikmen 2010, 2012; Danilevsky 2015a)

Range: E: BU TR A: TR

*D. confluens Fairmaire, 1866: 274

Type information: Syntypes $\hat{\delta} \hat{\sigma}$ and $q q$, ex collection L. Fairmaire, Muséum National d'Histoire Naturelle, Paris [type locality 'Bozdağ' (Turkey: İzmir)]

subobscuripes Pic, 1914: 9 [Turkey: İzmir: Bozdağ]

Provincial distribution in Turkey: TR-A: IZ (Fairmaire 1866; Pic 1914; Pesarini and Sabbadini 1998; Löbl and Smetana 2010; Özdikmen 2010, 2012; Danilevsky 2015a) Range: A: TR

*D. conspersum Pesarini and Sabbadini, 2013: 56

Type information: Holotype $\hat{\jmath}$, collection Carlo Pesarini and Andrea Sabbadini, Milano [type locality 'Bayramgazi' (Turkey: Afyonkarahisar)]

Provincial distribution in Turkey: TR-A: AF (Pesarini and Sabbadini 2013; Danilevsky 2015a)

Range: A: TR

\section{D. cretosum 1864: 479}

Type information: Syntypes $\hat{\partial} \widehat{\partial}$ and $q \propto+$, Naturhistorisches Museum Wien [type locality 'Achalzik' (Georgia)]

niveisparsum J. Thomson, 1865: 548 [Caucasus]

sobrinum Pic, 1898: 55 (D. niveisparsum var.) [Caucasus]

postobliteratum Pic, 1942c: 1 (D. niveisparsum var.) [Caucasus]

Provincial distribution in Turkey: TR-A: EZ MN (Plavilstshikov 1958; Sama 1982; Önalp 1990; Löbl and Smetana 2010; Özdikmen 2010; Danilevsky 2015a)

Range: A: GG TR 
Type information: Holotype, ex Musaeo Schönherr, Naturhistoriska Riksmuseet, Stockholm [type locality unknown but very likely İzmir (Turkey)]

smyrnensis Linnaeus, 1757: 411 (Cerambyx) [Turkey: İmir]

graecum Waltl, 1838: 470 [Greece]

cruciator Gistel, 1848: 131 [?Syria]

bifidum Chevrolat, 1882: 60 [Turkey: İzmir]

lunulatum Pic, 1900a: 12 (D. graecum var.) [Turkey: İzmir]

integrofasciatum K. Daniel, 1900: 140 (D. crux var.) [Turkey: İzmir: Bozdağ]

niveopictum K. Daniel, 1900: 140 (D. crux var.) [Turkey: İzmir: Bozdağ]

biinterruptum Pic, 1901: 19 (D. crux var.) [Turkey]

grandevittatum Pic, 1904: 7 [Turkey: Imbey]

subfurcatum Pic, 1914: 9 (D. cinctellum var.) [Turkey]

brevedisjunctum Pic, 1929: 9 (D. crux var.) [Turkey]

lateargenteum Pic, 1929: 9 (D. crux var.) [Turkey]

quadriinterruptum Breuning, 1946: 101 (D. smyrnense m.) [Turkey: İzmir]

subniveopictum Breuning, 1946: 101 (D. smyrnense m.) [Turkey: Eskişehir]

holodisjunctum Breuning, 1946: 101 (D. smyrnense m.) [Turkey: Eskişehir]

rufulum Breuning, 1946: 106 (D. smyrnense m.) [Turkey: İzmir]

dorsoabbreviatum Breuning, 1946: 106 (D. smyrnense m.) [Turkey: Konya: Akşehir]

semiinterruptum Breuning, 1946: 106 (D. smyrnense m.) [Turkey: Eskişehir]

lunulatoides Breuning, 1948: 58 (D. smyrnense m.) [Turkey]

bodemeyerianum Breuning, 1962a: 279 (D. smyrnense m.) [Turkey: İzmir: Bozdağ]

Provincial distribution in Turkey: TR-A: BI BL BS ES IS IZ KN KU US (Ganglbauer 1884; Bodemeyer 1900, 1906; Pic 1900a; Breuning 1946, 1962a; Demelt 1963; Tuatay et al. 1972; Braun 1978a; Adlbauer 1988; Önalp 1991; Özdikmen et al. 2005; Löbl and Smetana 2010; Özdikmen 2010, 2012; Danilevsky 2015a)

Range: A: TR

*D. culminicola J. Thomson, 1867: 122

Type information: Holotype $\hat{\sigma}$, ex collection J. Thomson > R. Oberthür, Muséum National d'Histoire Naturelle, Paris [type locality 'Caramania' (Turkey: Karaman)]

Provincial distribution in Turkey: TR-A: KM (Thomson 1867; Ganglbauer 1884; Breuning 1962a; Löbl and Smetana 2010; Özdikmen 2010, 2012; Danilevsky 2015a)

Range: A: TR

*D. delagrangei Pic, 1894: 110

Type information: Syntypes $\hat{\sigma}$ and + , ex collection M. Pic, Muséum National d'Histoire Naturelle, Paris [type locality 'Akbez' (Turkey: Hatay)]

mardinense Pic, 1900b: 6 (D. accola var.) [Turkey: Mardin]

fuscopubescens Pic, 1900a: 12 (D. delagrangei var.) [Turkey: Hatay: Akbez]

Provincial distribution in Turkey: TR-A: GA HT KL MR OS (Pic 1894, 1897, 1900a, 1900b; Breuning 1962a; Braun 1978a; Löbl and Smetana 2010; Özdikmen 2010, 2012; Bernhauer and Peks 2011a; Danilevsky 2015a)

Range: A: TR 
*D. deyrollei Ganglbauer, 1884: 482

*D. deyrollei deyrollei Ganglbauer, 1884: 482

Type information: Syntypes $\widehat{\jmath}$, ex collection L. Ganglbauer, Naturhistorisches Museum Wien [type locality 'Bitlis' (Turkey)]

subalbipenne Breuning, 1946: 116 (D. deyrollei m.) [Turkey: Van]

Provincial distribution in Turkey: TR-A: BT VA (Ganglbauer 1884; Breuning 1946, 1962a;

Plavilstshikov 1958; Fuchs and Breuning 1971; Holzschuh 1980; Önalp 1990; Löbl and Smetana 2010; Özdikmen 2010, 2012; Danilevsky 2015a)

Range: A: TR

*D. deyrollei opacum Pesarini and Sabbadini, 2013: 54

Type information: Holotype $\hat{\sigma}$, collection Carlo Pesarini and Andrea Sabbadini, Milano [type locality 'Ahır Mt.' (Turkey: Kahramanmaraş)]

melas Breuning, 1946: 116 (D. deyrollei m.) [Turkey: Kahramanmaraş]

Provincial distribution in Turkey: TR-A: GA KA (Breuning 1946, 1962a; Pesarini and Sabbadini 2013; Danilevsky 2015a)

Range: A: TR

D. dimidiatum Motschulsky, 1838: 186

D. dimidiatum dimidiatum Motschulsky, 1838: 186

Type information: Syntypes $\hat{\partial} \sigma^{\lambda}$ and $q \propto$, ex collection V. I. Motschulsky, Zoological Museum, University of Moscow [type locality 'Armenia']

erivanicum Pic, 1901: 91 [Armenia: Erevan]

reductemaculatum Breuning, 1946: 123 (D. dimidiatum m.) [Transcaucasia]

flavescens Breuning, 1946: 124 (D. dimidiatum m.) [Armenia: Erivan]

nigrovittatum Breuning, 1946: 124 (D. dimidiatum m.) [Armenia: Erivan]

nigroreductum Breuning, 1946: 124 (D. dimidiatum m.) [Armenia: Erivan]

unimaculatum Breuning, 1946: 124 (D. dimidiatum m.) [Armenia: Erivan]

degaullei Pic, 1948: 13 (D. dimidiatum var.)

transitorium Pic, 1948: 13

Provincial distribution in Turkey: TR-A: AG ER EZ MA (Thomson 1867; Plavilstshikov 1958; Villiers 1967; Gfeller 1972; Özbek 1978; Braun 1978a; Önalp 1991; Özdikmen and Hasbenli 2004b; Löbl and Smetana 2010; Özdikmen 2010; Danilevsky 2015a)

Range: $A$ : $A B$ AR GG IN TR

*D. dimidiatum kelkiticum Özdikmen and Hasbenli, 2004b: 23

Type information: Holotype $\hat{\jmath}$, Zoological Museum of Gazi University, Ankara [type locality 'Kelkit' (Turkey: Gümüşhane)]

Provincial distribution in Turkey: TR-A: GU (Özdikmen and Hasbenli 2004b; Özdikmen 2010, 2012; Danilevsky 2015a)

Range: A: TR

*D. dimidiatum korgei Breuning, 1966a: 21

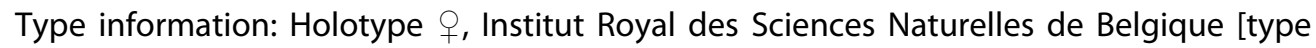
locality 'near Pülümür' (Turkey: Tunceli)] 
Provincial distribution in Turkey: TR-A: TU (Breuning 1966a; Löbl and Smetana 2010; Özdikmen 2010, 2012; Danilevsky 2015a)

Range: A: TR

\section{*D. dobrovljanskii Suvorov, 1915: 116}

Type information: Holotype $\hat{\sigma}$, ex collection G. Suvorov, Zoological Museum, University of Moscow [type locality 'Erivansk. g.' (should be 'NE Turkey')]

Provincial distribution in Turkey: TR-A: North-Eastern TR (Suvorov 1915; Löbl and Smetana 2010; Özdikmen 2010, 2012; Danilevsky 2015a)

Range: A: TR

*D. drusoides Breuning, 1962a: 375

Type information: Holotype $\widehat{\partial}$, ex collection Lepesme, Musée d'Histoire Naturelle de Lyon [type locality 'Amanos Mts.' (Turkey: Hatay)]

Provincial distribution in Turkey: TR-A: HT (Breuning 1962a; Löbl and Smetana 2010; Özdikmen 2010, 2012; Danilevsky 2015a)

Range: A: TR

\section{*D. eflaniense Bernhauer and Peks, 2011b: 205}

Type information: Holotype $\hat{\sigma}$, collection Dieter Bernhauer, Bad Schwalbach [type locality 'Eflani: Kırıklar' (Turkey: Karabük)]

Provincial distribution in Turkey: TR-A: KR KS (Bernhauer and Peks 2011b; Danilevsky 2015a)

Range: A: TR

*D. elazigi Fuchs and Breuning, 1971: 439

Type information: Holotype $\delta$, Anatolienexpedition 1966, Naturhistorisches Museum Wien [type locality 'Buğlan pass' (Turkey: Muş)]

holzschuhi Breuning, 1974a: 148 [Turkey: Muş: Buğlan pass]

Provincial distribution in Turkey: TR-A: BN EL MU (Fuchs and Breuning 1971; Breuning 1974a; Holzschuh 1980; Löbl and Smetana 2010; Özdikmen 2010, 2012; Danilevsky 2015a) Range: A: TR

*D. enricisturanii Breuning and Ruspoli, 1971: 127

*D. enricisturanii densepunctatum Braun, 1978a: 107

Type information: Holotype $\hat{\jmath}$, collection Walter Braun, Tübingen [type locality 'Vezirköprü' (Turkey: Samsun)]

Provincial distribution in Turkey: TR-A: SM (Braun 1978a; Löbl and Smetana 2010; Özdikmen 2010, 2012; Danilevsky 2015a)

Range: A: TR

*D. enricisturanii enricisturanii Breuning and Ruspoli, 1971: 127

Type information: Holotype $\hat{\jmath}$, ex collection S. Breuning, Muséum d'Histoire Naturelle de Genève [type locality 'SE Tosya' (Turkey: Kastamonu)]

Provincial distribution in Turkey: TR-A: CO KS (Breuning and Ruspoli 1971; Braun 1975, 1978a; Adlbauer 1992; Löbl and Smetana 2010; Özdikmen 2010, 2012; Danilevsky 2015a) Range: A: TR 


\section{D. equestre Laxmann, 1770: 596 (Cerambyx)}

*D. equestre bernhauerorum Peks, 2010: 213 status nov., comb. nov.

Type information: Holotype $\hat{\sigma}$, collection Heinz Peks, Schwalbach [type locality 'İznik: Hacıosman village' (Turkey: Bursa)]

Provincial distribution in Turkey: TR-A: BS (Peks 2010; Özdikmen 2012; Danilevsky 2015a) Range: A: TR

\section{D. equestre bisuturale Jureček, 1933: 128 (D. equestre var.)}

Type information: Syntypes $7 \hat{\jmath} \widehat{\jmath}$ and + , ex collection Štepán Jurecek, Musée National de Prague [type locality 'Griechenland, Bos-Dagh-Gebirge bei Drama' (Greece)]

Provincial distribution in Turkey: TR-E (Breuning 1962a; Althoff and Danilevsky 1997; Danilevsky 2015a)

Range: E: BU GR TR

*D. equestre festivum Pesarini and Sabbadini, 2013: 58

Type information: Holotype $\hat{\jmath}$, collection Carlo Pesarini and Andrea Sabbadini, Milano [type locality 'Sündiken Mts.: Karatepe' (Turkey: Eskişehir)]

Provincial distribution in Turkey: TR-A: ES (Pesarini and Sabbadini 2013; Danilevsky 2015a)

Range: A: TR

*D. equestre nogelli Fairmaire, 1866: 270 (D. nogelli)

Type information: Syntypes $\hat{\delta} \hat{\delta}$ and $q \circ$, ex collection L. Fairmaire, Muséum National d'Histoire Naturelle, Paris [type locality 'Bozdağ et Ovacık' (Turkey: İzmir)]

nogelii Thomson, 1867: 58 [?Armenia]

exclamationis Thomson, 1867: 58 (D. nogelii var.) [?Armenia]

Provincial distribution in Turkey: TR-A: IZ EZ (Fairmaire 1866; Ganglbauer 1884; Breuning 1962a; Önalp 1991; Pesarini and Sabbadini 1998, 2013, Löbl and Smetana 2010; Özdikmen 2010, 2012; Peks 2010; Danilevsky 2015a)

Range: A: TR

Other known subspecies: D. equestre equestre Laxmann, 1770: 596 (Cerambyx equestris) E: CT ST UK A: GG; D. equestre reclinatum Kraatz, 1892: 173 E: AL GR MC YU; D. equestre transsilvanicum Ganglbauer, 1884: $462 \mathrm{E}:$ MD RO

*D. erdemi Özdikmen, Kaya and Al-Hamadani, 2014: 179

Type information: Holotype $\widehat{\partial}$, collection H. Özdikmen, Zoological Museum of Gazi University, Ankara [type locality 'Sungurlu road' (Turkey: Çorum)]

Provincial distribution in Turkey: TR-A: CO (Özdikmen et al. 2014; Danilevsky 2015a)

Range: A: TR

*D. eskipazarense Bernhauer and Peks, 2011 b: 203

Type information: Holotype $\widehat{\partial}$, collection Dieter Bernhauer, Bad Schwalbach [type locality 'Eskipazar' (Turkey: Karabük)]

Provincial distribution in Turkey: TR-A: KR (Bernhauer and Peks 2011 b; Danilevsky 2015a) Range: A: TR 


\section{D. ferruginipes Ménétriés, 1836: 151}

Type information: Holotype, Collection of Zoological Institute of the Russian Academy of Sciences, Saint-Petersbourg [type locality 'İstanbul' (Turkey)]

thracicum Küster, 1846a: 90 [Turkey: İstanbul]

sublineatum Pic, 1909b: 99 (D. ferruginipes var.) [Turkey]

subfuscopubens Pic, 1911: 7 (D. ferruginipes var.) [Turkey: İstanbul: Alem Mt.]

Provincial distribution in Turkey: TR-A: IS KO SA; TR-E: IS (Ménétriés 1836; Küster 1846a; Ganglbauer 1884; Breuning 1962a; Braun 1978a; Önalp 1991; Althoff and Danilevsky 1997; Löbl and Smetana 2010; Özdikmen 2010, 2012; Danilevsky 2015a)

Range: E: GR TR A: TR

*D. formosum Kraatz, 1871: 411

*D. formosum formosum Kraatz, 1871: 411

Type information: Syntypes, ex collection G. Kraatz, Deutsches Entomologisches Institut, Eberswalde [type locality 'Caucasus' but undoubtedly mislabeled, should be Turkey] inincisum Breuning, 1946: 124 (D. formosum m.) ['Ain-Tab', Turkey: Gaziantep]

Provincial distribution in Turkey: TR-A: GA (Kraatz 1871; Breuning 1946, 1962a; Löbl and Smetana 2010; Özdikmen 2010, 2012; Danilevsky 2015a)

Range: A: TR

\section{*D. formosum ponticum Breuning, 1964a: 1}

Type information: Holotype $\widehat{\partial}$, ex collection S. Breuning, Muséum d'Histoire Naturelle de Genève [type locality 'Eğribel pass' (Turkey: Giresun)]

Provincial distribution in Turkey: TR-A: GI TO (Breuning 1962a, 1964a; Braun 1978a; Löbl and Smetana 2010; Özdikmen 2010, 2012; Danilevsky 2015a)

Range: A: TR

\section{D. gallipolitanum J. Thomson, 1867: 59}

\section{D. gallipolitanum gallipolitanum J. Thomson, 1867: 59}

Type information: Holotype $\hat{\sigma}$, ex collection J. Thomson > R. Oberthür, Muséum National d'Histoire Naturelle, Paris [type locality 'Gallipoli' (Turkey: Çanakkale)] suturaalba Desbrochers des Loges, 1870: 125 [Turkey: Çanakkale] gandolphei Tournier, 1872: 288 [Turkey: Çanakkale] potamense Jurecek, 1931: 124 (D. gallipolitanum ab.) [Turkey: Sinop: Dedeağaç env.] biapicevittatum Breuning, 1946: 110 (D. gallipolitanum m.) [Turkey: İstanbul] latesuturale Breuning, 1946: 110 (D. gallipolitanum m.) [Greece: Thrace] subregulare Breuning, 1948: 59 (D. gallipolitanum m.) [Turkey: Çanakkale (European Turkey part)] stramentosopubescens Breuning, 1948: 59 (D. gallipolitanum m.) ['Turkey: Port Baclar',

Turkey: ?ंstanbul: ?Bağcılar]

plurivittatum Breuning, 1948: 59 (D. gallipolitanum m.) ['Turkey: Port Baclar', Turkey: ? İstanbul: ?Bağcılar]

Provincial distribution in Turkey: TR-A: CA IS SN; TR-E: CA ED IS (Thomson 1867; Ganglbauer 1884; Breuning 1946, 1948, 1962a; Braun 1978a; Adlbauer 1988; Önalp 1990; Althoff and Danilevsky 1997; Löbl and Smetana 2010; Özdikmen 2010, 2012; Danilevsky 2015a) 
Range: E: GR TR A: TR

Other known subspecies: D. gallipolitanum fumidum Pesarini and Sabbadini, 2010: $186 \mathrm{E}$ :

GR; D. gallipolitanum samothrakeanum Breuning, 1962a: $326 \mathrm{E}: \mathrm{GR}$

*D. gebzeense Breuning, 1974a: 148

Type information: Holotype $\hat{\sigma}$, Collection Carolus Holzschuh, Villach [type locality 'Gebze' (Turkey: Kocaeli)]

tosyense Breuning in Sama, 1982: 223 [Turkey: Kastamonu: Tosya]

Provincial distribution in Turkey: TR-A: KO KS (Breuning 1974a; Braun 1975, 1978a; Sama 1982; Özdikmen 2010, 2012; Özdikmen and Kaya 2015e)

Range: A: TR

*D. gencense Bernhauer and Peks, 2014a: 110

Type information: Holotype $\hat{\jmath}$, collection Dieter Bernhauer, Bad Schwalbach) [type locality 'SW Genç' (Turkey: Bingöl)]

Provincial distribution in Turkey: TR-A: BN (Bernhauer and Peks 2014a; Danilevsky 2015a) Range: A: TR

*D. glabricolle Breuning, 1943: 92

Type information: Holotype + , ex collection S. Breuning, Muséum d'Histoire Naturelle de Genève [type locality 'Anatolia' (Turkey)]

Provincial distribution in Turkey: TR-A (Breuning 1943, 1962a; Löbl and Smetana 2010; Özdikmen 2010, 2012; Danilevsky 2015a)

Range: A: TR

*D. goelbasiense Bernhauer and Peks, 2013a: 310

*D. goelbasiense goelbasiense Bernhauer and Peks, 2013a: 310

Type information: Holotype $\hat{\sigma}$, collection Dieter Bernhauer, Bad Schwalbach [type locality 'Gölbaşı' (Turkey: Adıyaman)]

Provincial distribution in Turkey: TR-A: ADY (Bernhauer and Peks 2013a; Danilevsky 2015a)

Range: A: TR

*D. goelbasiense karadutense Bernhauer and Peks, 2013a: 311

Type information: Holotype $\widehat{\partial}$, collection Dieter Bernhauer, Bad Schwalbach [type locality 'Kahta: Karadut' (Turkey: Adıyaman)]

Provincial distribution in Turkey: TR-A: ADY (Bernhauer and Peks 2013a; Danilevsky 2015a)

Range: A: TR

*D. goelbasiense kathaense Bernhauer and Peks, 2013a: 312

Type information: Holotype $\hat{\sigma}$, collection Dieter Bernhauer, Bad Schwalbach [type locality 'Kahta' (Turkey: Adıyaman)]

Provincial distribution in Turkey: TR-A: ADY (Bernhauer and Peks 2013a; Danilevsky 2015a)

Range: A: TR 
*D. goelbasiense oeksuezdagense Bernhauer and Peks, 2013a: 311

Type information: Holotype $\widehat{\jmath}$, collection Dieter Bernhauer, Bad Schwalbach [type locality 'Öksüz Mt.' (Turkey: Kahramanmaraş)]

Provincial distribution in Turkey: TR-A: KA (Bernhauer and Peks 2013a; Danilevsky 2015a) Range: A: TR

*D. guzeldereense Bernhauer and Peks, 2012: 213

Type information: Holotype $\widehat{\partial}$, collection Dieter Bernhauer, Bad Schwalbach [type locality 'Başkale: Güzeldere pass' (Turkey: Van)]

guezeldereense Bernhauer and Peks, 2013b: 336 [unjustified emendation]

Provincial distribution in Turkey: TR-A: VA (Bernhauer and Peks 2012, 2013b; Danilevsky 2015a)

Range: A: TR

*D. hacgedigiense Bernhauer and Peks, 2011a: 216

Type information: Holotype $\widehat{\partial}$, collection Dieter Bernhauer, Bad Schwalbach [type locality 'Haçgediği' (Turkey: İçel)]

Provincial distribution in Turkey: TR-A: IC (Bernhauer and Peks 2011a, 2013b; Danilevsky 2015a)

Range: A: TR

\section{D. haemorrhoidale Hampe, 1852: 313}

Type information: Syntypes, ex collection Moritz Wagner, Museum für Naturkunde der Humboldt-Universität zu Berlin [type locality 'Armenia']

sublineiferum Breuning, 1946: 125 (D. haemorrhoidale m.) [Armenia: Erivan]

vittidorsum Plavilstshikov, 1958: 113 (D. haemorrhoidale m.) [?Armenia]

Provincial distribution in Turkey: TR-A: AG AN EZ (Plavilstshikov 1958; Gfeller 1972; Braun 1978a; Önalp 1990; Löbl and Smetana 2010; Özdikmen 2010; Danilevsky 2015a)

Range: A: AR IN TR

D. halepense Kraatz, 1873: 72 (D. saulcyi var.)

D. halepense halepense Kraatz, 1873: 72 (D. saulcyi var.)

Type information: Syntypes, ex collection G. Kraatz, Deutsches Entomologisches Institut, Eberswalde [type locality 'Aleppo' (Syria)]

internesignatum Pic, 1914: 79 (D. aleppense var.) [Syria: Aleppo]

latealbum Pic, 1926: 13 (D. aleppense var.) [Syria: Aleppo]

latebivittatum Pic, 1931a: 1 (D. aleppense var.) [?]

internenotatum Pic, 1931c: 9 (D. aleppense var.) [Syria: Aleppo]

brunneosericans Breuning, 1946: 101 (D. halepense m.) [Syria: Aleppo]

akbesianum Breuning, 1946: 101 (D. halepense m.) [Turkey: Hatay: Akbez]

pseudosimile Breuning, 1946: 102 (D. halepense m.) [Turkey: Hatay: Akbez]

pseudomardinense Breuning, 1946: 102 (D. halepense m.) [Turkey: Hatay: Akbez]

dorsoinvittatum Breuning, 1948: 58 (D. halepense m.) [Turkey: Hatay: Akbez]

Provincial distribution in Turkey: TR-A: HT (Breuning 1946, 1948; Önalp 1991; Löbl and Smetana 2010; Özdikmen 2010; Danilevsky 2015a)

Range: A: SY TR 
*D. halepense sehitkamilense Özdikmen, Mercan and Cihan, 2012a: 604

Type information: Holotype $\hat{\jmath}$, collection H. Özdikmen, Zoological Museum of Gazi University, Ankara [type locality 'Şehitkamil' (Turkey: Gaziantep)]

Provincial distribution in Turkey: TR-A: GA KL (Breuning 1962a; Braun 1978a; Özdikmen 2010, 2012; Özdikmen et al. 2012a; Danilevsky 2015a)

Range: A: TR

*D. hampii Mulsant and Rey, 1863: 157

*D. hampii aureovittatum Kraatz, 1873: 81 (D. aureovittatum)

Type information: Syntypes $\hat{\partial} \hat{\partial}$, ex collection G. Kraatz, Deutsches Entomologisches Institut, Eberswalde [type locality 'Kleinasien' (Turkey)]

Provincial distribution in Turkey: TR-A: SV (Kraatz 1873; Breuning 1962a; Löbl and Smetana 2010; Özdikmen 2010, 2012; Danilevsky 2015a)

Range: A: TR

*D. hampii hampii Mulsant and Rey, 1863: 157

Type information: Holotype, ex collection Petri Pellet, Muséum d'Histoire Naturelle de Perpignan [type locality 'La Perse' undoubtedly mislabeled, should be Turkey]

claresuturale Breuning, 1946: 109 (D. hampei m.) [Turkey: Amasya]

discofasciatum Breuning, 1946: 110 (D. hampei m.) [Turkey: Amasya]

Provincial distribution in Turkey: TR-A: AM EZ KAR TO (Breuning 1946, 1962a;

Plavilstshikov 1958; Löbl and Smetana 2010; Özdikmen 2010; Danilevsky 2015a)

Range: A: TR

*D. hanneloreae Bernhauer and Peks, 2014a: 107

Type information: Holotype $\hat{\sigma}$, collection Dieter Bernhauer, Bad Schwalbach [type locality 'S Bulanık' (Turkey: Muş)]

Provincial distribution in Turkey: TR-A: MU (Bernhauer and Peks 2014a; Danilevsky 2015a) Range: A: TR

$$
\text { *D. heinzi Breuning, 1964a: } 1 \text { (D. cinerarium ssp.) }
$$

Type information: Holotype $\hat{\sigma}$, ex collection S. Breuning, Muséum d'Histoire Naturelle de Genève [type locality 'Eğribel pass' (Turkey: Giresun)]

Provincial distribution in Turkey: TR-A: Gl (Breuning 1964a; Braun 1978a, 1979; Löbl and Smetana 2010; Özdikmen 2010, 2012; Danilevsky 2015a)

Range: A: TR

\section{D. hellmanni Ganglbauer, 1884: 486}

Type information: Syntypes $\widehat{\partial} \widehat{\partial}$ and $q+q$, ex collection L. Ganglbauer, Naturhistorisches Museum Wien [type locality 'Persien' (Iran)]

ingens Breuning, 1946: 100 (D. hellmanni m.) [Turkey: Van]

postjunctum Breuning, 1946: 100 (D. hellmanni m.) [Turkey: Van]

Provincial distribution in Turkey: TR-A: HA MR MU TU VA (Breuning 1946; Plavilstshikov 1958; Fuchs and Breuning 1971; Holzschuh 1980; Löbl and Smetana 2010; Özdikmen 2010; Sama et al. 2012; Danilevsky 2015a)

Range: A: IN IQ TR 
*D. holtzi Pic, 1905a: 115 (D. culminicola ssp.)

Type information: Holotype, ex collection M. Pic, Muséum National d'Histoire Naturelle, Paris [type locality 'Taurus' (Turkey: ?içel)]

Provincial distribution in Turkey: TR-A: IC (Pic 1905a; Bodemeyer 1906; Breuning 1962a; Löbl and Smetana 2010; Özdikmen 2010, 2012; Danilevsky 2015a)

Range: A: TR

*D. iconiense K. Daniel, 1900: 140

Type information: Syntypes $\hat{\partial} \hat{\partial}$, ex collection K. Daniel, Deutsches Entomologisches Institut, Eberswalde [type locality 'Asia Minor' (Turkey)]

semisetosum Jakovlev, 1901: 85 [Turkey: Konya]

fulvovestitum Pic, 1903a: 5 (D. iconiense var.) [Turkey]

albicolle Breuning, 1943: 89 [Turkey: Konya]

posticeapertum Breuning, 1946: 109 (D. iconiense m.) [Turkey: Konya]

Provincial distribution in Turkey: TR-A: AK CO KIR KN KY YO (Daniel 1900; Jakovlev 1901;

Pic 1903a; Breuning 1943, 1946, 1962b; Braun 1978a, 1979; Löbl and Smetana 2010; Özdikmen 2010, 2012; Lazarev 2011; Danilevsky 2015a; Özdikmen and Kaya 2015a)

Range: A: TR

*D. infernale Mulsant and Rey, 1863: 158

*D. infernale asperatum Breuning, 1947: 169

Type information: Holotype $q$, ex collection S. Breuning, Muséum d'Histoire Naturelle de Genève [type locality 'Diyarbakır' (Turkey)]

Provincial distribution in Turkey: TR-A: DI (Breuning 1947, 1962a; Löbl and Smetana 2010; Özdikmen 2010, 2012; Danilevsky 2015a)

Range: A: TR

*D. infernale edremitense Breuning, 1966a: 20

Type information: Holotype $\hat{\jmath}$, Institut Royal des Sciences Naturelles de Belgique [type locality 'Edremit' (Turkey: Balıkesir)]

Provincial distribution in Turkey: TR-A: BL (Breuning 1966a; Löbl and Smetana 2010; Özdikmen 2010, 2012; Danilevsky 2015a)

Range: A: TR

*D. infernale infernale Mulsant and Rey, 1863: 158

Type information: Holotype, ex collection Petri Pellet, Muséum d'Histoire Naturelle de Perpignan [type locality 'La Perse' undoubtedly mislabeled, should be Turkey]

rugosum J. Thomson, 1867: 46 [?Russia: Siberia]

revestitum K. Daniel, 1900: 140 (D. infernale var.) [Turkey]

immutatum Pic, 1903b: 171 (D. infernale var.) [Turkey: İzmir: Bozdağ]

costidorsum Heyrovský, 1932: 103 (D. infernale ab.) [Turkey: Eskişehir]

subrevestitum Breuning, 1946: 115 (D. infernale m.) [Turkey: Bilecik]

murinum Breuning, 1946: 115 (D. infernale m.) [Turkey: İzmir: Bozdağ]

irrevestitum Breuning, 1946: 115 (D. infernale m.) [Turkey: Bilecik]

Provincial distribution in Turkey: TR-A: AM AN ANT BI BU CO ES IC IZ KN KU NI SV US; TR-

E: KK (Ganglbauer 1884; Bodemeyer 1900; 1906; Daniel 1900; Pic 1903b; Daniel and

Daniel 1903; Heyrovský 1932; Breuning 1946; 1962a; Demelt 1963; Braun 1978a; Önalp 
1990; Adlbauer 1992; Özdikmen and Hasbenli 2004a; Özdikmen 2006; 2010, 2012; Özdikmen et al. 2009; Löbl and Smetana 2010; Sama et al. 2012; Şenyüz and Özdikmen 2013; Danilevsky 2015a).

Range: E: TR A: TR

*D. infernale miminfernale Breuning, 1970: 98 (D. miminfernale)

Type information: Holotype $\widehat{\partial}$, Muséum National d'Histoire Naturelle, Paris [type locality 'Binboğa Mt.: Göksun' (Turkey: Kahramanmaraş)]

Provincial distribution in Turkey: TR-A: KA KY (Breuning 1970; Braun 1978a; Özdikmen 2010, 2012; Danilevsky 2015a)

Range: A: TR

*D. inikliense Bernhauer and Peks, 2010b: 208

Type information: Holotype $\widehat{\partial}$, collection Dieter Bernhauer, Bad Schwalbach [type locality 'İznik' (Turkey: Bursa)]

Provincial distribution in Turkey: TR-A: BS (Bernhauer and Peks 2010; Özdikmen 2012; Danilevsky 2015a)

Range: A: TR

*D. inopinatum Pesarini and Sabbadini, 2013: 51

Type information: Holotype $\hat{\jmath}$, collection Carlo Pesarini and Andrea Sabbadini, Milano [type locality 'Bozdağ' (Turkey: İzmir)]

Provincial distribution in Turkey: TR-A: IZ (Pesarini and Sabbadini 2013; Danilevsky 2015a)

Range: A: TR

*D. inspersum Holzschuh, 1982: 76

Type information: Holotype $\hat{\sigma}$, collection Carolus Holzschuh, Villach [type locality 'Kuzgunkıran pass' (Turkey: Van)]

Provincial distribution in Turkey: TR-A: VA (Holzschuh 1982; Löbl and Smetana 2010; Özdikmen 2010, 2012; Danilevsky 2015a)

Range: A: TR

*D. intrusum Pesarini and Sabbadini, 2009: 26

Type information: Holotype $\hat{\sigma}$, collection Carlo Pesarini and Andrea Sabbadini, Milano [type locality 'SW Gelibolu' (Turkey: Edirne)]

Provincial distribution in Turkey: TR-E: ED (Pesarini and Sabbadini 2009; Özdikmen 2012; Danilevsky 2015a)

Range: E: TR

*D. ispartense Breuning, 1960: 1

Type information: Holotype $q$, ex collection H. Coiffait, Muséum National d'Histoire Naturelle, Paris [type locality 'Bozanönü' (Turkey: Isparta)]

Provincial distribution in Turkey: TR-A: IP (Breuning 1960, 1962a; Özdikmen 2010, 2012; Danilevsky 2015a)

Range: A: TR 
*D. ispirense Bernhauer and Peks, 2013b: 328

Type information: Holotype $\hat{\sigma}$, collection Dieter Bernhauer, Bad Schwalbach [type locality 'Ispir' (Turkey: Erzurum)]

Provincial distribution in Turkey: TR-A: EZ (Bernhauer and Peks 2013b; Danilevsky 2015a) Range: A: TR

*D. ivani Pesarini and Sabbadini, 2011: 47

Type information: Holotype $\widehat{\sigma}$, collection Carlo Pesarini and Andrea Sabbadini, Milano) [type locality 'Kardeşler' (Turkey: Muş)] A: TR

Provincial distribution in Turkey: TR-A: BN MU (Pesarini and Sabbadini 2011; Özdikmen 2012; Rapuzzi and Sama 2012; Danilevsky 2015a)

Range: A: TR

*D. jacovleviellum Plavilstshikov, 1951: 120

Type information: Lectotype $\widehat{\partial}$, ex collection N. N. Plavilstshikov, Zoological Museum, University of Moscow [type locality 'Erzurum env.' (Turkey)]

Provincial distribution in Turkey: TR-A: EZ KAR (Plavilstshikov 1951; Breuning 1962a; Braun 1978a; Danilevsky 2009, 2015a; Löbl and Smetana 2010; Özdikmen 2010, 2012) Range: A: TR

*D. janatai Kadlec, 2006: 11

Type information: Holotype $\widehat{\jmath}$, collection S. Kadlec, Litvinov [type locality 'Karaoğlan Mt.' (Turkey: Tunceli)]

Provincial distribution in Turkey: TR-A: TU (Kadlec 2006; Löbl and Smetana 2010; Özdikmen 2010, 2012; Danilevsky 2015a)

Range: A: TR

\section{D. javeti Kraatz, 1873: 91}

Type information: Syntypes $\widehat{\jmath} \hat{\delta}$ and $q$, , ex collection G. Kraatz, Deutsches Entomologisches Institut, Eberswalde [type locality 'Syria: Akbez', should be 'Akbez' (Turkey: Hatay)]

destinoi Fairmaire, 1884: 173 [Turkey: Hatay: Akbez]

univestitum Pic, 1903a: 5 (D. destinoi var.) [Turkey: Hatay: Akbez]

tabense Pic, 1931a: 1 (D. aleppense var.) [Turkey: Gaziantep ('Ain-Tab'), not Syria]

Provincial distribution in Turkey: TR-A: AD GA HT IC OS (Fairmaire 1884; Ganglbauer 1884; Pic 1903a, 1931a; Breuning 1946, 1962a; Fuchs and Breuning 1971; Önalp 1991; Löbl and Smetana 2010; Özdikmen 2010, 2012; Danilevsky 2015a; Özdikmen and Kaya 2015b) Range: A: SY TR

D. johannisfranci Pesarini and Sabbadini, 2007: 40

Type information: Holotype $\hat{\jmath}$, Museo Civico di Storia Naturale, Milano [type locality 'Tracia: Monastraki' (Greece)]

Provincial distribution in Turkey: TR-E: ED (Pesarini and Sabbadini 2007; Löbl and Smetana 2010; Özdikmen 2010; Danilevsky 2015a)

Range: E: GR TR 
*D. karacaorenense Bernhauer and Peks, 2012: 211

Type information: Holotype $\hat{\partial}$, collection Dieter Bernhauer, Bad Schwalbach [type locality 'İmranlı: Karacaören' (Turkey: Sivas)]

karacaoerenense Bernhauer and Peks, 2013b: 336 [unjustified emendation]

Provincial distribution in Turkey: TR-A: ER SV (Bernhauer and Peks 2012, 2013b; Danilevsky 2015a)

Range: A: TR

*D. karamanense Özdikmen and Koçak, 2013: 672

Type information: Holotype $\widehat{\partial}$, collection Özgur Koçak, Karaman [type locality 'KılbasanKaradağ: Halisgömü plateau' (Turkey: Karaman)]

Provincial distribution in Turkey: TR-A: KM (Özdikmen and Koçak 2013; Danilevsky 2015a) Range: A: TR

*D. karsense Suvorov, 1915: 118

Type information: Holotype $\hat{\jmath}$, ex collection G. Suvorov, Zoological Museum, University of Moscow [type locality 'Kağızman' (Turkey: Kars)]

Provincial distribution in Turkey: TR-A: AR KAR (Suvorov 1915; Plavilstshikov 1958; Breuning 1962a; Löbl and Smetana 2010; Özdikmen 2010, 2012; Danilevsky 2015a) Range: A: TR

*D. kartaldagense Bernhauer and Peks, 2013a: 314

Type information: Holotype $\hat{\partial}$, collection Dieter Bernhauer, Bad Schwalbach [type locality 'Kartaldağ' (Turkey: Çanakkale)]

Provincial distribution in Turkey: TR-A: CA (Bernhauer and Peks 2013a; Danilevsky 2015a) Range: A: TR

D. kasikoporanum Pic, 1902b: 10

Type information: Holotype $\hat{\sigma}$, ex collection M. Pic, Muséum National d'Histoire Naturelle, Paris [type locality 'Kazkoparan' (Turkey: Iğdır)]

Provincial distribution in Turkey: TR-A: AR IG (Pic 1902b; Plavilstshikov 1958; Breuning 1962a; Lazarev 2009; Löbl and Smetana 2010; Özdikmen 2010, 2012; Danilevsky 2015a) Range: A: AR TR

*D. kazanciense Bernhauer and Peks, 2010: 197

Type information: Holotype $\widehat{\partial}$, collection Dieter Bernhauer, Bad Schwalbach [type locality 'N Anamur: N Akpınar, Kazancı' (Turkey: İçel)]

Provincial distribution in Turkey: TR-A: IC (Bernhauer and Peks 2010; Özdikmen 2012; Danilevsky 2015a)

Range: A: TR

*D. kindermanni Waltl, 1838: 470

Type information: Holotype $\delta$, ex collection Imre Frivaldszky, Magyar Természettudományi Mûzeum, Budapest [type locality 'Turkei' (Turkey)] griseum Waltl, 1838: 470 [Turkey] fuscoquadriplagiatum Breuning, 1946: 110 (D. kindermanni m.) [Turkey: İzmir] fuscoreductum Breuning, 1946: 110 (D. kindermanni m.) [Turkey: Çanakkale] 
Provincial distribution in Turkey: TR-A: AN CA IZ US (Küster 1846a; Ganglbauer 1884; Breuning 1946, 1962a; Demelt 1963; Önalp 1991; Löbl and Smetana 2010; Özdikmen 2010, 2012; Danilevsky 2015a)

Range: A: TR

*D. kizildagense Bernhauer and Peks, 2012: 212

Type information: Holotype $\hat{\sigma}$, collection Dieter Bernhauer, Bad Schwalbach [type locality 'İmranlı: Kızıldağ' (Turkey: Sivas)]

Provincial distribution in Turkey: TR-A: ER SV (Bernhauer and Peks 2012, 2013b;

Danilevsky 2015a)

Range: A: TR

*D. konradi Bernhauer and Peks, 2012: 210

Type information: Holotype $\widehat{\partial}$, collection Dieter Bernhauer, Bad Schwalbach [type locality 'Kuruca pass' (Turkey: Bingöl)]

Provincial distribution in Turkey: TR-A: BN (Bernhauer and Peks 2012, 2013b; Danilevsky 2015a) Range: A: TR

*D. kraetschmeri Bernhauer, 1988: 98

Type information: Holotype $\hat{\sigma}$, collection Dieter Bernhauer, Bad Schwalbach [type locality 'Akseki' (Turkey: Antalya)]

Provincial distribution in Turkey: TR-A: ANT (Bernhauer 1988; Löbl and Smetana 2010; Özdikmen 2010, 2012; Danilevsky 2015a)

Range: A: TR

*D. kurdistanum Breuning, 1944: 12

Type information: Holotype $\widehat{\partial}$, ex collection S. Breuning, Muséum d'Histoire Naturelle de Genève [type locality 'Diyarbakır' (Turkey)]

rufulipes Breuning, 1963: 36 (D. kurdistanum m.) [Turkey: Batman: Sason Mts.]

Provincial distribution in Turkey: TR-A: ADY BA BN DI (Breuning 1944, 1962a, 1963; Fuchs and Breuning 1971; Löbl and Smetana 2010; Özdikmen 2010, 2012; Sama et al. 2012; Danilevsky 2015a)

Range: A: TR

*D. kurucanum Holzschuh, 2007: 249

Type information: Holotype $\hat{\partial}$, collection Carolus Holzschuh, Villach [type locality 'Kuruca pass' (Turkey: Elazığ)]

Provincial distribution in Turkey: TR-A: EL (Holzschuh 2007; Löbl and Smetana 2010; Özdikmen 2010, 2012; Danilevsky 2015a)

Range: A: TR

*D. Iadikanum Braun, 1976a: 173 [RN]

Type information: Holotype $\widehat{\partial}$, Muséum National d'Histoire Naturelle, Paris [type locality 'Ladik' (Turkey: Samsun)]

ladikense Breuning, 1975: 10 [HN] [Turkey: Samsun: Ladik]

paraladikense Breuning, 1977: 60 [RN] [Turkey: Samsun: Ladik]

Provincial distribution in Turkey: TR-A: SM (Breuning 1975, 1977; Braun 1976a; Löbl and Smetana 2010; Özdikmen 2010, 2012; Bernhauer and Peks 2013b; Danilevsky 2015a) Range: A: TR 


\section{D. laeve Faldermann, 1837: 278 \\ *D. Iaeve micula Plavilstshikov, 1937: 26 (D. micula)}

Type information: Lectotype, ex collection Dobrovl. > N. N. Plavilstshikov, Zoological Museum, University of Moscow [type locality 'Oltu' (Turkey: Erzurum)]

Provincial distribution in Turkey: TR-A: EZ (Plavilstshikov 1937; Breuning 1962a; Danilevsky and Murzin 2009; Löbl and Smetana 2010; Özdikmen 2010, 2012; Danilevsky 2015a)

Range: A: TR

Other known subspecies: D. laeve laeve Faldermann, 1837: $278 \mathrm{~A}$ : IN; D. laeve hyrcanum Jakovlev, 1899b: 64 A: IN; D. laeve vladimiri Danilevsky and Murzin, 2009: 13 A: AR

*D. Iameeri Théry, 1896: 109

Type information: Syntypes $2 \widehat{\widehat{\partial}}$ and $2 \circ+$, ex collection A. Théry > M. Pic, Muséum National d'Histoire Naturelle, Paris [type locality 'Kastamonu' (Turkey)]

Provincial distribution in Turkey: TR-A: KS (Théry 1896; Breuning 1946, 1962a; Demelt 1967; Önalp 1991; Löbl and Smetana 2010; Özdikmen 2010, 2012; Danilevsky 2015a) Range: A: TR

*D. ledouxi Breuning, 1974b: 65

Type information: Holotype ${ }_{+}$, Muséum National d'Histoire Naturelle, Paris [type locality 'Ilıca' (Turkey: Erzurum)]

Provincial distribution in Turkey: TR-A: EZ (Breuning 1974b; Löbl and Smetana 2010; Özdikmen 2010, 2012; Danilevsky 2015a)

Range: A: TR

*D. linderi Tournier, 1872: 285

Type information: Holotype $q$, ex collection H. Tournier > M. Pic, Muséum National d'Histoire Naturelle, Paris [type locality 'Spain' but undoubtedly mislabeled, should be Turkey]

kollari Kraatz, 1873: 87 [Turkey: Amasya]

henrici Pic, 1905b: 156 [Turkey: Tokat]

anticepunctatum Breuning, 1946: 109 (D. kollari m.) [Turkey: Tokat]

quadripunctum Breuning, 1946: 109 (D. kollari m.) [Turkey: Tokat]

unipunctum Breuning, 1946: 109 (D. kollari m.) [Turkey: Tokat]

sublinderi Breuning, 1966a: 20 (D. kollari m.) [Turkey: Samsun]

dorsoimmaculipenne Breuning, 1966b: 147 (D. kollari m.) [Turkey: Çorum: Boğazkale:

Yazılıkaya]

Provincial distribution in Turkey: TR-A: AM CO SM TO (Kraatz 1873; Ganglbauer 1884; Pic 1905b; Breuning 1946, 1962a, 1966a, 1966b; Perissinotto and Luchini 1966; Sama 1982; Löbl and Smetana 2010; Özdikmen 2010, 2012; Danilevsky 2015a)

Range: A: TR

\section{D. lineatocolle Kraatz, 1873: 57 (D. femoratum var.)}

Type information: Syntypes $\widehat{\partial} \widehat{\partial}$ and $q \mathcal{+}$, ex collection G. Kraatz, Deutsches Entomologisches Institut, Eberswalde [type locality 'Salonica' (Greece)] atrofemoratum Pic, 1917b: 7 (D. lineatocolle var.) [Macedonia] sinuaticolle Pic, 1927: 6 [Greece: Salonica] 
riveti Pic, 1927: 162 (D. lineatocolle var.) [Macedonia]

Provincial distribution in Turkey: TR-E (Önalp 1991; Özdikmen 2010)

Range: E: AL BU GR MC TR YU

*D. lodosi Sabbadini and Pesarini, 1992: 29

*D. lodosi abanozense Bernhauer and Peks, 2010: 199

Type information: Holotype $\hat{\sigma}$, collection Dieter Bernhauer, Bad Schwalbach [type locality 'Anamur: Abanoz' (Turkey: İçel)]

Provincial distribution in Turkey: TR-A: IC (Bernhauer and Peks 2010; Özdikmen 2012; Danilevsky 2015a)

Range: A: TR

*D. Iodosi lodosi Sabbadini and Pesarini, 1992: 29

Type information: Holotype $\hat{\jmath}$, collection Carlo Pesarini and Andrea Sabbadini, Milano [type locality 'Bucakkışla' (Turkey: Karaman)]

Provincial distribution in Turkey: TR-A: KM (Sabbadini and Pesarini 1992; Bernhauer and Peks 2010; Löbl and Smetana 2010; Özdikmen 2010, 2012; Danilevsky 2015a)

Range: A: TR

*D. Iohsei Braun, 1976b: 254

Type information: Holotype $\hat{\delta}$, collection Walter Braun, Tübingen [type locality 'Karaman' (Turkey)]

Provincial distribution in Turkey: TR-A: IC KN KM (Braun 1976b, 1978a; Sama 1982; Adlbauer 1992; Löbl and Smetana 2010; Özdikmen 2010, 2012; Turgut and Özdikmen 2010; Pesarini and Sabbadini 2013; Danilevsky 2015a)

Range: A: TR

*D. longulum Breuning, 1943: 89

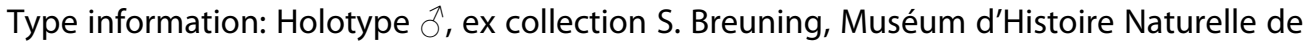
Genève [type locality 'Bozdağ' (Turkey: İzmir)]

Provincial distribution in Turkey: TR-A: IZ (Breuning 1943, 1962a; Löbl and Smetana 2010; Özdikmen 2010, 2012; Danilevsky 2015a)

Range: A: TR

\section{Iugubre Kraatz, 1873: 41}

\section{D. lugubre lugubre Kraatz, 1873: 41}

Type information: Lectotype $\hat{\sigma}$, ex collection G. Kraatz, Deutsches Entomologisches Institut, Eberswalde [type locality 'Salonica' (Greece)]

salonicum Pic, 1916: 22 [Greece: Salonica]

discosulcatum Pic, 1926: 1 (D. salonicum var.) [Macedonia]

sparsepilosum Pic, 1926: 13 (D. lugubre var.) [Greece: Salonica]

pseudolugubre Breuning, 1943: 96 [Macedonia: 'Karatschköi' = Dioni]

Provincial distribution in Turkey: TR-A: AM SV; TR-E (Önalp 1990; Özdikmen and Hasbenli 2004a; Özdikmen 2010)

Range: E: ?BU GR MC TR A: TR 
Other known subspecies: D. lugubre meteorum Breuning, 1969: $42 \mathrm{E}: \mathrm{GR}$; D. lugubre minkovae Heyrovský, 1962: $225 \mathrm{E}: \mathrm{BU}$; D. lugubre parinfernale Breuning, 1975: $10 \mathrm{E}: \mathrm{GR} ; \mathrm{D}$. lugubre pelionense Breit, 1923: 145 E: GR; D. lugubre thessalicum Pic, 1916: 22 E: GR

*D. maceki Holzschuh, 1995: 41

Type information: Holotype $\widehat{\partial}$, collection J. Macek [type locality 'Abant' (Turkey: Bolu)] Provincial distribution in Turkey: TR-A: BO (Breuning 1943, 1962a; Holzschuh 1995; Löbl and Smetana 2010; Özdikmen 2010, 2012; Danilevsky 2015a)

Range: A: TR

\section{D. margheritae Breuning, 1964b: 32}

Type information: Holotype $\hat{\sigma}$, collection Dr. Franco Tassi, Rome [type locality 'Epire (loánnina): Pinde Mts.' (Greece)]

katarense Breuning, 1969: 42 (D. margheritae m.) [Greece: Katara]

Provincial distribution in Turkey: TR-A: CA; TR-E (Adlbauer 1988; Althoff and Danilevsky 1997; Özdikmen 2010; Danilevsky 2015a)

Range: E: GR TR A: TR

*D. martini Bernhauer, 1988: 100

Type information: Holotype $\widehat{\sigma}$, collection Dieter Bernhauer, Bad Schwalbach [type locality 'Nallıhan' (Turkey: Ankara)]

Provincial distribution in Turkey: TR-A: AN (Bernhauer 1988; Löbl and Smetana 2010; Özdikmen 2010, 2012; Danilevsky 2015a)

Range: A: TR

*D. menradi Holzschuh, 1989: 172

Type information: Holotype $\widehat{\partial}$, collection Carolus Holzschuh, Villach [type locality 'Afşin' (Turkey: Kahramanmaraş)]

Provincial distribution in Turkey: TR-A: KA MA (Holzschuh 1989; Löbl and Smetana 2010; Özdikmen 2010, 2012; Pesarini and Sabbadini 2013; Danilevsky 2015a)

Range: A: TR

*D. merkli Ganglbauer, 1884: 506

Type information: Syntypes $\hat{\sigma}$ and $q$, ex collection L. Ganglbauer, Naturhistorisches Museum Wien [type locality 'nordwestlichen Kleinasien: Akdağ (?Simav)' (Turkey: ? Kütahya)]

humerointerruptum Breuning, 1946: 109 (D. merkli m.) [Turkey: ?Kütahya: ?Simav: Akdağ]

Provincial distribution in Turkey: TR-A: KU (Ganglbauer 1884; Breuning 1946, 1962a; Löbl and Smetana 2010; Özdikmen 2010, 2012; Danilevsky 2015a)

Range: A: TR

D. mesopotamicum Breuning, 1944: 12

Type information: Holotype $\widehat{\partial}$, ex collection S. Breuning, Muséum d'Histoire Naturelle de Genève [type locality 'Mesopotamia: Ras Al-Ain' (Iraq)]

submardinense Breuning, 1946: 109 (D. mesopotamicum m.) [Iraq: Mesopotamia: Ras Al-Ain] 
Provincial distribution in Turkey: TR-A: MR SU (Breuning 1962a; Braun 1978a; Löbl and Smetana 2010; Özdikmen 2010; Danilevsky 2015a)

Range: A: IQ TR

*D. micans J. Thomson, 1867: 61

*D. micans micans J. Thomson, 1867: 61

Type information: Holotype $\hat{\partial}$, ex collection J. Thomson > R. Oberthür, Muséum National d'Histoire Naturelle, Paris [type locality 'Armenien' undoubtedly mislabeled, should be Turkey]

macropus Kraatz, 1873: 99 [Turkey: Amasya]

sericatulum Kraatz, 1873: 98 [?Caucasus, Turkey: Ankara, Bursa]

obscurans Pic, 1892a: 91 (D. macropus var.) [Turkey: Amasya]

amasinum Pic, 1898: 58 [Turkey: Amasya]

atripes Reitter, 1900: 88 (D. sericatum var.) [Turkey: Amasya]

tokatense Pic, 1901: 12 (D. impressicolle var.) [Turkey: Tokat]

corallicorne Pic, 1904: 4 (D. sericatum var.) [Turkey: Ankara]

subreductum Pic, 1942a: 2 (D. sericatum var.) [Turkey]

subobesum Pic, 1942a: 2 [Turkey: Amasya]

Provincial distribution in Turkey: TR-A: AM AN BS CA CO IZ TO YO (Kraatz 1873; Pic 1892a, 1898, 1901, 1904, 1942a; Reitter 1900; Breuning 1962a; Demelt 1963; Perissinotto and Luchini 1966; Breuning and Villiers 1967; Fuchs and Breuning 1971; Gül-Zümreoğlu 1975; Holzschuh 1980; Löbl and Smetana 2010; Özdikmen 2010; Danilevsky 2015a; Özdikmen and Kaya 2015d)

Range: A: TR

*D. micans susheriense Breuning, 1970: 97 (D. sinopense ssp.)

Type information: Holotype $\widehat{\partial}$, Muséum National d'Histoire Naturelle, Paris [type locality 'Suşehri' (Turkey: Sivas)]

Provincial distribution in Turkey: TR-A: SV (Breuning 1970; Löbl and Smetana 2010; Özdikmen 2010, 2012; Danilevsky 2015a; Özdikmen and Kaya 2015d)

Range: A: TR

D. mniszechi Kraatz, 1873: 39

D. mniszechi mniszechi Kraatz, 1873: 39

Type information: Syntypes $\hat{\sigma}$ and $q$, ex Musaeo Mniszech > R. Oberthür, Muséum National d'Histoire Naturelle, Paris [type locality 'Caucasus']

rugulosum Breuning, 1946: 96 (D. mniszechi m.) [Turkey: Iğdır: Kazkoparan]

Provincial distribution in Turkey: TR-A: AG EZ IG KAR SV (Breuning 1946; Plavilstshikov 1958; Özdikmen and Hasbenli 2004a; Löbl and Smetana 2010; Özdikmen 2010; Lazarev 2014; Danilevsky 2015a)

Range: A: AR TR

D. mniszechi cavernosum Lazarev, 2014: 701

Type information: Holotype $\widehat{\jmath}$, ex collection M. L. Danilevsky, Collection of Zoological Institute of the Russian Academy of Sciences, Saint-Petersbourg [type locality Arteni (Armenia)] Provincial distribution in Turkey: TR-A: ART IG KAR (Breuning 1946; Lazarev 2014; Danilevsky 2015a) 
Range: A: AR TR

Other known subspecies: D. mniszechi georgianum Lazarev, 2014: 701 A: GG

*D. muchei Breuning, 1962b: 38

Type information: Holotype $\widehat{\jmath}$, collection H. Muche [type locality 'Çankırı' (Turkey)] plurivittipenne Breuning, 1962b: 39 (D. muchei m.) [Turkey: Çankırı] parescherichi Breuning, 1966b: 146 [Turkey: Çorum: Boğazkale]

Provincial distribution in Turkey: TR-A: CN CO KS KY YO (Breuning 1962a, 1962b, 1966b; Perissinotto and Luchini 1966; Demelt 1967; Braun 1978a, 1979; Holzschuh 1980; Özdikmen 2010, 2012; Özdikmen and Kaya 2015a)

Range: A: TR

*D. multimaculatum Pic, 1932: 22

Type information: Holotype, ex collection Leo Heyrovský, Musée National de Prague [type locality 'Ödemiş' (Turkey: İzmir)] postbiplagiatum Breuning, 1946: 107 (D. multimaculatum m.) [Turkey: İzmir: Ödemiş] Provincial distribution in Turkey: TR-A: IZ (Pic 1932; Breuning 1946, 1962a; Löbl and Smetana 2010; Özdikmen 2010, 2012; Danilevsky 2015a)

Range: A: TR

*D. murzini Danilevsky, 2015a: 221

Type information: Holotype $\hat{\partial}$, ex collection M. L. Danilevsky, Collection of Zoological Museum, University of Moscow [type locality 'Isspir: Yukarıözbağ' (Turkey: Erzurum)]

Provincial distribution in Turkey: TR-A: EZ (Danilevsky, 2015b)

Range: A: TR

*D. musense Bernhauer and Peks, 2014a: 109

Type information: Holotype $\widehat{\partial}$, collection Dieter Bernhauer, Bad Schwalbach [type locality 'S Bulanık' (Turkey: Muş)]

Provincial distribution in Turkey: TR-A: MU (Bernhauer and Peks 2014a; Danilevsky 2015a) Range: A: TR

*D. narlianum Özdikmen, Mercan and Cihan, 2012b: 550

Type information: Holotype $\hat{\partial}$, collection H. Özdikmen, Zoological Museum of Gazi University, Ankara [type locality 'Narlı' (Turkey: Kahramanmaraş)]

Provincial distribution in Turkey: TR-A: KA (Özdikmen 2012; Özdikmen et al. 2012b, 2012c; Danilevsky 2015a)

Range: A: TR

*D. nigrostriatum Adlbauer, 1982: 104

Type information: Holotype $\hat{\partial}$, collection Karl Adlbauer, Graz [type locality 'N Tunceli' (Turkey)]

Provincial distribution in Turkey: TR-A: TU (Adlbauer 1982, 1988; Löbl and Smetana 2010; Özdikmen 2010, 2012; Sama et al. 2012; Danilevsky 2015a)

Range: A: TR

*D. nihalae Rapuzzi and Sama, 2012: 671

Type information: Holotype $\widehat{\jmath}$, collection Gianfranco Sama, Cesena [type locality 'Zara' (Turkey: Sivas)] 
Provincial distribution in Turkey: TR-A: SV (Özdikmen 2012; Rapuzzi and Sama 2012; Danilevsky 2015a)

Range: A: TR

*D. niksarense Bernhauer and Peks, 2013b: 327

Type information: Holotype $\hat{\sigma}$, collection Dieter Bernhauer, Bad Schwalbach [type locality 'Niksar' (Turkey: Tokat)]

Provincial distribution in Turkey: TR-A: TO (Bernhauer and Peks 2013b; Danilevsky 2015a) Range: A: TR

D. nitidum Motschulsky, 1838: 185

Type information: Holotype, ex collection V. I. Motschulsky, Zoological Museum, University of Moscow [type locality 'Armenia'] suturatum Ferrari, 1864: 481 [Georgia: Achalzik]

Provincial distribution in Turkey: TR-A: ART EZ KAR YO (Plavilstshikov 1958; Breuning 1962a; Özbek 1978; Adlbauer 1988; Önalp 1991; Özdikmen and Hasbenli 2004a; Löbl and Smetana 2010; Özdikmen 2010; Danilevsky 2015a)

Range: $A$ : $A B$ AR GG TR

*D. nobile Hampe, 1852: 313

Type information: Syntypes, ex collection Moritz Wagner, Museum für Naturkunde der Humboldt-Universität zu Berlin [type locality 'W Persien', undoubtedly mislabeled, should be 'Erzurum env.' (Turkey)]

maculatum Breuning, 1946: 119 (D. nobile m.) [Turkey: Kurdistan]

Provincial distribution in Turkey: TR-A: BT EZ VA (Breuning 1946, 1962a; Plavilstshikov 1958; Villiers 1967; Braun 1978a; Sama 1982; Önalp 1991; Özdikmen and Hasbenli 2004a; Löbl and Smetana 2010; Özdikmen 2010, 2012; Pesarini and Sabbadini 2011; Danilevsky 2015a)

Range: A: TR

D. nodicorne Tournier, 1872: 287

Type information: Syntypes $2 \hat{\partial} \widehat{\partial}$ and $q$, ex collection $H$. Tournier $>$ M. Pic, Muséum National d'Histoire Naturelle, Paris [type locality 'Taurus' (Turkey)] maculereductum Breuning, 1962a: 532 (D. quadrimaculatum m.) [Greece: Lesbos Is.] subnodicorne Breuning, 1962a: 532 (D. quadrimaculatum m.) [Greece: Lesbos Is.]

Provincial distribution in Turkey: TR-A: CA IZ Taurus Mts.; TR-E (Breuning 1962a; Krätschmer 1985; Önalp 1990; Althoff and Danilevsky 1997; Löbl and Smetana 2010; Özdikmen 2010, 2012; Danilevsky 2015a)

Range: E: GR TR A: TR

D. obsoletum Kraatz, 1873: 78 (D. graecum var.)

Type information: Holotype $\hat{\sigma}$, ex collection G. Kraatz, Deutsches Entomologisches Institut, Eberswalde [type locality 'Bursa' (Turkey), but not Bursa, according to Kraatz with İstanbul (Turkey) and Greece]

obsoletoides Breuning, 1946: 111 (D. obsoletum m.) [Greece: Thrace: Ferre]

Provincial distribution in Turkey: TR-A: ?BS IS; TR-E: ED IS KK TE (Kraatz 1873; Bodemeyer 1906; Fuchs and Breuning 1971; Braun 1978a; Althoff and Danilevsky 1997; Özdikmen 2010, 2012; Danilevsky 2015a; Özdikmen and Kaya 2015c)

Range: E: BU GR TR A: TR 
*D. obtusum Breuning, 1944: 13

*D. obtusum marashense Breuning, 1948: 59

Type information: Holotype $\widehat{\partial}$, ex collection S. Breuning, Muséum d'Histoire Naturelle de Genève [type locality 'Kahramanmaraş' (Turkey)]

dorsointerruptum Breuning, 1948: 60 (D. obtusum m.) [Turkey: Kahramanmaraş]

Provincial distribution in Turkey: TR-A: KA (Breuning 1948, 1962a; Löbl and Smetana 2010; Özdikmen 2010, 2012; Danilevsky 2015a)

Range: A: TR

*D. obtusum obtusum Breuning, 1944: 13

Type information: Holotype $\widehat{\partial}$, ex collection S. Breuning, Muséum d'Histoire Naturelle de Genève [type locality 'Anatolia' (Turkey)]

Provincial distribution in Turkey: TR-A: ADY DI HA VA (Breuning 1944, 1962a; Fuchs and Breuning 1971; Braun 1978a; Rejzek and Hoskovec 1999; Löbl and Smetana 2010; Özdikmen 2010, 2012; Danilevsky 2015a)

Range: A: TR

\section{*D. oezdurali Önalp, 1988: 361}

Type information: Holotype $\widehat{\jmath}$, collection Birsen Önalp [type locality 'Ahır Mt.' (Turkey: Kahramanmaraş)]

Provincial distribution in Turkey: TR-A: ADY KA (Önalp 1988, 1991; Rejzek and Hoskovec 1999; Pesarini and Sabbadini 1998, 2013; Özdikmen and Okutaner 2006; Löbl and Smetana 2010; Özdikmen 2010, 2012; Sama et al. 2012; Özdikmen and Koçak 2013; Danilevsky 2015a)

Range: A: TR

*D. olympicum Ganglbauer, 1882: 228

*D. olympicum convexum Breuning, 1943: 90

Type information: Holotype $\widehat{\partial}$, ex collection S. Breuning, Muséum d'Histoire Naturelle de Genève [type locality 'Achu Dagh (Simav: Akdağ)' (Turkey: Kütahya)]

Provincial distribution in Turkey: TR-A: KU (Breuning 1943, 1962a; Löbl and Smetana 2010; Özdikmen 2010, 2012; Danilevsky 2015a; Özdikmen and Kaya 2015c)

Range: A: TR

*D. olympicum flavosuturale Krätschmer, 1987: 340

Type information: Holotype $\widehat{\jmath}$, collection Otto Erich Krätschmer, München [type locality 'Mustafakemalpaşa: Gölyazı: Uluabat Lake' (Turkey: Bursa)]

Provincial distribution in Turkey: TR-A: BS (Krätschmer 1987; Löbl and Smetana 2010; Özdikmen 2010, 2012; Danilevsky 2015a; Özdikmen and Kaya 2015c)

Range: A: TR

*D. olympicum olympicum Ganglbauer, 1882: 228

Type information: Lectotype $\hat{\sigma}$, ex collection G. Kraatz, Deutsches Entomologisches Institut, Eberswalde [type locality 'Bursa' (Turkey)]

subalpinum Kraatz, 1873: 78 [Turkey: Bursa: Osmangazi: Uludağ]

oreophilum Ganglbauer, 1884: 500 (D. olympicum var.) [Turkey: Bursa: Osmangazi: Uludağ] 
brussense Breuning, 1946: 112 (D. olympicum m.) [Turkey: Bursa]

quinquefasciatum Breuning, 1946: 112 (D. olympicum m.) [Turkey: Bilecik]

Provincial distribution in Turkey: TR-A: AN BI BS (Kraatz 1873; Ganglbauer 1882, 1884;

Breuning 1946, 1962a; Demelt 1963; Braun 1978a; Adlbauer 1988; Önalp 1991; Sama 1999; Löbl and Smetana 2010; Özdikmen 2010, 2012; Danilevsky 2015a; Özdikmen and Kaya 2015c)

Range: A: TR

*D. ortrudae Braun, 1978b: 185

Type information: Holotype $\widehat{\partial}$, collection Walter Braun, Tübingen [type locality 'Göksun' (Turkey: Kahramanmaraş)]

Provincial distribution in Turkey: TR-A: KA (Braun 1978b; Adlbauer 1988; Löbl and Smetana 2010; Özdikmen 2010, 2012; Danilevsky 2015a)

Range: A: TR

*D. paracinerarium Breuning, 1974a: 149

Type information: Holotype $\hat{\sigma}$, Collection Carolus Holzschuh, Villach [type locality 'Mut: Sertavul pass' (Turkey: İçel)]

Provincial distribution in Turkey: TR-A: IC KM SU (Breuning 1962a, 1974a; Gül-Zümreoğlu 1972; Braun 1978a, 1979; Özdikmen 2012; Özdikmen and Kaya 2015d)

Range: A: TR

*D. pararufipenne Braun, 1976b: 257

*D. pararufipenne pararufipenne Braun, 1976b: 257

Type information: Holotype $\delta^{\lambda}$, collection Walter Braun, Tübingen [type locality 'Abant' (Turkey: Bolu)]

Provincial distribution in Turkey: TR-A: BO (Braun 1976b, 1978a; Özdikmen 2006, 2010, 2012; Özdikmen and Demir 2006; Löbl and Smetana 2010; Bernhauer and Peks 2011 b; Danilevsky 2015a)

Range: A: TR

*D. pararufipenne rassei Braun, 1976b: 261

Type information: Holotype $\hat{\partial}$, collection Walter Braun, Tübingen [type locality 'Gerede' (Turkey: Bolu)]

Provincial distribution in Turkey: TR-A: AN BO (Braun 1976b, 1978a; Adlbauer 1992; Löbl and Smetana 2010; Özdikmen 2010, 2012; Danilevsky 2015a)

Range: A: TR

*D. parilis Pesarini and Sabbadini, 2013: 52

Type information: Holotype $\hat{\partial}$, collection Carlo Pesarini and Andrea Sabbadini, Milano [type locality 'Oluközü' (Turkey: Yozgat)]

Provincial distribution in Turkey: TR-A: YO (Pesarini and Sabbadini 2013; Danilevsky 2015a)

Range: A: TR

*D. pavesii Pesarini and Sabbadini, 1998: 57

Type information: Holotype $\hat{\delta}$, collection Carlo Pesarini and Andrea Sabbadini, Milano [type locality 'Yüksekova' (Turkey: Hakkari)] 
Provincial distribution in Turkey: TR-A: HA (Pesarini and Sabbadini 1998; Löbl and Smetana 2010; Özdikmen 2010, 2012; Danilevsky 2015a)

Range: A: TR

D. pedestre Poda von Neuhaus, 1761: 34 (Cerambyx)

D. pedestre pedestre Poda von Neuhaus, 1761: 34 (Cerambyx)

Type information: Syntypes, ex collection N. Poda [type locality 'ad Graecium' (Greece)] Provincial distribution in Turkey: TR-E: IS (Küster 1846a; Önalp 1990; Özdikmen 2010) Range: E: AL AU BH BU CR CZ HU MC MD PL RO SK SL TR UK YU

Other known subspecies: D. pedestre kaszabi Breuning, 1956: $404 \mathrm{E}: \mathrm{HU}$

*D. peksi Bernhauer, 2010: 190

Type information: Holotype $\widehat{\partial}$, collection Dieter Bernhauer, Bad Schwalbach [type locality 'Anamur' (Turkey: İçel)]

Provincial distribution in Turkey: TR-A: IC (Bernhauer 2010; Özdikmen 2012; Danilevsky 2015a)

Range: A: TR

*D. pergamenum Pesarini and Sabbadini, 2009: 22

Type information: Holotype $\hat{\delta}$, collection Carlo Pesarini and Andrea Sabbadini, Milano [type locality 'Pergama (Bergama)' (Turkey: İzmir)]

Provincial distribution in Turkey: TR-A: IZ (Pesarini and Sabbadini 2009; Özdikmen 2012; Danilevsky 2015a)

Range: A: TR

*D. petrovitzi Heyrovský, 1964: 97

Type information: Holotype $\hat{\jmath}$, ex collection Carl von Demelt, Staatliches Museum für Naturkunde, Stuttgart [type locality 'Çamlıyayla (Namrun)' (Turkey: İçel)]

Provincial distribution in Turkey: TR-A: IC (Heyrovský 1964; Demelt 1967; Löbl and Smetana 2010; Özdikmen 2010, 2012; Danilevsky 2015a)

Range: A: TR

D. pilosipenne Breuning, 1943: 90

*D. pilosipenne kazdagense Özdikmen in Küçükkaykı et al., 2013: 56

Type information: Holotype $\widehat{\partial}$, Department of Biology at Eskişehir Osmangazi University, Eskişehir [type locality 'Edremit, Yayla' (Turkey: Balıkesir)]

Provincial distribution in Turkey: TR-A: BL (Özdikmen in Küçükkaykı et al. 2013; Danilevsky 2015a)

Range: A: TR

Other known subspecies: D. pilosipenne pilosipenne Breuning, 1943: $90 \mathrm{E}: \mathrm{GR}$

*D. piochardi Kraatz, 1873: 85

Type information: Syntypes $\hat{\sigma}$ and $q \mathcal{q}$, ex collection $G$. Kraatz, Deutsches Entomologisches Institut, Eberswalde [type locality 'Samsun-Amasya: Akdağ' (Turkey)] quadripustulatum Ganglbauer, 1884: 457 [Turkey: Çanakkale] elongatum Pic, 1898: 58 (D. piochardi var.) [Turkey: Taurus Mt.] pelops Jakovlev, 1901: 83 [Turkey: Amasya] 
theophilei Pic, 1901: 32 [HN] (D. piochardi var.) [Turkey]

roberti Pic, 1905c: 11 (D. piochardi var.) ['Syria', should be Turkey]

mauricei Plavilstshikov, 1927: 51 [RN]

varievittatum Pic, 1942a: 1 [S Russia, undoubtedly mislabeled]

apicedisparatum Breuning, 1946: 109 (D. piochardi m.) [Turkey: Amasya]

ladikense Breuning andVilliers, 1967: 60 (Pedestredorcadion piochardi var.) [Turkey:

Samsun: Ladik]

Provincial distribution in Turkey: TR-A: AM CA CO SM SN YO (Kraatz 1873; Ganglbauer 1884; Jakovlev 1901; Breuning 1962a; Breuning and Villiers 1967; Braun 1978a, 1979; Önalp 1991; Adlbauer 1992; Löbl and Smetana 2010; Özdikmen 2010, 2012; Lazarev 2011; Danilevsky 2015a)

Range: A: TR

*D. pittinorum Pesarini and Sabbadini, 1998: 48

Type information: Holotype $\hat{\sigma}$, collection Carlo Pesarini and Andrea Sabbadini, Milano [type locality 'Çorum' (Turkey)]

Provincial distribution in Turkey: TR-A: CO (Pesarini and Sabbadini 1998, 2013; Löbl and Smetana 2010; Özdikmen 2010, 2012; Danilevsky 2015a)

Range: A: TR

*D. pluto J. Thomson, 1867: 47

Type information: Holotype $\delta$, ex collection J. Thomson > R. Oberthür, 1952, Muséum National d'Histoire Naturelle, Paris [type locality 'Siberia' but undoubtedly mislabeled, should be Turkey]

Provincial distribution in Turkey: TR-A: AM ES EZ SA SM (Ganglbauer 1884; Breuning and Villiers 1967; Braun 1978a; Önalp 1990; Löbl and Smetana 2010; Özdikmen 2010, 2012; Danilevsky 2015a)

Range: A: TR

*D. poleti Breuning, 1948: 59

Type information: Holotype $\widehat{\partial}$, ex collection S. Breuning, Muséum d'Histoire Naturelle de Genève [type locality 'Caria: Babadağ' (Turkey: Denizli)]

Provincial distribution in Turkey: TR-A: DE (Breuning 1948, 1962a; Löbl and Smetana 2010; Özdikmen 2010, 2012; Danilevsky 2015a)

Range: A: TR

*D. postalbosuturale Özdikmen and Koçak, 2015: 439

Type information: Holotype $\hat{\sigma}$, collection Ö. Koçak, Karaman [type locality 'Elmaşehir district' (Turkey: Karaman)]

Provincial distribution in Turkey: TR-A: KM (Özdikmen and Koçak 2015)

Range: A: TR

*D. praetermissum Pesarini and Sabbadini, 1998: 47

*D. praetermissum mikhaili Özdikmen, 2010: 457

Type information: Holotype $\delta$, Entomology Department of Eastern Mediterranean Forestry Research Institute, İçel [type locality 'Abant' (Turkey: Bolu)]

Provincial distribution in Turkey: TR-A: BO (Özdikmen 2010, 2011, 2012; Danilevsky 2015a)

Range: A: TR 
*D. praetermissum praetermissum Pesarini and Sabbadini, 1998: 47

Type information: Holotype $\hat{\sigma}$, collection Carlo Pesarini and Andrea Sabbadini, Milano [type locality 'Uludağ' (Turkey: Bursa)]

Provincial distribution in Turkey: TR-A: BS (Pesarini and Sabbadini 1998, 2013; Löbl and Smetana 2010; Özdikmen 2010, 2012; Danilevsky 2015a)

Range: A: TR

*D. preissi Heyden, 1894: 86

Type information: Syntypes $2 \hat{\sigma}$ and $q$, ex collection L. von Heyden, Natur-Museum und Forschungs-Institut Senckenberg, Frankfurt am Main [type locality 'Kastamonu' (Turkey)] Provincial distribution in Turkey: TR-A: KS (Heyden 1894; Breuning 1962a; Braun 1978a; Önalp 1991; Löbl and Smetana 2010; Özdikmen 2010, 2012; Bernhauer and Peks 2011 b; Danilevsky 2015a)

Range: A: TR

*D. pseudarcivagum Breuning, 1943: 91

Type information: Holotype $\widehat{\partial}$, ex collection S. Breuning, Muséum d'Histoire Naturelle de Genève [type locality 'Amasya' (Turkey)]

brevidorsale Breuning, 1946: 109 (D. pseudarcivagum m.) [Turkey: Samsun]

Provincial distribution in Turkey: TR-A: AM SM (Breuning 1943, 1946, 1962a; Löbl and Smetana 2010; Özdikmen 2010, 2012; Danilevsky 2015a)

Range: A: TR

*D. pseudinfernale Breuning, 1943: 94

Type information: Holotype $\hat{\sigma}$, ex collection S. Breuning, Muséum d'Histoire Naturelle de Genève [type locality 'Amanos Mts.' (Turkey: Hatay)]

pseudimmutatum Breuning, 1948: 61 (D. pseudinfernale m.) [Turkey: Hatay: Amanos Mts.]

Provincial distribution in Turkey: TR-A: HT (Breuning 1943, 1948, 1962a; Löbl and Smetana 2010; Özdikmen 2010, 2012; Danilevsky 2015a)

Range: A: TR

*D. pseudobithyniense Breuning, 1962a: 465

Type information: Holotype $\hat{\sigma}$, ex collection S. Breuning, Muséum d'Histoire Naturelle de Genève [type locality 'W Balıkesir' (Turkey)]

Provincial distribution in Turkey: TR-A: BL CA (Breuning 1962a; Braun 1978a; Adlbauer 1988; Pesarini and Sabbadini 2009; Löbl and Smetana 2010; Özdikmen 2010, 2012; Danilevsky 2015a)

Range: A: TR

*D. pseudocinctellum Breuning, 1943: 90

Type information: Holotype $\hat{\sigma}$, ex collection S. Breuning, Muséum d'Histoire Naturelle de Genève [type locality 'Akşehir' (Turkey: Konya)]

fuscoplagiatum Breuning, 1946: 101 (D. pseudocinctellum m.) [Turkey: Konya: Akşehir] fuscoconjunctum Breuning, 1946: 101 (D. pseudocinctellum m.) [Turkey: Konya: Akşehir] Provincial distribution in Turkey: TR-A: KN US (Breuning 1943, 1946, 1962a; Demelt 1963; Löbl and Smetana 2010; Özdikmen 2010, 2012; Lingafelter et al. 2014; Danilevsky 2015a) Range: A: TR 
*D. pseudoholosericeum Breuning, 1962a: 300

Type information: Holotype $\widehat{\partial}$, ex collection S. Breuning, Muséum d'Histoire Naturelle de Genève [type locality 'Sinop' (Turkey)]

Provincial distribution in Turkey: TR-A: SN (Breuning 1962a; Braun 1978a; Löbl and Smetana 2010; Özdikmen 2010, 2012; Danilevsky 2015a)

Range: A: TR

*D. pseudopreissi Breuning, 1962a: 411

Type information: Holotype $\hat{\sigma}$, ex collection S. Breuning, Muséum d'Histoire Naturelle de Genève [type locality 'S Sinop' (Turkey)]

Provincial distribution in Turkey: TR-A: AM SN (Breuning 1962a; Adlbauer 1992; Löbl and Smetana 2010; Özdikmen 2010, 2012; Danilevsky 2015a)

Range: A: TR

\section{D. punctipenne Küster, 1852: 94}

Type information: Holotype, ex collection Heinrich Carl Küster > Johann Menzel, Naturhistorisches Museum, Nürnberg [type locality 'Kleinasien' (Turkey)]

distinguendum Pic, 1931c: 10 (D. olympicum var.) [Greece]

Provincial distribution in Turkey: TR-A: IS; TR-E: IS (Küster 1852; Breuning 1962a; Löbl and Smetana 2010; Özdikmen 2010, 2012; Danilevsky 2015a; Özdikmen and Kaya 2015e)

Range: E: GR TR A: TR

*D. punctulatum Pesarini and Sabbadini, 2013: 57

Type information: Holotype $\hat{\delta}$, collection Carlo Pesarini and Andrea Sabbadini, Milano [type locality 'Tepeköy' (Turkey: Manisa)]

Provincial distribution in Turkey: TR-A: MN (Pesarini and Sabbadini 2013; Danilevsky 2015a)

Range: A: TR

*D. punctulicolle Breuning, 1944: 12

Type information: Holotype $\widehat{\partial}$, ex collection S. Breuning, Muséum d'Histoire Naturelle de Genève [type locality 'Diyarbakır' (Turkey)]

Provincial distribution in Turkey: TR-A: DI (Breuning 1944, 1962a; Löbl and Smetana 2010; Özdikmen 2010, 2012; Danilevsky 2015a)

Range: A: TR

*D. pygmaeum Breuning, 1947: 168

Type information: Holotype $\widehat{\partial}$, ex collection S. Breuning, Muséum d'Histoire Naturelle de Genève [type locality 'Anatolia' (Turkey)]

presuturesignatum Breuning, 1947: 169 (D. pygmæum m.) [Turkey]

Provincial distribution in Turkey: TR-A: Kurdistan (?DI) (Breuning 1947, 1962a; Löbl and Smetana 2010; Özdikmen 2010, 2012; Danilevsky 2015a)

Range: A: TR

*D. quadripustulatum Kraatz, 1873: 88

Type information: Holotype $\uparrow$, ex collection G. Kraatz, Deutsches Entomologisches Institut, Eberswalde [type locality 'Dardanellen' (Turkey: Çanakkale)] tenuefasciatum Breuning, 1948: 60 (D. quadripustulatum m.) [Turkey: Çanakkale] 
Provincial distribution in Turkey: TR-A: CA (Kraatz 1873; Breuning 1948, 1962a; Löbl and Smetana 2010; Özdikmen 2010, 2012; Danilevsky 2015a)

Range: A: TR

D. regulare Pic, 1931b: 6 (D. gallipolitanum var.)

D. regulare regulare Pic, 1931 b: 6 (D. gallipolitanum var.)

Type information: Holotype, ex collection Dr. Buresch > M. Pic, Muséum National d'Histoire Naturelle, Paris [type locality 'Keşan' (Turkey: Edirne)]

angustesuturale Breuning, 1946: 111 (D. regulare m.) [Greece: Thrace: Mandra]

Provincial distribution in Turkey: TR-E: ED KK (Pic 1931b; Breuning 1962a; Althoff and Danilevsky 1997; Löbl and Smetana 2010; Özdikmen 2010, 2012; Danilevsky 2015a)

Range: E: GR TR

Other known subspecies: D. regulare dramaticum Pesarini and Sabbadini, 2010: 190 E: GR

*D. resadiyeense Bernhauer and Peks, $2011 \mathrm{a}: 219$

Type information: Holotype $\widehat{\sigma}$, collection Dieter Bernhauer, Bad Schwalbach [type locality 'Reşadiye' (Turkey: Malatya)]

Provincial distribution in Turkey: TR-A: MA (Bernhauer and Peks 2011a, 2013b; Danilevsky 2015a)

Range: A: TR

*D. rigattii Breuning, 1966b: 145

Type information: Holotype $\hat{\jmath}$, collection Carlo Pesarini and Andrea Sabbadini, Milano [type locality 'Zara' (Turkey: Sivas)]

atroampliatum Breuning, 1966b: 145 (D. rigattii m.) [Turkey: Sivas: Zara]

Provincial distribution in Turkey: TR-A: AM CO SV (Perissinotto and Luchini 1966; Breuning 1966b; Braun 1978a; Adlbauer 1992; Pesarini and Sabbadini 1994, 2013; Löbl and Smetana 2010; Özdikmen 2010, 2012; Danilevsky 2015a)

Range: A: TR

*D. rizeanum Breuning and Villiers, 1967: 60 (Pedestredorcadion)

Type information: Holotype $\hat{\partial}$, The Natural History Museum, London [type locality 'Sivrikaya' (Turkey: Rize)]

ikizderense Breuning, 1973: 54 [Turkey: Rize: İkizdere]

Provincial distribution in Turkey: TR-A: RI (Breuning and Villiers 1967; Breuning 1973;

Holzschuh 1977; Braun 1978a; Özdikmen 2007, 2010, 2012; Löbl and Smetana 2010;

Danilevsky 2015a)

Range: A: TR

*D. robustum Ganglbauer, 1884: 500

Type information: Holotype $\hat{\jmath}$, ex collection G. Kraatz, Deutsches Entomologisches Institut, Eberswalde [type locality 'Amasya' (Turkey)]

Provincial distribution in Turkey: TR-A: AM (Ganglbauer 1884; Breuning 1962a; Löbl and Smetana 2010; Özdikmen 2010, 2012; Danilevsky 2015a)

Range: A: TR 
*D. rolandmenradi Peks, 1992: 197

Type information: Holotype $\hat{\sigma}$, collection Heinz Peks, Schwanfeld [type locality 'Ballısaray' (Turkey: Bursa)]

Provincial distribution in Turkey: TR-A: BS KU (Peks 1992; Löbl and Smetana 2010; Özdikmen 2010, 2012; Danilevsky 2015a)

Range: A: TR

\section{D. rosti Pic, 1900c: 82}

Type information: Holotype, ex collection M. Pic, Muséum National d'Histoire Naturelle, Paris [type locality 'Caucasus']

kalinowskyi König, 1901: 10 [Georgia: Sekarpasse]

Provincial distribution in Turkey: TR-A: DI (Plavilstshikov 1958; Özdikmen and Hasbenli 2004a; Löbl and Smetana 2010; Özdikmen 2010, 2012; Danilevsky 2015a)

Range: A: GG TR

*D. rufoapicipenne Breuning, 1946: 125

Type information: Holotype [type locality 'Bayburt' (Turkey)] rufoapicale Breuning, 1943: 91 [HN] [Turkey: Bayburt] rufobrunneotomentosum Breuning, 1946: 125 (D. rufoapicenne m.) [Turkey: Bayburt]

Provincial distribution in Turkey: TR-A: AN BY DU GI RI SV (Breuning 1943, 1946, 1962a; Braun 1978a, 1979; Sama 1982; Özdikmen and Demir 2006; Löbl and Smetana 2010; Özdikmen 2010, 2012; Özdikmen et al. 2012d; Danilevsky 2015a)

Range: A: TR

*D. sabanoezueense Bernhauer and Peks, 2013b: 326

Type information: Holotype $\hat{\sigma}$, collection Dieter Bernhauer, Bad Schwalbach [type locality 'Şabanözü' (Turkey: Çankırı)]

Provincial distribution in Turkey: TR-A: CN (Bernhauer and Peks 2013b; Danilevsky 2015a) Range: A: TR

*D. sarkislaense Bernhauer and Peks, 2014a: 105

Type information: Holotype $\hat{\sigma}$, collection Dieter Bernhauer, Bad Schwalbach [type locality 'S Şarkışla' (Turkey: Sivas)]

Provincial distribution in Turkey: TR-A: SV (Bernhauer and Peks 2014a; Danilevsky 2015a) Range: A: TR

\section{D. saulcyi J. Thomson, 1865: 549}

Type information: Holotype $\hat{\sigma}$, ex collection J. Thomson > R. Oberthür, Muséum National d'Histoire Naturelle, Paris [type locality '?Syria']

simile Kraatz, 1884: 234 [Turkey: Malatya]

fenestratum Pic, 1895b: cclxxxiv [Turkey: Gaziantep ('Ain-Tab'), not Syria]

adanense Pic, 1904: 4 (D. destinoi var.) [Turkey: Adana]

bilatevittatum Pic, 1927: 167 (D. saulcyi var.) [Syria: Aleppo]

subelongatum Pic, 1931a: 1 (D. destinoi var.) [Turkey: Hatay: Akbez]

Provincial distribution in Turkey: TR-A: AD GA HT IC MA OS (Kraatz 1884; Ganglbauer 1886; Pic 1895b, 1904, 1931a; Breuning 1962a; Demelt 1963; Fuchs and Breuning 1971; Braun 1978a; Adlbauer 1992; Özdikmen et al. 2005, 2010; Löbl and Smetana 2010; Özdikmen 2010, 2012; Cihan et al. 2013; Danilevsky 2015a; Özdikmen and Kaya 2015b) Range: A: SY TR 


\section{D. scabricolle Dalman, 1817: 174 (Lamia)}

*D. scabricolle balikesirense Breuning, 1962a: 460

Type information: Holotype $\widehat{\partial}$, ex collection S. Breuning, Muséum d'Histoire Naturelle de Genève [type locality 'Balıkesir' (Turkey)]

Provincial distribution in Turkey: TR-A: BL (Breuning 1962a; Braun 1978a; Löbl and Smetana 2010; Özdikmen 2010, 2012; Danilevsky 2015a)

Range: A: TR

*D. scabricolle caramanicum K. Daniel and J. Daniel, 1903: 332

Type information: Syntypes $\hat{\sigma}$ and $\rho+$, ex collection K. Daniel, Deutsches Entomologisches Institut, Eberswalde [type locality 'Bulghar-Maaden und BulgharMagara (Bolkar Mts.-Çamardı)' (Turkey: Niğde)]

bulghardaghense Breuning, 1946: 121 (D. scabricolle m.) ['Bulghar-Maaden' (Turkey: Bolkar Mts.: Niğde: Çamardı)]

Provincial distribution in Turkey: TR-A: NI (Bodemeyer 1900; Daniel and Daniel 1903; Breuning 1946, 1962a; Löbl and Smetana 2010; Özdikmen 2010, 2012; Danilevsky 2015a) Range: A: TR

*D. scabricolle crassofasciatum Özdikmen in Küçükkaykı et al., 2013: 56

Type information: Holotype $\hat{\sigma}$, Department of Biology at Eskişehir Osmangazi University, Eskişehir [type locality 'Edremit: Sarıkız hill' (Turkey: Balıkesir)]

Provincial distribution in Turkey: TR-A: BL (Özdikmen in Küçükkaykı et al. 2013; Danilevsky 2015a)

Range: A: TR

*D. scabricolle paphlagonicum Breuning, 1962a: 459

Type information: Holotype $\widehat{\delta}$, ex collection S. Breuning, Muséum d'Histoire Naturelle de Genève [type locality 'Kastamonu' (Turkey)]

Provincial distribution in Turkey: TR-A: CN KS (Breuning 1962a; Braun 1978a; Önalp 1991; Löbl and Smetana 2010; Özdikmen 2010, 2012; Al-Hamadani and Özdikmen 2014; Danilevsky 2015a)

Range: A: TR

D. scabricolle scabricolle Dalman, 1817: 174 (Lamia)

Type information: Holotype, ex Musaeo Schönherr, Naturhistoriska Riksmuseet, Stockholm [type locality 'Georgia']

modestum Tournier, 1872: 338 [Azerbaijan: Annenfeld]

lutescens Kraatz, 1873: 50 [Caucasus]

aeruginosum Breuning, 1946: 121 (D. scabricolle m.) [Turkey: Konya: Akşehir]

Provincial distribution in Turkey: TR-A: AD AF AG AN ANT AR BI CO ER EZ GU IC IP KA KAR KN KU KY MA SV US VA YO (Heyden 1888; Bodemeyer 1900; Breuning 1946, 1962a; Demelt 1963; Perissinotto and Luchini 1966; Breuning and Villiers 1967; Fuchs and Breuning 1971; Gfeller 1972; Tuatay et al. 1972; Özbek 1978; Braun 1978a; Sama 1982; Adlbauer 1988; Önalp 1991; Özdikmen and Hasbenli 2004a; Özdikmen et al. 2005, 2009, 2010; Özdikmen and Demir 2006; Özdikmen and Okutaner 2006; Löbl and Smetana 2010; Özdikmen 2010; Turgut and Özdikmen 2010; Okutaner et al. 2011; Sama et al. 2012; Cihan et al. 2013; Şenyüz and Özdikmen 2013; Danilevsky 2015a)

Range: $A$ : $A B$ AR GG TR 
*D. scabricolle uludaghicum Breuning, 1970: 98

Type information: Holotype $\widehat{\partial}$, Muséum National d'Histoire Naturelle, Paris [type locality 'Uludağ' (Turkey: Bursa)]

Provincial distribution in Turkey: TR-A: BS (Breuning and Villiers 1967; Braun 1978a; Löbl and Smetana 2010; Özdikmen 2010, 2012; Danilevsky 2015a)

Range: A: TR

Other known subspecies: $D$. scabricolle corpulentum Ménétriés, 1832: $226 A$ : AB IN; $D$. scabricolle gilanense Lazarev, 2013: 227 A: IN; D. scabricolle elisabethpolicum Suvorov, 1915: 119 A: $A B$; D. scabricolle nakhiczevanum Danilevsky, 1999: 28 A: $A B ; D$. scabricolle paiz Danilevsky, 1999: 28 A: AB; D. scabricolle sagezense Lazarev, 2013: 227 A: IN; D. scabricolle skoupyi Lazarev, 2013: 226 A: IN; D. scabricolle sevangense Reitter, 1889: 41 A: AR; D. scabricolle subcorpulentum Breuning, 1946: 121 A: IN

*D. schultzei Heyden, 1894: 86

Type information: Syntypes $2 \hat{\partial} \widehat{\partial}$ and $q$, ex collection L. von Heyden, Natur-Museum und Forschungs-Institut Senckenberg, Frankfurt am Main [type locality 'Mardin' (Turkey)] Provincial distribution in Turkey: TR-A: MR (Heyden 1894; Breuning 1962a; Braun 1978a; Löbl and Smetana 2010; Özdikmen 2010, 2012; Danilevsky 2015a)

Range: A: TR

*D. scrobicolle Kraatz, 1873: 97

*D. scrobicolle morulum Holzschuh, 1995: 42

Type information: Holotype $\hat{\sigma}$, collection Carolus Holzschuh, Villach [type locality 'Gökdere' (Turkey: Tokat)]

fuscosubrevestitum Breuning, 1962a: 372 (D. sccrobicolle m.) [Turkey: Tokat: Niksar] Provincial distribution in Turkey: TR-A: TO (Breuning 1962a; Fuchs and Breuning 1971; Adlbauer 1992; Holzschuh 1995; Löbl and Smetana 2010; Özdikmen 2010, 2012; Danilevsky 2015a)

Range: A: TR

\section{*D. scrobicolle scrobicolle Kraatz, 1873: 97}

Type information: Syntypes $\hat{\sigma}$ and $q$, ex Musaeo Mniszech > R. Oberthür, Muséum National d'Histoire Naturelle, Paris [type locality 'Kleinasien' (Turkey)]

Provincial distribution in Turkey: TR-A: AM YO (Kraatz 1873; Ganglbauer 1884; Breuning 1962a; Fuchs and Breuning 1971; Braun 1978a; Löbl and Smetana 2010; Özdikmen 2010, 2012; Danilevsky 2015a)

Range: A: TR

*D. sebenense Bernhauer and Peks, 2011b: 202

Type information: Holotype $\widehat{\partial}$, collection Dieter Bernhauer, Bad Schwalbach [type locality 'Nallıhan' (Turkey: Ankara)]

Provincial distribution in Turkey: TR-A: AN BO (Bernhauer and Peks 2011b; Danilevsky 2015a)

Range: A: TR 
*D. semibrunneum Pic, 1903b: 170 (D. mniszechi var.)

*D. semibrunneum mediocreimpressum Pic, 1909a: 123 (D. mniszechi var.)

Type information: Holotype $q$ [type locality 'Anamas Gbg. (Dedegöl Mt.)' (Turkey: Isparta)]

medioimpressum Pic, 1910: 5 (D. semibrunneum var.) [wrong posterior spelling unavailable]

anamasum Pic, 1934: 33 (D. mniszechi ssp.) [Turkey: Isparta: Dedegöl Mt.]

Provincial distribution in Turkey: TR-A: AF IP (Pic 1909a, 1934; Breuning 1962a; Löbl and

Smetana 2010; Özdikmen 2010, 2012; Pesarini and Sabbadini 2013; Lazarev 2014; Danilevsky 2015a)

Range: A: TR

*D. semibrunneum notatum Pesarini and Sabbadini, 2013: 62

Type information: Holotype $\hat{\sigma}$, collection Carlo Pesarini and Andrea Sabbadini, Milano [type locality 'Bala' (Turkey: Ankara)]

Provincial distribution in Turkey: TR-A: AN (Pesarini and Sabbadini 2013; Danilevsky 2015a) Range: A: TR

*D. semibrunneum semibrunneum Pic, 1903b: 170 (D. mniszechi var.)

Type information: Syntype, ex collection M. Pic, Muséum National d'Histoire Naturelle, Paris [type locality 'Bozdağ' (Turkey: İzmir)]

boszdaghana Breuning, 1948: 61 (D. mniszechi ssp. semibrunneum m.) [Turkey: İzmir:

Bozdağ]

Provincial distribution in Turkey: TR-A: ES IZ (Pic 1903b; Bodemeyer 1906; Breuning 1948, 1962a; Löbl and Smetana 2010; Özdikmen 2010, 2012; Pesarini and Sabbadini 2013; Danilevsky 2015a)

Range: A: TR

*D. semilineatum Fairmaire, 1866: 273

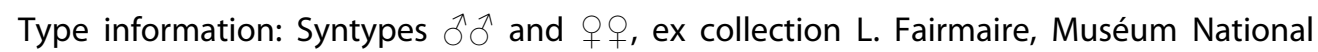
d'Histoire Naturelle, Paris [type locality 'Bozdağ' (Turkey: İzmir)]

medioincisum Breuning, 1946: 108 (D. semilineatum m.) [Turkey: İzmir: Bozdağ]

Provincial distribution in Turkey: TR-A: IZ KN (Fairmaire 1866; Ganglbauer 1884; Breuning 1946, 1962a; Tuatay et al. 1972; Özdikmen et al. 2005; Löbl and Smetana 2010; Özdikmen 2010, 2012; Danilevsky 2015a)

Range: A: TR

*D. semivelutinum Kraatz, 1873: 82

Type information: Holotype + , ex Musaeo Mniszech > R. Oberthür, Muséum National d'Histoire Naturelle, Paris [type locality 'Kleinasien' (Turkey)]

Provincial distribution in Turkey: TR-A: SV (Kraatz 1873; Breuning 1962a; Löbl and Smetana 2010; Özdikmen 2010, 2012; Rapuzzi and Sama 2012; Danilevsky 2015a)

Range: A: TR

D. septemlineatum Waltl, 1838: 469

*D. septemlineatum abanti Braun, 1976c: 54

Type information: Holotype $\hat{\sigma}$, collection Walter Braun, Tübingen [type locality 'Abant' (Turkey: Bolu)] 
Provincial distribution in Turkey: TR-A: AN BO CN DU KR (Braun 1976c, 1978a; Sama 1982; Önalp 1991; Adlbauer 1992; Özdikmen and Hasbenli 2004a; Özdikmen et al. 2005, 2012d; Özdikmen 2007, 2010, 2012; Löbl and Smetana 2010; Al-Hamadani and Özdikmen 2014; Danilevsky 2015a)

Range: A: TR

*D. septemlineatum demirciense Breuning, 1966a: 19

Type information: Holotype $\hat{\delta}$, Institut Royal des Sciences Naturelles de Belgique [type locality 'Demirci' (Turkey: ?Manisa)]

subapicale Breuning, 1966a: 20 (D. septemlineatum ssp. demirciense m.) [Turkey: ? Manisa: Demirci]

Provincial distribution in Turkey: TR-A: MN (Breuning 1966a; Löbl and Smetana 2010; Özdikmen 2010, 2012; Danilevsky 2015a)

Range: A: TR

*D. septemlineatum novemlineatum Kraatz, 1873: 61

Type information: [type locality 'Bilecik, Eskişehir' (Turkey)]

forcipiferum Kraatz, 1873: 60 ['Palestina', undoubtedly mislabeled]

dorsobasireductum Breuning, 1946: 106 (D. septemlineatum ssp. novemlineatum m.)

[Turkey: Eskişehir]

Provincial distribution in Turkey: TR-A: AF BI BU ES GA IP KN KU (Bodemeyer 1900; Breuning 1946, 1962a; Braun 1978a; Adlbauer 1988; Önalp 1991; Özdikmen et al. 2005; Löbl and Smetana 2010; Özdikmen 2010, 2012; Şenyüz and Özdikmen 2013; Danilevsky 2015a)

Range: A: TR

*D. septemlineatum octolineatum Kraatz, 1873: 61

Type information: [type locality 'Bursa' (Turkey)]

pseudapiceconjunctum Breuning, 1946: 105 (D. septemlineatum ssp. octolineatum m.)

[Turkey: Bursa]

Provincial distribution in Turkey: TR-A: BL BS CA IS KO SA (Heyden 1884; Breuning 1946, 1962a; Fuchs and Breuning 1971; Braun 1978a; Adlbauer 1988; Önalp 1991; Löbl and Smetana 2010; Özdikmen 2010, 2012; Tekin and Özdikmen 2015; Danilevsky 2015a)

Range: A: TR

D. septemlineatum septemlineatum Waltl, 1838: 469

Type information: Syntypes, ex collection Ménétriés > Joseph Waltl, Naturhistorisches Museum Wien [type locality 'Turkei' (Turkey)] apicale J. Thomson, 1865: 549 [Turkey] rufoapicale Pic, 1942a: 2 (D. septemlineatum var.) [Greece: Salonica] basilineatum Breuning, 1946: 105 (D. septemlineatum septemlineatum m.) [Turkey] Provincial distribution in Turkey: TR-E: IS (Küster 1846b; Kraatz 1873; Breuning 1946, 1962a; Althoff and Danilevsky 1997; Löbl and Smetana 2010; Özdikmen 2010, 2012; Danilevsky 2015a)

Range: E: BU GR TR

*D. sinopense Breuning, 1962a: 299

Type information: Holotype $\widehat{\partial}$, ex collection S. Breuning, Muséum d'Histoire Naturelle de Genève [type locality 'Bergen' (Turkey: S Sinop)] 
reductevittipenne Breuning, 1962a: 300 (D. sinopense m.) [Turkey: S Sinop: Bergen] drannadaghense Breuning, 1970: 98 (D. sinopense m.) [Turkey: Sinop: Dranaz Mt.] nigrovittipenne Breuning, 1974b: 65 (D. sinopense m.) [Turkey: Sinop: Dranaz Mt.] Provincial distribution in Turkey: TR-A: SN (Breuning 1962a, 1970, 1974b; Braun 1978a; Löbl and Smetana 2010; Özdikmen 2010, 2012; Danilevsky 2015a)

Range: A: TR

\section{*D. sinuatevittatum Pic, 1937: 6}

Type information: Holotype $\hat{\partial}$, ex collection Heyrovský > M. Pic, Muséum National d'Histoire Naturelle, Paris [type locality 'Taurus: Salbakos Mt. (Babadağ)' (Turkey: Denizli)] brevemediolineatum Pic, 1942b: 6 [Turkey: Taurus Mt.]

Provincial distribution in Turkey: TR-A: BU DE KA (Pic 1937, 1942b; Breuning 1962a; Özdikmen et al. 2005; Özdikmen and Okutaner 2006; Löbl and Smetana 2010; Özdikmen 2010, 2012; Danilevsky 2015a)

Range: A: TR

*D. skoupyorum Bernhauer and Peks, 2014b: 191 [RN]

Type information: Holotype $\hat{\sigma}$, collection Heinz Peks, Schwanfeld as D. skoupyi [type locality 'Şavşat and Çam pass' (Turkey: Artvin)]

skoupyi Bernhauer and Peks, 2013b: 326 [HN]

Provincial distribution in Turkey: TR-A: ART (Bernhauer and Peks 2013b, 2014b; Danilevsky 2015a)

Range: A: TR

D. sodale Hampe, 1852: 313

*D. sodale blumenthali Breuning, 1966a: 20

Type information: Holotype $\widehat{\partial}$, Institut Royal des Sciences Naturelles de Belgique [type locality 'Giresun' (Turkey)]

Provincial distribution in Turkey: TR-A: Gl (Breuning 1966a; Löbl and Smetana 2010; Özdikmen 2010, 2012; Danilevsky 2015a)

Range: A: TR

D. sodale sodale Hampe, 1852: 313

Type information: Syntypes, ex collection Moritz Wagner, Museum für Naturkunde der Humboldt-Universität zu Berlin) [type locality 'Kurdistan' (Turkey)]

Provincial distribution in Turkey: TR-A: EZ (Plavilstshikov 1958; Gfeller 1972; Braun 1978a; Önalp 1990; Löbl and Smetana 2010; Özdikmen 2010, 2012; Danilevsky 2015a) Range: A: ?GG TR

*D. sodale trapesunticum Breuning, 1946: 124

Type information: Holotype $\hat{\sigma}$, ex collection S. Breuning, Muséum d'Histoire Naturelle de Genève [type locality 'Trabzon' (Turkey)]

Provincial distribution in Turkey: TR-A: BY TB (Breuning 1946, 1962a; Villiers 1959; Breuning and Villiers 1967; Demelt 1967; Adlbauer 1988; Löbl and Smetana 2010; Özdikmen 2010, 2012; Danilevsky 2015a)

Range: A: TR 
*D. sonjae Peks, 1993: 5

Type information: Holotype $\hat{\sigma}$, collection Heinz Peks, Schwanfeld [type locality 'Elmapınarı' (Turkey: Elazığ)]

Provincial distribution in Turkey: TR-A: EL (Peks 1993; Pesarini and Sabbadini 2004; Löbl and Smetana 2010; Özdikmen 2010, 2012; Danilevsky 2015a)

Range: A: TR

*D. steineri Holzschuh, 1977: 129

Type information: Holotype $\widehat{\partial}$, collection Carolus Holzschuh, Villach [type locality 'Çamlıhemşin: Hacı Vanak plateau' (Turkey: Rize)]

Provincial distribution in Turkey: TR-A: RI (Holzschuh 1977; Löbl and Smetana 2010; Özdikmen 2010, 2012; Danilevsky 2015a)

Range: A: TR

\section{D. striolatum Kraatz, 1873: 93}

Type information: Syntypes $\widehat{\partial} \hat{\sigma}$ and $q+$, ex collection G. Kraatz, Deutsches Entomologisches Institut, Eberswalde [type locality 'Caucasus'] obesum Tournier, 1872: 340 [HN] [Georgia: Surami] masculinum Plavilstshikov, 1927: 51 [RN] (D. striolatum ab.) [Georgia: Surami] suramense Breuning, 1946: 98 [RN] (D. striolatum ssp.) [Georgia: Surami]

Provincial distribution in Turkey: TR-A: AG (Kraatz 1873; Plavilstshikov 1958; Villiers 1967; Önalp 1991; Löbl and Smetana 2010; Özdikmen 2010; Danilevsky 2015a)

Range: A: ?AB AR GG ?IN TR

\section{D. sturmii Frivaldszky von Frivald, 1837: 179}

Type information: Syntypes, ex collection Imre Frivaldszky, Magyar Természettudományi Mûzeum, Budapest [type locality 'Türkei' (Turkey)]

Provincial distribution in Turkey: TR-E: ED (Frivaldszky von Frivald 1837; Waltl 1838; Thomson 1867; Önalp 1991; Özdikmen 2010; Danilevsky 2015a) Range: E: BU MC TR

*D. subatritarse Breuning, 1966b: 146

Type information: Holotype $\hat{\sigma}$, collection Antonello Perissinotto [type locality 'Boğazkale' (Turkey: Çorum)]

Provincial distribution in Turkey: TR-A: CO KIR (Perissinotto and Luchini 1966; Breuning 1966b; Braun 1978a; Özdikmen 2010, 2012; Özdikmen and Kaya 2015a)

Range: A: TR

*D. subcinctellum Breuning, 1962a: 275

Type information: Holotype $\widehat{\partial}$, ex collection Pierre Lepesme, Musée d'Histoire Naturelle de Lyon [type locality 'Yazıhan: Hamidiye' (Turkey: Malatya)]

Provincial distribution in Turkey: TR-A: MA (Breuning 1962a; Löbl and Smetana 2010; Özdikmen 2010, 2012; Danilevsky 2015a)

Range: A: TR

\section{*D. subinterruptum Pic, 1900a: 12 (D. divisum var.)}

Type information: Holotype, ex collection M. Pic, Muséum National d'Histoire Naturelle, Paris [type locality 'Konya' (Turkey)] 
indivisum Pic, 1900a: 12 (D. divisum var.) [Turkey: Konya] interruptevittatum Breuning, 1946: 106 (D. divisum m.) [Turkey: Afyonkarahisar] exiguum Breuning, 1946: 108 (D. divisum m.) [Turkey: Konya] posticeconjunctum Breuning, 1946: 108 (D. divisum m.) [Turkey: Konya] Provincial distribution in Turkey: TR-A: AF ES IZ ?IC KN; TR-E: ED (Pic 1900a; Breuning 1946, 1962a; Braun 1978a; Löbl and Smetana 2010; Özdikmen 2010, 2012; Sama et al. 2010; Danilevsky 2015a)

Range: E: TR A: TR

*D. subsericatum Pic, 1901: 12

*D. subsericatum majus Breuning, 1962a: 371 (D. rufipenne ssp. major)

Type information: Holotype $\hat{\jmath}$, ex collection S. Breuning, Muséum d'Histoire Naturelle de Genève [type locality 'Sinop' (Turkey)]

Provincial distribution in Turkey: TR-A: SN (Breuning 1962a; Braun 1978a; Sama 1982;

Pesarini and Sabbadini 1998; Löbl and Smetana 2010; Özdikmen 2010, 2012; Danilevsky 2015a)

Range: A: TR

*D. subsericatum rufipenne Breuning, 1946: 118 (D. rufipenne)

Type information: Holotype $\hat{\delta}$, ex collection Vogt [type locality 'llgaz Mt.' (Turkey: Kastamonu-Çankırı)]

cankiriense Breuning, 1962b: 39 (D. rufipenne m.) [Turkey: Çankırı]

antenigrescens Breuning, 1962b: 39 (D. rufipenne ssp. cankiriense m.) [Turkey: Çankırı] univittatum Breuning, 1962a: 371 (D. rufipenne m.) [Turkey: Kastamonu: Ilgaz Mt.]

multivittipenne Breuning, 1962a: 371 (D. rufipenne m.) [Turkey: Kastamonu: Ilgaz Mt.] Provincial distribution in Turkey: TR-A: AN CN KS (Breuning 1946, 1962a; Demelt 1967; Braun 1978a; Sama 1982; Adlbauer 1988, 1992; Pesarini and Sabbadini 1998; Özdikmen 2006, 2010, 2012; Löbl and Smetana 2010; Al-Hamadani and Özdikmen 2014; Danilevsky 2015a)

Range: A: TR

*D. subsericatum subsericatum Pic, 1901: 12

Type information: Holotype, ex collection Théry > M. Pic, Muséum National d'Histoire Naturelle, Paris [type locality 'Syria' but undoubtedly mislabeled, should be Turkey]

paphlagoniense Breuning, 1944: 12 [Turkey: Paphlagonia: Kastamonu]

Provincial distribution in Turkey: TR-A: KS (Pic 1901; Breuning 1944, 1962a; Braun 1978a; Löbl and Smetana 2010; Özdikmen 2010, 2012; Danilevsky 2015a)

Range: A: TR

*D. subsericatum vulneratum Pesarini and Sabbadini, 1998: 51

Type information: Holotype $\hat{\sigma}$, collection Carlo Pesarini and Andrea Sabbadini, Milano [type locality 'Masruf pass' (Turkey: Kastamonu)]

Provincial distribution in Turkey: TR-A: KS (Pesarini and Sabbadini 1998; Löbl and Smetana 2010; Özdikmen 2010, 2012; Danilevsky 2015a)

Range: A: TR

*D. subvestitum K. Daniel, 1900: 140

Type information: Syntypes $\hat{\partial}$ and $q++$, ex collection K. Daniel, Deutsches Entomologisches Institut, Eberswalde [type locality 'Bozdağ' (Turkey: İzmir)] 
Provincial distribution in Turkey: TR-A: AM AN ES IZ KN MA NI (Daniel 1900; Breuning 1962a; Gfeller 1972; Önalp 1990; Löbl and Smetana 2010; Özdikmen 2010, 2012; Danilevsky 2015a)

Range: A: TR

\section{D. sulcipenne Küster, 1847: 87}

D. sulcipenne argonauta Suvorov, 1913: 74 (D. argonauta)

Type information: Syntypes $\widehat{\partial} \hat{\sigma}$ and $q q$, ex collection G. L. Suvorov, Zoological Museum, University of Moscow [type locality 'Erivan' (Armenia)]

kurda Suvorov, 1913: 76 [Turkey: Kars: Sarıkamış]

Provincial distribution in Turkey: TR-A: EZ KAR (Suvorov 1913; Plavilstshikov 1958; Löbl and Smetana 2010; Özdikmen 2010; Danilevsky 2015a)

Range: A: AR GG TR

Other known subspecies: D. sulcipenne plyushchi Danilevsky, 2014: 673 A: GG; D. sulcipenne gubini Danilevsky, 2014: 674 A: GG; D. sulcipenne borzhomiense Danilevsky, 2014: 677 A: GG; D. sulcipenne zekariense Danilevsky, 2014: 678 A: GG; D. sulcipenne paki Danilevsky, 2014: 674 A: GG; D. sulcipenne demokidovi Suvorov, 1915: 115 A: GG; D. sulcipenne caucasicum Küster, 1847: 98 A: AR GG; D. sulcipenne goktshanum Suvorov, 1915: 117 A: AR; D. sulcipenne sulcipenne Küster, 1847: 87 A: GG

*D. syriense Breuning, 1943: 94

Type information: Holotype $q$, ex collection S. Breuning, Muséum d'Histoire Naturelle de Genève [type locality 'Syria' but undoubtedly mislabeled, should be Amanos Mts. (Turkey)] Provincial distribution in Turkey: TR-A: ?HT (Breuning 1943, 1962a; Özdikmen 2010, 2012; Danilevsky 2015a)

Range: A: TR

D. tauricum Waltl, 1838: 468

D. tauricum pittinoi Pesarini and Sabbadini, 2010: 185

Type information: Holotype $\hat{\sigma}$, Museo Civico di Storia Naturale, Milano [type locality 'Doxa' (Greece)]

Provincial distribution in Turkey: TR-E: ED (Pesarini and Sabbadini 2010; Danilevsky 2015a)

Range: E: GR TR

\section{D. tauricum tauricum Waltl, 1838: 468}

Type information: Syntypes, ex collection Imre Frivaldszky, Magyar Természettudományi Mûzeum, Budapest [type locality 'Türkei' (Turkey)]

nigritarse Kraatz, 1873: 77 [?]

Provincial distribution in Turkey: TR-A: IS SA; TR-E: ED TE (Waltl 1838; Thomson 1867;

Breuning 1962a; Önalp 1991; Pesarini and Sabbadini 2010; Özdikmen 2010; Löbl and Smetana 2010; Danilevsky 2015a)

Range: E: BU GR MD RO TR UK A: TR

*D. theophilei Pic, 1898: 57

Type information: Holotype $\hat{\delta}$, ex collection M. Pic, Muséum National d'Histoire Naturelle, Paris [type locality 'Trabzon' (Turkey)] 
costiferum Pic, 1898: 57 (D. theophilei var.) [Turkey: Trabzon]

Provincial distribution in Turkey: TR-A: ART GU TB (Pic 1898; Plavilstshikov 1958; Villiers 1959; Breuning 1962a; Löbl and Smetana 2010; Özdikmen 2010, 2012; Danilevsky 2015a) Range: A: TR

*D. turcicum Breuning, 1963: 35

Type information: Holotype $\hat{\sigma}$, ex collection Hauser, Naturhistorisches Museum Wien [type locality 'Paşalimanı' (Turkey: Balıkesir)]

Provincial distribution in Turkey: TR-A: BL (Breuning 1963; Löbl and Smetana 2010; Özdikmen 2010, 2012; Danilevsky 2015a)

Range: A: TR

*D. ullrichi Bernhauer, 1988: 102

Type information: Holotype $\hat{\sigma}$, collection Dieter Bernhauer, Bad Schwalbach [type locality 'Malatya' (Turkey)]

Provincial distribution in Turkey: TR-A: MA (Bernhauer 1988; Löbl and Smetana 2010; Özdikmen 2010, 2012; Pesarini and Sabbadini 2013; Danilevsky 2015a)

Range: A: TR

\section{D. urmianum Plavilstshikov, 1937: 27}

Type information: Holotype $\hat{\delta}$, ex collection N. N. Plavilstshikov, Zoological Museum, University of Moscow [type locality 'Urmia' (Iran)]

Provincial distribution in Turkey: TR-A: HA (Braun 1975, 1978a; Löbl and Smetana 2010; Özdikmen 2010; Sama et al. 2012; Danilevsky 2015a)

Range: A: IN TR

\section{D. variegatum Ganglbauer, 1884: 502}

Type information: Syntypes $\widehat{\partial}$ and + , ex collection G. Kraatz, Deutsches Entomologisches Institut, Eberswalde [type locality 'Syria']

apicedisjunctum Breuning, 1946: 126 (D. variegatum m.) ['Ain-Tab', Turkey: Gaziantep] nubilatum Breuning, 1946: 126 (D. variegatum m.) ['Ain-Tab', Turkey: Gaziantep]

Provincial distribution in Turkey: TR-A: GA KA (Breuning 1946, 1962a; Löbl and Smetana 2010; Özdikmen 2010; Danilevsky 2015a)

Range: A: SY TR

\section{D. wagneri Küster, 1846a: 87}

Type information: Holotype, ex collection Heinrich Carl Küster > Johann Menzel, Naturhistorisches Museum, Nürnberg [type locality 'Ararat Mt.' (Armenia)] solyzinosum Pic, 1942b: 2 [Solyzino]

Provincial distribution in Turkey: TR-A: AG EZ KAR (Kraatz 1873; Ganglbauer 1884; Plavilstshikov 1958; Önalp 1991; Löbl and Smetana 2010; Özdikmen 2010, 2012; Danilevsky 2015a)

Range: A: AR GG TR

*D. weyersii Fairmaire, 1866: 271

Type information: Syntypes $\hat{\partial} \hat{o}$ and $q+q$, ex collection L. Fairmaire, Muséum National d'Histoire Naturelle, Paris [type locality 'Kisilgye-Aole (?Ödemiş: Kızılca köyü)' (Turkey: ?ìzmir)] aolense Pic, 1942c: 1 (D. weyersi var.) [Turkey] brunneicolor Breuning, 1946: 121 (D. weyersi m.) [Turkey: İzmir: Ödemiş] subbrunneicolor Breuning, 1946: 121 (D. weyersi m.) [Turkey: İzmir: Ödemiş] 
Provincial distribution in Turkey: TR-A: BU IZ MN (Fairmaire 1866; Ganglbauer 1884; Pic 1942c; Breuning 1946, 1962a; Gül-Zümreoğlu 1972; Adlbauer 1988; Önalp 1991; Löbl and Smetana 2010; Özdikmen 2010, 2012; Danilevsky 2015a)

Range: A: TR

*D. yahyaliense Bernhauer and Peks, 2011a: 217

Type information: Holotype $\widehat{\partial}$, collection Dieter Bernhauer, Bad Schwalbach [type locality 'Yahyalı' (Turkey: Kayseri)]

Provincial distribution in Turkey: TR-A: KY (Bernhauer and Peks 2011a; Danilevsky 2015a) Range: A: TR

Subgenus D. (Maculatodorcadion) Breuning, 1943: 525

[type species Dorcadion quadrimaculatum Küster, 1848]

*D. janssensi Breuning, 1966a: 21

Type information: Holotype $\hat{\sigma}$, Institut Royal des Sciences Naturelles de Belgique [type locality 'Tatos Mts.' (Turkey: Rize-Erzurum)]

Provincial distribution in Turkey: TR-A: EZ RI (Breuning 1966a; Löbl and Smetana 2010; Özdikmen 2010, 2012; Danilevsky 2015a)

Range: A: TR

*D. phrygicum Peks, 1993: 8

Type information: Holotype $\hat{\sigma}$, collection Heinz Peks, Schwanfeld [type locality 'Kaş: Ova' (Turkey: Antalya)]

Provincial distribution in Turkey: TR-A: ANT BS CA DU (Breuning 1962a; Peks 1993; Löbl and Smetana 2010; Özdikmen 2010, 2012; Özdikmen et al. 2012d; Danilevsky 2015a; Özdikmen and Kaya 2015f)

Range: A: TR

\section{D. quadrimaculatum Küster, 1848: 79}

Type information: Syntypes $\widehat{\partial}$ and $q \propto$, , ex collection Heinrich Carl Küster $>$ Johann Menzel, Naturhistorisches Museum, Nürnberg [type locality 'Türkei' (Turkey)]

bimaculatum Pic, 1898: 56 (D. quadrimaculatum var.) [Greece: Attica] innotatum Pic, 1908: 2 (D. quadrimaculatum var.) [Greece]

clementinum Pic, 1918: 11 (D. quadrimaculatum var.) [Greece: Clementi]

Provincial distribution in Turkey: TR-E (Küster 1848; Ganglbauer 1884; Breuning 1962a; Krätschmer 1985; Althoff and Danilevsky 1997; Löbl and Smetana 2010; Özdikmen 2010, 2012; Danilevsky 2015a)

Range: E: GR TR

*D. triste Frivaldszky von Frivald, 1845: 184

Type information: Syntypes $\hat{\jmath} \hat{o}$ and $q++$, ex collection Imre Frivaldszky, Magyar Természettudományi Mûzeum, Budapest [type locality 'Smyrna' (Turkey: İzmir)]

vittipenne Breuning, 1946: 97 (D. triste m.) [Turkey: İzmir]

postmedioreductum Breuning, 1946: 97 (D. triste m.) [Turkey: İzmir]

Provincial distribution in Turkey: TR-A: BL BS IZ (Frivaldszky von Frivald 1845; Ganglbauer 1884; Breuning 1946, 1962a; Demelt 1963; Gül-Zümreoğlu 1972; Braun 1978a; Krätschmer 1985; Önalp 1990; Peks 1993; Löbl and Smetana 2010; Özdikmen 2010, 2012; Danilevsky 2015a; Özdikmen and Kaya 2015f) 
Range: A: TR

*D. wolfi Krätschmer, 1985: 24

Type information: Holotype $\widehat{\partial}$, collection O.E. Krätschmer [type locality 'Burhaniye: Ören' (Turkey: Balıkesir)]

Provincial distribution in Turkey: TR-A: BL (Krätschmer 1985; Adlbauer 1992; Löbl and Smetana 2010; Özdikmen 2010, 2012; Danilevsky 2015a)

Range: A: TR

Genus Megalodorcadion Pesarini and Sabbadini, 1998: 58

[type species Dorcadion ledereri J. Thomson, 1865]

Subgenus M. (Anatolodorcadion) Özdikmen and Kaya, 2015g: 3

[type species Dorcadion dombilicoides Özdikmen and Kaya, 2013]

*M. dombilicoides Özdikmen and Kaya, 2013: 494 (Dorcadion)

Type information: Holotype $\hat{O}$, collection H. Özdikmen, Zoology Museum of Gazi University, Ankara [type locality 'Gölünyazı' (Turkey: Çorum)]

Provincial distribution in Turkey: TR-A: CO (Özdikmen and Kaya 2013, 2015g; Danilevsky 2015a)

Range: A: TR

*M. glabrofasciatum K. Daniel, 1900: 140 (Dorcadion)

Type information: Holotype $\hat{\sigma}$, ex collection K. Daniel, Deutsches Entomologisches Institut, Eberswalde [type locality 'Bilecik' (Turkey)]

imparivittatum K. Daniel, 1900: 140 (D. glabrofasciatum var.) [Turkey]

parivittatum Breuning, 1946: 98 (D. glabrofasciatum m.) [Turkey: İzmir: Bozdağ]

glabroseparatum Breuning, 1962a: 310 (D. glabrofasciatum m.) [Turkey: Eskişehir]

Provincial distribution in Turkey: TR-A: AF BI ES IZ US (K. Bodemeyer 1900, 1906; Daniel 1900; Breuning 1946, 1962a; Demelt 1963; Braun 1978a; Önalp 1991; Pesarini and Sabbadini 2004; Löbl and Smetana 2010; Özdikmen 2010, 2012; Özdikmen and Kaya 2013, 2015g; Danilevsky 2015a)

Range: A: TR

*M. nudefasciatum Özdikmen and Şenyüz, 2015: 647

Type information: Holotype $\hat{\sigma}^{\lambda}$, collection Y. Şenyüz, Dumlupınar University, Kütahya [type locality 'Dumlupınar University' (Turkey: Kütahya)]

Provincial distribution in Turkey: TR-A: KU (Özdikmen and Şenyüz 2015)

Range: A: TR

Subgenus M. (Fusodorcadion) Özdikmen and Kaya, 2015g: 3

[type species Dorcadion parallelum Küster, 1847]

*M. parallelum Küster, 1847: 79 (Dorcadion)

Type information: Holotype $\widehat{\partial}$, ex collection Jacob Sturm, Zoologische Staatssammlung des Bayerischen Staates, München [type locality 'Türkei' (Turkey)]

rufinimembre Breuning, 1946: 132 (D. parallelum m.) [?Syria, should be Turkey]

Provincial distribution in Turkey: TR-A: AM AN CO TO YO (Küster 1847; Ganglbauer 1884; Braun 1978a; Önalp 1991; Adlbauer 1992; Özdikmen and Hasbenli 2004a; Löbl and 
Smetana 2010; Özdikmen 2010, 2012; Özdikmen and Kaya 2013, 2015g; Danilevsky 2015a)

Range: A: TR

Subgenus M. (Megalodorcadion) Pesarini and Sabbadini, 1998: 58

[type species Dorcadion ledereri J. Thomson, 1865]

*M. escherichi Ganglbauer, 1897: 54 (Dorcadion)

Type information: Syntypes $\hat{\partial} \hat{\sigma}$ and $q$, ex collection K. L. Escherich, Deutsches Entomologisches Institut, Eberswalde [type locality 'Ankara' (Turkey)]

egregium Ganglbauer, 1897: 56 [Turkey: Ankara]

angorense Ganglbauer, 1897: 57 [Turkey: Ankara]

obliquesignatum Pic, 1900a: 12 (D. escherichi var.) [Turkey: Konya]

posticedisjunctum Pic, 1909b: 99 (D. escherichi var.) [Turkey: Ankara]

Provincial distribution in Turkey: TR-A: AM AN BI KN TO (Ganglbauer 1897; Bodemeyer 1900; Pic 1900a, 1909b; Breuning 1962a; Braun 1978a; Önalp 1991; Pesarini and Sabbadini 1998; Löbl and Smetana 2010; Özdikmen 2010, 2012; Özdikmen and Kaya 2013, 2015g; Danilevsky 2015a)

Range: A: TR

*M. ledereri J. Thomson, 1865: 548 (Dorcadion)

Type information: Syntypes, ex collection J. Thomson > R. Oberthür, Muséum National d'Histoire Naturelle, Paris [type locality 'Turkey']

cappadocicum Breuning, 1946: 132 (D. ledereri m.) [Turkey: Cappadocia]

discoseparatum Breuning, 1946: 132 (D. ledereri m.) [Turkey: Amasya]

preconjunctum Breuning, 1946: 132 (D. ledereri m.) [Turkey: Amasya]

presuturenigrum Breuning, 1970: 98 (D. ledereri m.) [Turkey: Tokat]

Provincial distribution in Turkey: TR-A: AM CO SM TO (Thomson 1865, 1867; Ganglbauer 1884; Breuning 1946, 1962a, 1970; Pesarini and Sabbadini 1998; Löbl and Smetana 2010; Özdikmen 2010, 2012; Özdikmen and Kaya 2013, 2015g; Danilevsky 2015a)

Range: A: TR

*M. walteri Holzschuh, 1991: 55 (Dorcadion)

Type information: Holotype $\hat{\sigma}$, collection Carolus Holzschuh, Villach [type locality 'Alput Bey village' (Turkey: Bolu)]

Provincial distribution in Turkey: TR-A: BO (Holzschuh 1991; Pesarini and Sabbadini 1998; Löbl and Smetana 2010; Özdikmen 2010, 2012; Anichtchenko and Shavrin 2013; Özdikmen and Kaya 2013, 2015g; Danilevsky 2015a)

Range: A: TR

Genus Neodorcadion Ganglbauer, 1884: 437

[type species Lamia bilineata Germar, 1824]

Subgenus N. (Neodorcadion) Ganglbauer, 1884: 437

[type species Lamia bilineata Germar, 1824]

N. bilineatum Germar, 1824: 485 (Lamia)

Type information: Syntypes, ex collection Germar, Museum für Naturkunde der Humboldt-Universität zu Berlin [type locality 'Austria'] macedonicum Pic, 1917b: 5 (N. bilineatum var.) [Macedonia] 
Provincial distribution in Turkey: TR-E: ED IS TE (Heyden 1884; Ganglbauer 1884; Plavilstshikov 1958; Breuning and Villiers 1967; Braun 1978a; Althoff and Danilevsky 1997; Özdikmen 2010; Danilevsky 2015a; Özdikmen and Kaya 2015g)

Range: E: AL BH BU CR GR HU MC MD RO SK TR UK YU

*N. exornatoides Breuning, 1962a: 68

Type information: Holotype $\widehat{\partial}$, ex collection S. Breuning, Muséum d'Histoire Naturelle de Genève [type locality 'Balıkesir' (Turkey)]

Provincial distribution in Turkey: TR-A: BL (Breuning 1962a; Pesarini and Sabbadini 2008; Löbl and Smetana 2010; Özdikmen 2010, 2012; Danilevsky 2015a; Özdikmen and Kaya 2015g)

Range: A: TR

N. exornatum Frivaldszky von Frivald, 1835: 268 (Dorcadion)

Type information: Holotype, ex collection Imre Frivaldszky, Magyar Természettudományi Mûzeum, Budapest [type locality 'Balkans']

labyrinthicum J. Thomson, 1865: 549 (Dorcadion) [Ukraine: Crimea]

balcanicum Tournier, 1872: 284 (Dorcadion) [?Balkans 'Orient']

anticejunctum Pic, 1917b: 6 (N. balcanicum var.) [Greece: Salonica]

subdiversum Pic, 1917b: 6 (N. balcanicum var.) [Balkans]

basiseparatum Breuning, 1947: 170 (N. exornatum m.) [Turkey]

latefasciatum Breuning, 1948: 58 (N. exornatum m.) [Turkey: İstanbul: Alem Mt.]

Provincial distribution in Turkey: TR-A: IS; TR-E (Ganglbauer 1884; Breuning 1947, 1948, 1962a; Althoff and Danilevsky 1997; Pesarini and Sabbadini 2008; Löbl and Smetana 2010; Özdikmen 2010; Danilevsky 2015a; Özdikmen and Kaya 2015g)

Range: E: BU GR RO TR A: TR

\section{N. Iaqueatum Waltl, 1838: 469 (Dorcadion)}

Type information: Holotype, ex collection Imre Frivaldszky > Joseph Waltl, Naturhistorisches Museum Wien [type locality 'istanbul' (Turkey)] interruptum Mulsant and Rey, 1863: 150 (Dorcadion) [Turkey: İstanbul] sparsum Mulsant and Rey, 1863: 152 (Dorcadion) [Turkey: İstanbul] abeillei Tournier, 1872: 208 (Dorcadion) [Turkey] anceyi Pic, 1913: 186 (N. laqueatum var.) [Turkey: İstanbul] bulgaricum Pic, 1913: 186 (N. laqueatum var.) [Bulgaria] insparsum Pic, 1914: 8 (N. laqueatum var.) [Turkey: İstanbul] lemoulti Pic, 1914: 8 (N. laqueatum var.) [Turkey: İstanbul] diversesparsum Pic, 1914: 8 (N. laqueatum var.) [Turkey: İstanbul] sparsenotatum Pic, 1914: 8 (N. laqueatum var.) [Turkey: İstanbul] subinterruptum Pic, 1917b: 6 (N. laqueatum var.) [Turkey] servilorum Pic, 1917b: 6 (N. laqueatum var.) [Turkey: İstanbul] kadiense Pic, 1917a: 10 (N. balcanicum var.) ['Kadi Kei', Turkey: İstanbul: Kadıköy] rufobrunneum Breuning, 1947: 170 (N. fallax m.) [Turkey: İstanbul: Alem Mt.] nigrimembre Breuning, 1947: 170 ( $N$. laqueatum m.) [Turkey: İstanbul: Alem Mt.] Provincial distribution in Turkey: TR-A: AM BS IS KO; TR-E: IS (Waltl 1838; Küster 1848; Mulsant and Rey 1863; Ganglbauer 1884; Bodemeyer 1906; Pic 1913, 1914, 1917a, 1917b; 
Breuning 1947, 1962a; Demelt and Alkan 1962; Demelt 1963; Braun 1978a; Althoff and Danilevsky 1997; Pesarini and Sabbadini 2008; Löbl and Smetana 2010; Özdikmen 2010, 2012; Danilevsky 2015a; Özdikmen and Kaya 2015g)

Range: E: BU TR A: TR

*N. orientale Ganglbauer, 1884: 510 (N. balcanicum var.)

Type information: Syntypes $q+$, ex collection L. Ganglbauer, Naturhistorisches Museum Wien [type locality 'Kleinasien' (Turkey)]

flaschneri Pic, 1890: clxxv [Turkey: İstanbul: Beykoz]

dispar Pic, 1892b: xxvii (N. flaschneri var.) [Turkey]

merkli Pic, 1893: cxcvi (N. flaschneri var.) [Turkey]

eduardi Pic, 1900c: 82 (N. flaschneri var.) [Turkey]

Provincial distribution in Turkey: TR-A: IS IZ KO (Ganglbauer 1884; Pic 1890, 1892b, 1893, 1900c; Bodemeyer 1906; Demelt and Alkan 1962; Breuning 1962a; Demelt 1963; Braun 1978a; Pesarini and Sabbadini 2008; Löbl and Smetana 2010; Özdikmen 2010, 2012; Danilevsky 2015a; Özdikmen and Kaya 2015g)

Range: A: TR

N. pelleti Mulsant and Rey, 1863: 149 (Dorcadion)

Type information: Holotype, ex collection Petri Pellet, Muséum d'Histoire Naturelle de Perpignan [type locality 'Smyrne' (Turkey: İzmir)]

segne Mulsant and Rey, 1863: 155 (Dorcadion) [Turkey: İzmir]

disjunctum Pic, 1917b: 6 (N. segne var.) ['?Serbia and Montenegro: Beograd' probably mislabeled]

credulum Pic, 1917a: 10 (N. segne var.) [Turkey]

Provincial distribution in Turkey: TR-A: IS IZ; TR-E: IS (Mulsant and Rey 1863; Ganglbauer 1884; Bodemeyer 1906; Pic 1917a; Villiers 1959; Breuning 1962a; Pesarini and Sabbadini 2008; Löbl and Smetana 2010; Özdikmen 2010, 2012; Danilevsky 2015a; Özdikmen and Kaya 2015g)

Range: E: GR TR A: TR

\section{Dorcadionini species with uncertain status in Turkish fauna}

Some Dorcadionini species that were recorded from Turkey erroneously and whose occurrences in Turkey need confirmation are excluded from the list and included here.

\section{Dorcadion (Carinatodorcadion)}

Dorcadion carinatum (Pallas, 1771) - This species has been recorded by a few authors from NE Anatolia. It has five subspecies from European Russia, Kazakhstan, Ukraine and Azerbaijan. The subspecies structure of this species was revised by Danilevsky (1998). According to Danilevsky (1998) distribution patterns of $D$. carinatum never reach Turkey in the South. Therefore, the presence of this species in Turkey is uncertain, in my opinion.

\section{Dorcadion (Cribridorcadion)}

Dorcadion arenarium (Scopoli, 1763) - This species has been recorded by a few authors from NC and NW Anatolia. It has 11 subspecies from France, Italy, Croatia, 
Bosnia and Herzegovina, Slovenia and Albania. Therefore, the presence of this species is impossible for Turkey.

Dorcadion atticum Kraatz, 1873 - This species has been reported only by Lodos (1998) for Turkey in his unrealistic list without any exact locality. However, the species has been recorded only from Greece now. It is endemic to Greece. Therefore, it is impossible for it to occur in Turkey.

Dorcadion auratum Tournier, 1872 - This species has been reported only by Lodos (1998) for Turkey in his unrealistic list without any exact locality. However, the species has been recorded only from Georgia now. It is endemic to Georgia. Therefore, it is impossible for it to occur in Turkey.

Dorcadion beckeri Kraatz, 1873 - This species has been recorded by a few authors from $\mathrm{N}$ and $\mathrm{E}$ Anatolia. It has two subspecies from South European Russia and Azerbaijan. Therefore, the presence of this species in Turkey is impossible.

Dorcadion cinerarium (Fabricius, 1787) - This species has been recorded by various authors from different parts of Anatolia. It has 19 subspecies from European Russia, Ukraine, Moldavia and Azerbaijan. According to Lazarev (2009), Dorcadion populations from Turkey were wrongly regarded as different forms of $D$. cinerarium ( $D$. micans Thomson, 1867, D. sericatulum Kraatz, 1873 and D. macropus Kraatz, 1873). He also stated that Dorcadion populations that were attributed before to $D$. cinerarium from Turkey (as subspecies, variations, morphs, forms or aberrations) belong to another partly undescribed species. Therefore, the presence of this species in Turkey is doubtful.

Dorcadion decipiens (Germar, 1824) - This species recorded by Önalp (1991) and Lodos (1998) from Turkey without any exact locality. It is known from Hungary, Moldavia, Romania, Ukraine, Yugoslavia and Bulgaria. Önalp (1991) mentioned a male specimen in the Senckenberg Museum and one female specimen in her collection from Turkey. Therefore, the presence of this species in Turkey is uncertain.

Dorcadion elegans Kraatz, 1873 - This species has been reported only by Lodos (1998) for Turkey in his unrealistic list without any exact locality. However, the species has been recorded only from South European Russia, Ukraine and Kazakhstan now. Therefore, it is impossible for it to occur in Turkey.

Dorcadion etruscum (Rossi, 1790) - This species has been reported only by Lodos (1998) for Turkey in his unrealistic list without any exact locality. However, the species has been recorded only from Italy now. It is diffuse in Italy, Greece and Albania. Therefore, it is impossible for it to occur in Turkey.

Dorcadion glaucum Faldermann, 1837 - This species has been reported only by Lodos (1998) for Turkey in his unrealistic list without any exact locality. However, the species has been recorded only from Iran now. Therefore, it is impossible for it to occur in Turkey. 
Dorcadion holosericeum Krynicki, 1832 - This species has been reported only by Lodos (1998) for Turkey in his unrealistic list without any exact locality. It has two subspecies from European Russia, Kazakhstan, Belarus, Moldavia, Poland, Romania, Ukraine and Georgia. Therefore, it is impossible for it to occur in Turkey.

Dorcadion impressicolle Kraatz, 1873 - This species has been reported only by Lodos (1998) for Turkey in his unrealistic list without any exact locality. However, the species has been recorded only from Syria now. Therefore its presence in Turkey is uncertain.

Dorcadion indutum Faldermann, 1837 - This species has been reported only by Lodos (1998) for Turkey in his unrealistic list without any exact locality. However, the species has been recorded only from Armenia and Azerbaijan now. Therefore, it is impossible for it to occur in Turkey.

Dorcadion investitum Breuning in Braun, 1978a: 111 - This species, as attributed to Breuning, has been recorded only by Braun (1978a) from Erzurum province in NE Anatolia. It is a nomen nudum. Therefore, it is removed from the list.

Dorcadion litigiosum Ganglbauer, 1884 - This species has been reported only by Lodos (1998) for Turkey in his unrealistic list without any exact locality. It has two subspecies from Romania, ?Moldavia and Ukraine now. Therefore, it is impossible for it to occur in Turkey.

Dorcadion mediterraneum Breuning, 1942 - This species has been reported only by Lodos (1998) for Turkey in his unrealistic list without any exact locality. However, the species has been recorded only from Italy now. It is endemic to Italy. Therefore, it is impossible for it to occur in Turkey.

Dorcadion minutum Kraatz, 1873 - This species has been reported only by Lodos (1998) for Turkey in his unrealistic list without any exact locality. It has two subspecies from Greece. It is endemic to Greece. Therefore, it is impossible for it to occur in Turkey.

Dorcadion murrayi Küster, 1847 - This species has been reported only by Lodos (1998) for Turkey in his unrealistic list without any exact locality. However, the species has been recorded only from Romania and Yugoslavia now. Therefore, it is impossible for it to occur in Turkey.

Dorcadion pusillum Küster, 1847 - This species recorded only by Lodos (1998) from Turkey in his unrealistic list without any exact locality. It has three subspecies from Moldavia, Romania, Ukraine and South European Russia now. Therefore, the presence of this species in Turkey is impossible.

Dorcadion reitteri Ganglbauer, 1884 - This species has been reported only by Lodos (1998) for Turkey in his unrealistic list without any exact locality. However, the species has been recorded only from Georgia now. It is endemic to Georgia. Therefore, it is impossible for Turkey. 
Dorcadion rosinae K. Daniel in Bodemeyer, 1900: 127 - This species, as attributed to K. Daniel, has been recorded only by Bodemeyer (1900) from Konya province (Akşehir, Sultan Mt.) in C Anatolia. It is a nomen nudum. Therefore, it is removed from the list.

Dorcadion sareptanum Kraatz, 1873 - This species has been reported only by Lodos (1998) for Turkey in his unrealistic list without any exact locality. It has four subspecies from South European Russia, Kazakhstan and Ukraine now. Therefore, it is impossible for it to occur in Turkey.

Dorcadion scopolii (Herbst, 1784) - This species has been reported only by Lodos (1998) for Turkey in his unrealistic list without any exact locality. However, the species has been recorded from Bulgaria, Croatia, Czech Republic, Hungary, Moldavia, Poland, Romania, Slovakia, Ukraine and Yugoslavia now. Therefore, the presence of this species in Turkey is uncertain.

Dorcadion semilucens Kraatz, 1873) - This species has been recorded by a few authors from Anatolia. However, the species is known only from Armenia and Azerbaijan now. Therefore, the presence of this species in Turkey is uncertain.

Dorcadion seminudum Kraatz, 1873 - This species has been recorded only by Plavilstshikov (1958) and Lodos (1998) from NE Anatolia. However, the species is known only from Armenia and Azerbaijan now. Therefore, the presence of this species in Turkey is uncertain.

Dorcadion sericatum Sahlberg, 1823 - This species has been recorded by various authors from different parts of Anatolia. It is endemic to Crimea now. Therefore, the presence of this species in Turkey is impossible.

Dorcadion tuerki Ganglbauer, 1884 - This species has been recorded only by Demelt (1963) from Amanos Mountains in South Anatolia. It is endemic to Iran now. Therefore, the presence of this species in Turkey is impossible.

Neodorcadion (s. str.)

Neodorcadion fallax (Kraatz, 1873) - This species has been reported only by Lodos (1998) for Turkey in his unrealistic list without any exact locality. However, the species is recorded only from Bulgaria, Greece and Macedonia now. Therefore, the presence of this species in Turkey is uncertain.

\section{Results and discussion}

This work provides essential information to understand and conserve the existing biodiversity of Turkey (a particular region). Although the Dorcadionini of Turkey is not well studied, there is always a need to present updated information because of changes in the taxonomic nomenclature and many surveys resulting in new descriptions, records and localities. This is a crucial attempt to present a synthesised and updated list of Dorcadionini of Turkey. In all, 278 species-group taxa [Dorcadion: 265 species-group taxa; Megalodorcadion: seven species; 
and Neodorcadion: six species) are listed. Eodorcadion, Iberodorcadion and Politodorcadion have no species in Turkey. The Dorcadionini fauna of Turkey presents a remarkable endemism with 227 species group taxa, which constitute about $82 \%$ of the total number. This is surely a result of hotspots included in the area. We suggest future investigations in localities that are not well collected. Forthcoming surveys will most likely contribute to the Dorcadionini fauna of Turkey with new records, and new species and subspecies.

\section{Disclosure statement}

No potential conflict of interest was reported by the author.

\section{References}

Adlbauer K. 1982. Eine neue Dorcadion-Art aus Kleinasien (Coleoptera, Cerambycidae). Nachrichtenblatt der Bayerischen Entomologen. 31:104-107.

Adlbauer K. 1988. Neues zur Taxonomie und Faunistik der Bockkäferfauna der Türkei (Coleoptera, Cerambycidae). Entomofauna. 9:257-297.

Adlbauer K. 1992. Zur Faunistik und Taxonomie der Bockkäferfauna der Türkei II (Coleoptera, Cerambycidae). Entomofauna. 13:485-509.

Al-Hamadani NDS, Özdikmen H. 2014. Longicorn beetles of Çankırı province in Turkey (Coleoptera: Cerambycidae). Munis Entomol Zool. 9:931-941.

Althoff J, Danilevsky ML. 1997. A check-list of longicorn beetles (Coleoptera, Cerambycoidea) of Europe. Ljubljana: Slovensko Entomološko Društvo Štefana Michielija; p. 64.

Anichtchenko AV, Shavrin AV. 2013. The type specimens of Coleoptera (Insecta) deposited in the Beetle collection (DUBC) of Daugavpils University, Latvia. Acta Biologica Universitatis Daugavpiliensis. 13:1-10.

Bernhauer D. 1988. Türkische Dorcadien (1. Beitrag) (Coleoptera: Cerambycidae: Lamiinae). Entomologische Zeitschrift (Essen). 98:97-112.

Bernhauer D. 2010. Eine neue Art der Gattung Dorcadion Dalm. aus der Türkei, nördlich von Anamur (Coleoptera, Cerambycidae, Lamiinae). 2. Beitrag zur Kenntnis der türkischen Dorcadion. Coleoptera, Schwanfelder Coleopterologische Mitteilungen. 14:189-194.

Bernhauer D, Peks H. 2010. Zwei neue Arten der Gattung Dorcadion Dalm. aus der Türkei, nördlich von Anamur (Coleoptera, Cerambycidae, Lamiinae), sowie eine neue Unterart zu Dorcadion lodosi Sabbadini und Pesarini. 3. Beitrag zur Kenntnis der türkischen Dorcadion. Coleoptera, Schwanfelder Coleopterologische Mitteilungen. 14:195-206.

Bernhauer D, Peks H. 2011a. Fünf neue Arten der Gattung Dorcadion Dalm. Aus dem mittleren und östlichen Taurus. (Coleoptera, Cerambycidae, Lamiinae). 6. Beitrag zur Kenntnis der türkischen Dorcadion. Coleoptera, Schwanfelder Coleopterologische Mitteilungen. 15:215-227.

Bernhauer D, Peks H. 2011b. Vier neue Arten der Gattung Dorcadion Dalm. aus der Nord-WestTürkei. (Coleoptera, Cerambycidae, Lamiinae). 5. Beitrag zur Kenntnis der türkischen Dorcadion. Coleoptera, Schwanfelder Coleopterologische Mitteilungen. 15:201-214.

Bernhauer D, Peks H. 2012. Vier neue Arten der Gattung Dorcadion Dalm. aus der Osttürkei. (Coleoptera, Cerambycidae, Lamiinae). 7. Beitrag zur Kenntnis der türkischen Dorcadion. Entomologische Blätter und Coleoptera. 108:209-221.

Bernhauer D, Peks H. 2013a. Drei neue Arten der Gattung Dorcadion Dalman, 1817 aus der SüdostTürkei, sowie drei neue Unterarten von D. (Cribridorcadion) goelbasiense sp.n. (Coleoptera, Cerambycidae, Lamiinae). 8. Beitrag zur Kenntnis der türkischen Dorcadion. Entomologische Blätter und Coleoptera. 109:309-323.

Bernhauer D, Peks H. 2013b. Vier neue Arten der Gattung Dorcadion Dalman, 1817 aus der Nordtürkei, (Coleoptera, Cerambycidae, Lamiinae). 9. Beitrag zur Kenntnis der türkischen Dorcadion. Entomologische Blätter und Coleoptera. 109:325-336. 
Bernhauer D, Peks H. 2014a. Fünf neue Arten der Gattung Dorcadion Dalman, 1817 aus der Zentral- und Osttürkey (Coleoptera, Cerambycidae, Lamiinae). 10. Beitrag zur Kenntnis der türkischen Dorcadion. Entomologische Blätter und Coleoptera. 110:101-118.

Bernhauer D, Peks H. 2014b. Dorcadion skoupyorum nom. nov. for Dorcadion skoupyi Bernhauer \& Peks, 2014 (Coleoptera, Cerambycidae). Human Space Int Almanac. 3:191-192.

Bilberg GJ. 1817. [New taxa]. In: Schönherr CJ, editor. Appendix ad C. J. Schönherr Synonymiam Insectorum. Descriptiones Novarum Specierum Insectorum. Scaris, Lewerentziana. Stockholm. 1:1-266.

Bodemeyer HEV. 1900. Quer durch Klein Asien, in den Bulghar Dagh; Eine Naturvissenschafliche studien-Reise. Emmendingen: Coleopterologisches; p. 196.

Bodemeyer HEV. 1906. Beitrage zur Käferfauna von Klein Asien. Deutsche Entomologische Zeitschrift. 2:417-437.

Braun W. 1975. Beitrag zur Kenntnis der Gattung Dorcadion (Col., Cerambycidae, Lamiinae). Entomologische Zeitschrift. 85:17-21.

Braun W. 1976a. 3. Beitrag zur Kenntnis der Dorcadien Anatoliens. Dorcadion ladikanum n. nom. Und Bemerkungen zur Kenntnis des Dorcadion ardahense Breuning (Col., Cerambycidae). Entomologische Zeitschrift (Stuttgart). 86:173-175.

Braun W. 1976b. 4. Beitrag zur Kenntnis der Dorcadien Anatoliens. Neue Dorcadien-Arten und Bemerkungen zur Variationsbreite des D. mesopotamicum Breun. (Col., Cerambycidae). Entomologische Zeitschrift (Stuttgart). 86:254-264.

Braun W. 1976c. 2. Beitrag zur Kenntnis der Dorcadien Anatoliens (Col., Cerambycidae). Entomologische Zeitschrift (Stuttgart). 86:54-60.

Braun W. 1978a. Die Dorcadienausbeute der Forschungsreisen von W. Heinz 1963-1977, Faunistiche Aufstellung, Beschreibung einer neuen Unterart und Bemerkungen zur Systematik wenig bekannter Arten (Coleoptera, Cerambycidae). Nachrichtenblatt der Bayerischen Entomologen. 27:101-116.

Braun W. 1978b. Ein neue Art der Gattung Dorcadion aus Anatolien (Col.: Cerambycidae). Entomologische Zeitschrift (Stuttgart). 88:185-187.

Braun W. 1979. Beitrag zur Kenntnis der Gattung Dorcadion Systematisch neu bewertete Dorcadion-Formen (Col., Cerambycidae). Nachrichtenblatt der Bayerischen Entomologen. 28:81-86.

Breit J. 1923. Beitrag zur Kenntnis der Dorcadion-Arten des Balkans. Wiener Entomologische Zeitung. 40:145-149.

Breuning S. 1942. Novae species Cerambycidarum. XI. Folia Zoologica et Hydrobiologica. 11:113175.

Breuning S. 1943. Nouveaux Cérambycides paléarctiques (1 re note). Miscellanea Entomologica. 40:89-104.

Breuning S. 1944. Nouveaux Cerambycides paléarctiques (2e note). Miscellanea Entomologica. 41:11-16.

Breuning S. 1946. Nouvelles formes de Dorcadion (Col. Cerambycidæ). Miscellanea Entomologica. 43:91-132.

Breuning S. 1947. Nouvelles formes de Dorcadion (Col., Cerambycidæ) 2e note. Miscellanea Entomologica. 43:149-172.

Breuning S. 1948. Nouvelles formes de Dorcadion (Col., Cerambycidae). (3e note). Miscellanea Entomologica. 45:57-64.

Breuning S. 1955. Beschreibung einer neuen Rasse des Dorcadion divisum Germ. (Ceramb., Coleoptera). Annales Historico-Naturales Musei Nationalis Hungarici, Budapest (Ser. Nov.). 6:263.

Breuning S. 1956. Deux nouveaux Lamiaires de Sumatra et deux nouvelles formes de Dorcadion du Musée de Budapest (Coleoptera, Cerambycidae). Annales Historico-Naturales Musei Nationalis Hungarici (series nova). 7:403-404.

Breuning S. 1960. Nouvelles formes de Lamiaires. (douzième partie). Bulletin de l'Institut Royal des Sciences Naturelles de Belgique (Bruxelles). 36:1-30. 
Breuning S. 1962a. Revision der Dorcadionini. Entomologische abhandlungen und berichte aus dem staatliche museum für tierkunde in Dresden. 27:665.

Breuning S. 1962b. Neue und interessante Formen der Gattungen Carabus L. und Dorcadion Muls. aus Anatolien. Reichenbachia (Dresden). 1:37-40.

Breuning S. 1963. Weiterer Beitrag zur Kenntnis der Gattung Dorcadion Dalm. (Col. Ceramb.). Zeitschrift der Arbeitsgemeinschaft Österreichischer Entomologen. 15:35-36.

Breuning S. 1964a. Nouvelles formes de Lamiaires. (Seizième partie). Bulletin de l'Institut Royal des Sciences Naturelles de Belgique (Bruxelles). 40:1-8.

Breuning S. 1964b. Quatre nouvelles espèces du genre Dorcadion Dalm. (Coleoptera Cerambycidae). Bolletino dell'Associazione Romana di Entomologia. 19:31-32.

Breuning S. 1966a. Nouvelles formes de Lamiaires (dix-septième partie). Bulletin de l'Institut Royal des Sciences Naturelles de Belgique (Bruxelles). 42:1-22.

Breuning S. 1966b. Deux nouvelles espèces du genre Dorcadion Dalm. d'Anatolie (Coleoptera, Cerambycidae). Bollettino della Società Entomologica Italiana (Genova). 96:145-147.

Breuning S. 1969. Quelques formes nouvelles du genre Dorcadion Dalm. de Grèce (Coleoptera Cerambycidae). Bolletino dell'Associazione Romana di Entomologia. 24:42.

Breuning S. 1970. Nouveaux Dorcadion des collections du Muséum de Paris. L'Entomologiste (Paris). 26:97-101.

Breuning S. 1973. Beschreibung zweier neuer Arten der Gattung Dorcadion (Col., Cerambycidae, Lamiinae). Entomologische Zeitschrift (Frankfurt an Main). 83:54-55.

Breuning S. 1974a. Beschreibung neuer Arten der Gattung Dorcadion Dalm. (Coleoptera: Cerambycidae). Mitteilungen der Entomologischen Gesellschaft (Basel, NF). 24:148-154.

Breuning S. 1974b. Descriptions de Dorcadions nouveaux d'Anatolie (Coleoptera, Lamiinae). Bulletin de la Société Entomologique de Mulhouse [octobre-novembre-décembre]. 1974:65.

Breuning S. 1975. Descriptions de Dorcadions nouveaux d'Anatolie (Coleoptera Lamiinae). Bulletin de la Société Entomologique de Mulhouse. 1975:10.

Breuning S. 1977. Quelques rectifications systématiques sur les Lamiaires (Col. Cérambycidae). Bulletin de la Société Entomologique de Mulhouse [Octobre-Décembre]. 1977:59-60.

Breuning S. 1979. Beschreibung einer neuen species der Gattung Dorcadion (Col.: Cerambycidae). Entomologische Zeitschrift (Stuttgart). 89:92-93.

Breuning S. 1981. Descriptions de quelques nouvelles espèces de Lamiinae (Col., Cerambycidae) de la coll. Bremer. Entomologische Blätter für Biologie und Systematik der Käfer. 77:181-182.

Breuning S, Ruspoli M. 1971. Description de deux espèces nouvelles de Cerambycidae (Col.) d'Anatolie. Entomops (Nice). 20:127-128.

Breuning S, Ruspoli M. 1975. Description d'une espèce nouvelle de Cerambycidae (Col.) d'Anatolie. Entomops (Nice). 36:116.

Breuning S, Villiers A. 1967. Cérambycides de Turquie (2. note). L'Entomologiste. 23:59-63.

Chevrolat LAA. 1882. Espèces nouvelles de Longicornes européens et circa-méditerranéens et Remarques diverses. Annales de la Société Entomologique de France, Paris. 2:57-64.

Chevrolat LAA. 1856. Coléoptères nouveaux. Revue et Magasin de Zoologie (Paris). 8:84-89.

Cihan N, Özdikmen H, Aytar F. 2013. Contributions of the longhorned beetles knowledge of Turkey by the subfamilies Prioninae, Lepturinae, Dorcadioninae and Lamiinae (Coleoptera: Cerambycidae). Munis Entomol Zool. 8:883-894.

Çıplak B. 2003. Distribution of Tettigoniinae (Orthoptera, Tettigoniidae) bush-crickets in Turkey: the importance of the Anatolian Taurus Mountains in biodiversity and implications for conservation. Biodiv Conserv. 12:47-64.

Çıplak B. 2004. Biogeography of Anatolia: the marker group Orthoptera. Mem Soc Entomol Ital. 82:357-372.

Conservation International: Biodiversity Hotspots [Internet].c. 2015. Arlington. Virginia: Center for Applied Biodiversity Science at Conservation International; [cited 2015 Aug 15]. Available from: http: //www.biodiversityhotspots.org/

Dalman JW. 1817. Descriptiones novarum specierum insectorum. In: Schönherr CJ, editor. Synonymia insectorum, oder: Versuch einer Synonymie aller bisher bekannten Insecten; nach 
Fabricii Systema Eleutheratorum \& c. geordnet. Eleutherata oder Käfer. Lewerentzischen Buch., Skara. 1: 506 pp.

Daniel K. 1900. Vorläufige Diagnosen. Societas Entomologica. 15:139-140.

Daniel K, Daniel J. 1903. Nova, von Hauptmann v. Bodemeyer in Kleinasien gesammelt. 2. Serie. Münchener Koleopterologische Zeitschrift. 1:319-333.

Danilevsky ML. 1993. New species of Cerambycidae (Coleoptera) from East Asia with some new records. Annales Historica-Naturales Musei Nationalis Hungarici. 84:111-116.

Danilevsky ML. 1996. New taxa of the genus Dorcadion Dalman from Asia (Coleoptera, Cerambycidae). Lambillionea. 96:407-420.

Danilevsky ML. 1998. A revue of subspecific configuration of Dorcadion (Carinatodorcadion) carinatum (Pallas, 1771) with a description of new subspecies (Coleoptera: Cerambycidae). Acta Entomologica Slovenica. 6:135-142.

Danilevsky ML. 1999. Two new subspecies of Dorcadion scabricolle Dalman, 1817 close to $D$. scabricolle sevangense Reitter, 1889 from Transcaucasia (Coleoptera, Cerambycidae). Lambillionea. 99:25-29.

Danilevsky ML. 2009. Species group taxa of longhorned beetles (Coleoptera, Cerambycidae) described by N. N. Plavilstshikov and their types preserved in the zoological museum of the Moscow State University and in the Zoological Institute of the Russian Academy of Sciences, St. Petersburg. Entomol Rev (Washington). 89:689-720.

Danilevsky ML. 2012. Additions and corrections to the new catalogue of palaearctic Cerambycidae (Coleoptera) edited by I. Löbl and A. Smetana, 2010. Part. III. Munis Entomol Zool. 7:109-173.

Danilevsky ML. 2014. Two new subspecies of Dorcadion aethiops (Scopoli, 1763) from Bulgaria (Coleoptera, Cerambycidae). Human Space Int Almanac. 3:251-254.

Danilevsky ML. 2015a. Catalogue of Palaearctic Cerambycoidea [cited 2015 Mar 8]. Available from: http://www.cerambycidae.net/catalog.pdf

Danilevsky ML. 2015b. A new species of genus Dorcadion Dalman, 1817 (Coleoptera, Cerambycidae) from Turkey (Ispir, Erzurum). Human Space Int Almanac. 4:220-223.

Danilevsky ML, Kasatkin DG. 2007. Further investigation of Dorcadionini (Coleoptera: Cerambycidae) endophallus with a revison of taxonomical position of the genus Trichodorcadion Breuning, 1942. Russ Entomol J. 15:401-407.

Danilevsky ML, Kasatkin DG, Rubenyan AA. 2005. Revision of the taxonomic structure of the tribe Dorcadionini (Coleoptera, Cerambycidae) on the base of endophallic morphology. Russ Entomol J. 13:127-149.

Danilevsky ML, Murzin SV. 2009. A revue of Dorcadion Dalman, 1817 species of "laeve-group" Part I (Coleoptera, Cerambycidae, Lamiinae). Les Cahiers Magellanes. 92:1-20.

Dascalu M-M, Fusu L. 2012. Dorcadion axillare Küster, 1847 (Coleoptera, Cerambycidae): distribution, morphometrics, karyotype and description of a new subspecies from Romania. Zootaxa. 3322:35-48.

Demelt CV. 1963. Beitrag zur Kenntnis der Cerambycidenfauna Kleinasiens und 13. Beitrag zur Biologie palaearkt. Cerambyciden, sowie Beschreibung einer neuen Oberea-Art. Entomologische Blatter. 59:132-151.

Demelt CV. 1967. Nachtrag zur Kenntnis der Cerambyciden-Fauna Kleinasiens. Entomologische Blatter. 63:106-109.

Demelt CV, Alkan B. 1962. Short information of Cerambycidae Fauna of Turkey. Bitki Koruma Bülteni. 2:49-56.

Desbrochers des Loges J. 1870. Descriptions de Coléoptères nouveaux d'Europe et confins. L'Abeille. 7:97-135.

Ekiz AN, Şen I, Aslan EG, Gök A. 2013. Checklist of leaf beetles (Coleoptera: Chrysomelidae) of Turkey, excluding Bruchinae. J Nat Hist. 47:2213-2287.

Fairmaire LMH. 1866. Notice sur les Coléoptères récoltés par M. J. Lédérer sur le Bosz-Dagh (Asie Mineure). Annales de la Société Entomologique de France (Paris). 6:249-280.

Faldermann F. 1837. Fauna entomologica Transcaucasica. Coleoptera 2. Longicornes vel Capricornes. Nouveaux Mémoires de la Société Impériale des Naturalistes de Moscou. 5:1-433. 
Ferrari GA. 1864. Zwei neue Dorcadion-Arten vom Caucasus beschrieben vom süddeutschen Entomologen. Wiener entomologische Monatschrift. 8:479-483.

Fischer von Waldheim G. 1823a. Entomographie de la Russie, et genres des Insectes. Entomographia Imperii Rossici, sue Caesareae Majestati Alexandro I dicata. 2: plates 18-50.

Fischer von Waldheim G. 1823b. Entomographie de la Russie, et genres des Insectes. Entomographia Imperii Rossici, sue Caesareae Majestati Alexandro I dicata. 2: 264 pp.

Fairmaire LMH. 1884. Liste des Coléoptères recueillis par M. I'abbé David à Akbès (Asie-Mineure) et Descriptions des Espèces Nouvelles. Annales de la Société Entomologique de France. 4:165-180.

Frivaldszky von Frivald I. 1835. Á Magyar Tudós Társaság' Évkönyvei. Második Kötet. Á Magyar Tudós Társaság' Évkönyvei. 2:251-276.

Frivaldszky von Frivald I. 1837. Ujabb Közlésci az általa eszközlött, balkányi természettudományi utazásról. Á Magyar Tudós Társaság' Évkönyvei. 3:156-184.

Frivaldszky von Frivald I. 1845. A Királyi Magyar természettudományi társulat évkönyvei I. Annalen der Ungarischen Naturforschenden Gesellschaft, Pesten. 1:161-187.

Fuchs E, Breuning S. 1971. Die Cerambycidenausbeute der Anatolienexpedition 1966-67 des Naturhistorischen Museums, Wien. Annalen Naturhistorischen Museum Wien. 75:435-439.

Ganglbauer L. 1882. Über Dorcadion graecum Waltl. Wiener Entomologische Zeitung. 1:228.

Ganglbauer L. 1884. Bestimmungs-Tabellen der europaeischen Coleopteren. VIII. Cerambycidae. Verhandlungen der Kaiserlich-Königlichen Zoologish-Botanischen Gesellschaft in Wien. 33:437-586.

Ganglbauer L. 1886. Neue und weniger bekannte Longicornier des paläarktischen Faunengebietes. Verhandlungen der Kaiserlich-Königlichen Zoologisch-Botanischen Gesellschaft in Wien. 35:515-524.

Ganglbauer L. 1897. Cerambycidae. Bestimmt und beschrieben von L. Ganglbauer. In K. Escherich: zoologische Ergebnisse einer von Dr. K. Escherich und Dr. L. Kathariner nach Central-Kleinasien unternommenen Reise. IV. Theil. Coleopteren. Stettiner Entomologische Zeitung. 58:1-69.

Germar EF. 1824. Insectorum species novae aut minus cognitae, descriptionibus illustratae. Coleopterorum Species. Hendelii et filii, Halae Volumen primum. Coleoptera: xxiv + 1-624.

Germar EF. 1839. Fauna Insectorum Europae. Fasc. 21. Halae: C. A. Kümmelii; p. 1-25.

Gfeller W. 1972. Cerambycidae (Coleoptera) der Türkei-Persienexpedition 1970 der Herren Dr. H. c. W. Wittmer und U. v. Botmer. Mitteilungen der Entomologischen Geselschaft Basel. 22:1-8.

Gistel JNFX. 1848. Naturgeschichte des Thierreichs für höhere Schulen. Hoffmann, Stuttgart. 216 pp.

Gül-Zümreoğlu S. 1972. Catalogue of Insect and common pests (1928-1969). Bornova: T. C. Publications of Agriculture Ministry; p. 119.

Gül-Zümreoğlu S 1975. Investigations on taxonomy, host plants and distribution of the longhorned beetles (Cerambycidae-Coleoptera) in Aegean Region. T. C. Ministry of Food, Agriculture and Stockbreeding, No: 28. İzmir: İstiklal Press; 208 pp.

Hampe C. 1852. Verzeichniß der von mir im Kaukasus, in Trans-Kaukasien, Armenien, Kurdistan und West-Persien gesammelten und von Dr. Hampe bestimmten Koleopteren. In Moritz Wagner, editor. Reise nach Persien und dem Lande der Kurden. 2:302-315.

Herbst JFW. 1784. Kritisches Verzeichniß meiner Insektensammlung. Archiv der Insectengeschichte. 5:1-151.

Heyden L. 1884. Beitrag zur Coleopterenfauna der Insel Askold und anderer Theile des Amurgebietes. Deutsche entomologische Zeitschrift. 28:273-300.

Heyden L. 1888. Neue und interessante Coleopteren aus Malatia in Mesopotamien. Deutsche Entomologische Zeitschrift. 32:72-78.

Heyden L. 1894. Ueber Meloe Olivieri Chevrolat und sechs neue Longicornen aus Klein-Asien. Deutche Entomologische Zeitschrift. 1894:85-90.

Heyrovský L. 1932. Weitere neue palaearktische Cerambyciden-formen. Entomologisches Nachrichtenblatt (Troppau). 6:103-104.

Heyrovský L. 1943. Príspevek k faune tribu Dorcadiini Thrácie (Col., Ceramb.). Casopis Ceské Spolecnosti Entomologické. 40:77-80.

Heyrovský L. 1962. Zwei neue Dorcadion-Arten aus der Balkanhalbinsel. Bulgarische Akademia der Wissenschaften. Mitteilungen des zoologischen Institutes und Museums. 12:225-226. 
Heyrovský L. 1964. Eine neue Dorcadion-Art aus Kleinasien (Coleoptera, Cerambycidae). Reichenbachia (Dresden). 3:97-98.

Holzschuh C. 1977. Neue Bockkäfer aus Anatolien und Iran (Col., Cerambycidae). Koleopterologische Rundschau (Wien). 53:127-136.

Holzschuh C. 1980. Revision einer Cerambycidenausbeute des Naturhistorischen Museums Wien (Coleoptera). Annalen des Naturhistorischen Museums in Wien. 83:573-574.

Holzschuh C. 1982. Elf neue Bockkäfer aus Europa und Asien (Col.: Cerambycidae). Zeitschrift der Arbeitsgemeinschaft Österreichischer Entomologen. 33:65-76.

Holzschuh C. 1989. Beschreibung neuer Bockkäfer aus Europa und Asien (Cerambycidae, Col.). Koleopterologische Rundschau (Wien). 59:153-183.

Holzschuh C. 1991. 63 neue Bockkäfer aus Asien, vorwiegend aus China und Thailand (Coleoptera: Disteniidae und Cerambycidae). Schriftenreihe der Forstlichen Bundesversuchanstalt (FbvaBerichte) (Wien). 60:5-71.

Holzschuh C. 1995. Beschreibung von 65 neuen Bockkäfern aus Europa und Asien, vorwiegend aus Thailand und China (Coleoptera: Disteniidae und Cerambycidae). Schriftenreihe der Forstlichen Bundesversuchanstalt (Fbva-Berichte) (Wien). 84:1-63.

Holzschuh C. 2007. Beschreibung von 80 neuen Bockkäfern aus der orientalischen und palaearktischen Region, vorwiegend aus China, Laos und Borneo (Coleoptera, Cerambycidae). Entomologica Basiliensa et Collectionis Frey. 29:177-286.

Jakovlev Al. 1901. Deux nouvelles espèces de Dorcadion Dalm. De I'Asie mineure (Coleoptera, Cerambycidae). Revue Russe d'Entomologie (Saint-Petersbourg). 1:83-87.

Jakovlev BE. 1899. Nouvelles espèces du genre Dorcadion Dalm. Horae Soc Entomol Ross (St. Petersbourg). 34:59-70.

Jurecek Š. 1931. Dve nové formy tesariku z Recka a Malé Azie. Casopis Ceské Spolecnosti Entomologické. 28:124.

Jureček Š. 1933: Noví tesaříci palearktické fauny. Casopis Ceské Spolecnosti Entomologické. 30:127-130.

Kadlec S. 2006. Two new species of the genus Dorcadion Dalman, 1817 from Iran and Turkey (Col.: Cerambycidae: Lamiinae). Animma.x. 12:8-15.

König EG. 1901. Erster Beitrag zur Coleopteren-Fauna des Kaukasus. Wiener Entomologische Zeitung. 20:9-10.

Konstantinov AS, Korotyaev BA, Volkovitsh MG. 2009. Insect biodiversity in the Palearctic region. Chapter 7. In: Foottit RG, Adler PH, editors. Insect biodiversity: science and society. Oxford: Wiley-Blackwell; p. 107-162.

Kraatz G. 1871. Dorcadion formosum n. sp. Berliner Entomologische Zeitschrift. 14:411-412.

Kraatz G. 1873. Die Käfer Europas. Nach der Natur beschrieben von Dr. G. Kraatz im Anschluss an die Käfer Europa's von Dr. H. C. Küster. In Küster, 1873. Die Käfer Europas nach der Natur beschrieben. 29:1-101.

Kraatz G. 1884. Neu Käfer-Arten aus Malatia im südlichen Kleinasien. Deutsche Entomologische Zeitschrift (Berlin). 28:231-234.

Kraatz G. 1892. Die Varietäten des Dorcadion equestre Laxman. Deutsche entomologische Zeitschrift. 36:173-174.

Krätschmer OE. 1985. Eine neuer Maculatodorcadion. Beitrag zur Kenntnis der Dorcadion-Fauna Anatoliens (Coleoptera: Cerambycidae: Lamiinae). Entomologische Zeitschrift. 95:24-27.

Krätschmer OE. 1987. Beitrag zur Kenntnis der Dorcadion-Fauna Griechenlands und der Türkei (Coleoptera: Cerambycidae: Lamiinae). Entomologische Zeitschrift (Essen). 97:337-340.

Krynicki J. 1832. Enumeratio Coleopterorum Rossiae meridionalis et praecipue in Universitatis Caesareae Charkoviensis circulo obvenientum, quae annorum 1827-1831 spatio observavit. Bulletin de la Société Impériale des Naturalistes de Moscou. 5:65-179.

Küçükkaykı EC, Şirin Ü, Çalışkan H, Şenyüz Y. 2013. Preliminary work on longhorned beetles fauna of Kaz Dağları (Ida Mountain) and near with two new subspecies (Coleoptera: Cerambycidae). Munis Entomol Zool. 8:50-62.

Küster HC. 1846a. Die Käfer Europa's. Nach der Natur beschrieben. Mit Beiträgen mehrerer Entomologen. Nürnberg, Bauer \& Raspe. 5:1-100. 
Küster HC. 1846b. Die Käfer Europa's. Nach der Natur beschrieben. Mit Beiträgen mehrerer Entomologen. Nürnberg, Bauer \& Raspe. 6:1-100.

Küster HC. 1847. Die Käfer Europa's. Nach der Natur beschrieben. Mit Beiträgen mehrerer Entomologen. Nürnberg, Bauer \& Raspe. 8:1-100.

Küster HC. 1848. Die Käfer Europa's. Nach der Natur beschrieben. Mit Beiträgen mehrerer Entomologen. Nürnberg, Bauer \& Raspe (Julius Merz). 15:1-100.

Küster HC. 1852. Die Käfer Europa's. Nach der Natur beschrieben. Mit Beiträgen mehrerer Entomologen. Nürnberg, Bauer \& Raspe. 25:1-100.

Laxmann E. 1770. Novae Insectorum species. Novi commentarii Academiae Scientiarum Imperialis Petropolitanae. 14:593-604.

Lazarev MA. 2009. Armenian Dorcadion (Coleoptera: Cerambycidae) of "cinerarium-group". Stud Rep District Mus Prague-East Taxon Ser. 5:197-219.

Lazarev MA. 2011. Species group taxa of longhorned beetles (Coleoptera, Cerambycidae) described by B. E. Jakovlev and their types preserved in the Zoological Institute of the Russian Academy of Sciences, St. Petersburg, and in the Siberian Zoological Museum, Novosibirsk. Entomol Rev (Washington). 91:634-644.

Lazarev MA. 2013. New subspecies of Dorcadion scabricolle (Dalman, 1817) of Iran and Azerbaijan (Coleoptera, Cerambycidae). Human Space Int Almanac. 2:222-234.

Lazarev MA. 2014. New subspecies of Dorcadion (Cribridorcadion) mniszechi Kraatz, 1873 from Georgia, Armenia and Turkey (Coleoptera, Cerambycidae). Human Space Int Almanac. 3:698709.

Lingafelter SW, Nearns EH, Tavakilian GL, Monné MÁ, Biondi M. 2014. Longhorned woodboring beetles (Coleoptera: Cerambycidae and Disteniidae) primary types of the Smithsonian Institution. Washington (DC): Smithsonian Institution Scholarly Press; p. v-xviii + 1-390.

Löbl I, Smetana A. 2010. Catalogue of palaearctic coleoptera. Volume 6. Chrysomeloidea. Löbl I, Smetana A, editors. Stenstrup: Apollo Books; p. 1-924.

Lodos N. 1998. Entomology of Turkey VI (general, aplied and faunistic). Ege Ü. Ziraat Fak. Yayınları No: 529. İzmir: E. Ü. Faculty of Agriculture Press; p. 300.

Ménétriés E. 1832. Catalogue raisonné des objets de Zoologie recueillis dans un voyage au Caucase et jusqu'aux frontières actuelles de la Perse entrepris par ordre de S. M. L' Empereur. Saint-Pétersbourg. 4:1-271.

Ménétriés E. 1836. Insectes nouveaux de la Turquie. Bulletin Scientifique de I'Académie Impériale des Sciences de Saint-Petersbourg. 1:149-151.

Motschulsky VI. 1838. Coléoptères du Caucase et des Provinces Transcaucasiennes, recueillis et décrits par T. Victor. Bulletin de la Société Impériale des Naturalistes de Moscou. 11:175-188.

Mulsant É, Rey C. 1863. Longicornes nouveaux ou peu connus. Annales de la Société Linnéenne de Lyon (Nouvelle Série). 10:144-184.

Myers N, Mittermeier RA, Mittermeier CG, Da Fonseca GAB, Kent J. 2000. Biodiversity hotspots for conservation priorities. Nature. 403:853-858.

Okutaner AY, Özdikmen H, Yüksel E, Koçak Y. 2011. Some cytogenetic observations of two Dorcadion Dalman, 1817 species (Coleoptera: Cerambycidae: Lamiinae: Dorcadiini). Munis Entomol Zool. 6:866-876.

Önalp B. 1988. Eine neue Dorcadion-Art aus der Türkei (Coleoptera: Cerambycidae: Lamiinae). Entomologische Zeitschrift (Stuttgart). 98:361-363.

Önalp B. 1990. Systematic researches on Dorcadion Dalman, 1817 species in Turkey (Coleoptera, Cerambycidae: Lamiinae) I. HÜ Eğitim Fakültesi Dergisi. 5:57-102.

Önalp B. 1991. Systematic researches on Dorcadion Dalman, 1817 species in Turkey (Coleoptera, Cerambycidae: Lamiinae) II. HÜ Eğitim Fakültesi Dergisi. 6:191-227.

Özbek H. 1978. Hylotrupes bajulus (L.) Serville in Erzurum and the near, and some others longhorn beetles. Atatürk Üniversitesi Ziraat Fakultesi Dergisi. 9:31-44.

Özdikmen H. 2006. Contribution to the knowledge of Turkish longicorn beetles fauna (Coleoptera: Cerambycidae). Munis Entomol Zool. 1:71-90.

Özdikmen H. 2007. The longicorn beetles of Turkey (Coleoptera: Cerambycidae) part I - black sea region. Munis Entomol Zool. 2:179-422. 
Özdikmen H. 2010. The Turkish Dorcadiini with zoogeographical remarks (Coleoptera: Cerambycidae: Lamiinae). Munis Entomol Zool. 5:380-498.

Özdikmen H. 2011. Longhorned beetles of Bolu province in Turkey (Coleoptera: Cerambycidae). Munis Entomol Zool. 6:210-240.

Özdikmen H. 2012. The longhorned beetles that originally described from whole territories of Turkey (Coleoptera: Cerambycoidea) part III - Cerambycidae (Dorcadioninae). Munis Entomol Zool. 7:759-779.

Özdikmen H. 2015. A new synonym of Dorcadion (Cribridorcadion) andirinense Bernhauer \& Peks, 2011 (Cerambycidae). Munis Entomol Zool. 10:289-290.

Özdikmen H, Demir H. 2006. Notes on longicorn beetles fauna of Turkey (Coleoptera: Cerambycidae). Munis Entomol Zool. 1:157-166.

Özdikmen H, Güven M, Gören C. 2010. Longhorned beetles fauna of Amanos Mountains, Southern Turkey (Coleoptera: Cerambycidae). Munis Entomol Zool. 5:1141-1167.

Özdikmen H, Hasbenli A. 2004a. Contribution to the knowledge of longhorned bettles (Coleoptera, (erambycidae) from Turkey, Subfamily Lamiinae. J Ent Res Soc. 6:25-49.

Özdikmen H, Hasbenli A. 2004b. A New Subspecies of Dorcadion dimidiatum Motschulsky, 1838 (Coleoptera, Cerambycidae, Lamiinae) from Turkey. J Ent Res Soc. 6:23-27.

Özdikmen H, Kaya G. 2013. Dorcadion (Megalodorcadion) Pesarini \& Sabbadini, 1998 with a new species from Turkey (Coleoptera: Cerambycidae). Munis Entomol Zool. 8:493-501.

Özdikmen H, Kaya G. 2015a. Dorcadion muchei Breuning, 1962 rest. nov. and Dorcadion subatritarse Breuning, 1962 rest. nov. (Cerambycidae). Munis Entomol Zool. 10:272-276.

Özdikmen H, Kaya G. 2015b. Dorcadion (Cribridorcadion) javeti Kraatz, 1873 rest. nov. (Cerambycidae). Munis Entomol Zool. 10:166-168.

Özdikmen H, Kaya G. 2015c. Dorcadion (Cribridorcadion) obsoletum Kraatz, 1873 rest. nov. (Cerambycidae). Munis Entomol Zool. 10:222-224.

Özdikmen H, Kaya G. 2015d. Dorcadion (Cribridorcadion) paracinerarium Breuning, 1974 rest. nov. (Cerambycidae). Munis Entomol Zool. 10:252-254.

Özdikmen H, Kaya G. 2015e. Dorcadion (Cribridorcadion) gebzeense Breuning, 1974 rest. nov. (Cerambycidae). Munis Entomol Zool. 10:186-187.

Özdikmen H, Kaya G. 2015f. Dorcadion (Maculatodorcadion) phrygicum Peks, 1993 stat. nov. (Cerambycidae). Munis Entomol Zool. 10:199-200.

Özdikmen H, Kaya G. 2015g. A propose to new arrangement on some Dorcadionini (Coleoptera: Cerambycidae). Munis Entomol Zool. 10:1-10.

Özdikmen H, Kaya G, Al-Hamadani N. 2014. A new species of Dorcadion Dalman, 1817 (Coleoptera: Cerambycidae) from Turkey. Acta Zoologica Bulgarica. 66:179-180.

Özdikmen H, Koçak Ö. 2013. A new species of Dorcadion Dalman, 1817 from Turkey (Coleoptera: Cerambycidae). Munis Entomol Zool. 8:672-676.

Özdikmen H, Koçak Ö. 2015. A new species of Dorcadion (Cribridorcadion) (Coleoptera: Cerambycidae) in Turkey. Florida Entomol. 98:439-441.

Özdikmen H, Mercan N, Cihan N. 2012a. A new subspecies of Dorcadion halepense (Kraatz, 1873) from South Turkey (Coleoptera: Cerambycidae). Munis Entomol Zool. 7:604-606.

Özdikmen H, Mercan N, Cihan N. 2012b. A new species of Dorcadion Dalman, 1817 from Turkey (Coleoptera: Cerambycidae). Munis Entomol Zool. 7:550-554.

Özdikmen H, Mercan N, Cihan N. 2012c. Male genitaliae of Dorcadion narlianum Özdikmen et al., 2012 and Dorcadion halepense sehitkamilense Özdikmen et al., 2012 (Coleoptera: Cerambycidae). Munis Entomol Zool. 7:1276-1277.

Özdikmen H, Mercan N, Tunç H. 2012d. Longhorned beetles of Düzce province in Turkey (Coleoptera: Cerambycidae). Munis Entomol Zool. 7:714-731.

Özdikmen H, Okutaner AY. 2006. The longhorned beetles fauna (Coleoptera, Cerambycidae) of Kahramanmaraş province. G U J Sci. 19:77-89.

Özdikmen H, Özdemir Y, Turgut S. 2005. Longhorned beetles collection of the Nazife Tuatay Plant Protection Museum, Ankara, Turkey (Coleoptera, Cerambycidae). J Ent Res Soc. 7:1-33.

Özdikmen H, Şenyüz Y. 2015. A new species of Megalodorcadion (Anatolodorcadion) (Coleoptera: Cerambycidae) in Turkey. Florida Entomol. 98:647-650. 
Özdikmen H, Turgut S, Güzel S. 2009. Longhorned beetles of Ankara region in Turkey (Coleoptera: Cerambycidae). Munis Entomol Zool. 4:59-102.

Pallas PS. 1771. Reise durch verschiedene Provinzen des Rußischen Reichs. Erster Theil. St. Petersburg: Kayserliche Academie der Wißenschaften; p. 3-504.

Pallas PS. 1773. Reise durch verschiedene Provinzen des Rußischen Reichs. Zweyter Theil, zweytes Buch vom Jahr 1771. St. Petersburg: Kayserliche Academie der Wißenschaften; p. 369-744.

Peks H. 1992. Eine neue Dorcadion-Art aus Anatolien (Coleoptera: Cerambycidae). Entomologische Zeitschrift (Stuttgart). 102:197-200.

Peks H. 1993. Drei neue Taxa der Gattung Dorcadion aus Anatolien (Coleoptera, Cerambycidae, Lamiinae). Coleoptera, Schwanfelder Coleopterologische Mitteilungen. 1:3-11.

Peks H. 2010. Eine neue Art der Gattung Dorcadion Dalm. aus der Türkei, Umgebung Iznik (Coleoptera, Cerambycidae, Lamiinae). Coleoptera. 14:213-220.

Perissinotto A, Luchini SR. 1966. Coleotteri Raccolti Nel Vicino e Medio Oriente Nota I. Dorcadion Dalm. (Coleoptera, Cerambycidae). Bollettino della Societa Entomologica Italiana. 96:147-149.

Pesarini C, Sabbadini A. 1994. Catalogo dei tipi del Museo Civico di Storia Naturale di Milano X. I tipi dei Coleotteri Cerambicidi. Atti della Società Italiana di Scienze Naturali e del Museo Civico di Storia Naturale di Milano. 133:217-244.

Pesarini C, Sabbadini A. 1998. Osservazioni sistematiche su alcuni Dorcadion della fauna anatolica, con descrizione di 9 nuovi taxa (Coleoptera, Cerambycidae). Annali del Museo civico di Storia naturale di Ferrara. 1:45-61.

Pesarini C, Sabbadini A. 2004. Ricerche sui Dorcadiini di Grecia. I. Le specie del Peloponneso (Coleoptera Cerambycidae). Atti della Società Italiana di Scienze Naturali e del Museo Civico di Storia Naturale in Milano. 145:133-153.

Pesarini C, Sabbadini A. 2007. Ricerche sui Dorcadiini di Grecia. II. Le specie della Grecia centromeridionale e quelle del gruppo di Dorcadion kozanii (Coleoptera Cerambycidae). Atti della Societa Italiana di Scienze Naturali e del Museo Civico di Storia Naturale in Milano. 2007 Gennaio. 148:35-85.

Pesarini C, Sabbadini A. 2008. Ricerche sui Dorcadiini di Grecia. III. Le specie di Neodorcadion Ganglbauer, 1884, quelle del gruppo di Dorcadion ljubetense e descrizione della nuova specie Dorcadion ariannae (Coleoptera Cerambycidae). Atti della Società Italiana di Scienze Naturali e del Museo Civico di Storia Naturale in Milano. 149:109-124.

Pesarini C, Sabbadini A. 2009. Sei nuovi taxa di Cerambycidae della fauna turca e greca (Coleoptera). Annali del Museo Civico di Storia Naturale di Ferrara. 12:15-32.

Pesarini C, Sabbadini A. 2010. Ricerche sui Dorcadionini di Grecia. IV. Le specie della Macedonia e della Tracia (Coleoptera Cerambycidae). Atti della Societa Italiana di Scienze Naturali e del Museo Civico di Storia Naturale in Milano. 151:179-216.

Pesarini C, Sabbadini A. 2011. Note su Cerambycidae di Grecia e Turchia, con descrizione di tre nuove specie e una nuova sottospecie (Coleoptera). Annali del Museo Civico di Storia Naturale di Ferrara. 13:41-59.

Pesarini C, Sabbadini A. 2013. Note su Dorcadion turchi, con descrizione di cinque nuove specie e tre nuove sottospecie (Coleoptera Cerambycidae). Annali del Museo Civico di Storia Naturale di Ferrara. 14:51-64.

Pic M. 1890. Séance du 11 septembre 1889. [Description d'une espèce nouvelle de Longicorne du genre Neodorcadion Gangl. et communication sur Vadonia bitliensis Chevr.]. Annales de la Société Entomologique de France (Paris). 9:clxxv-clxxvi.

Pic M. 1891. Séance du 13 mai 1891. [description d'un Longicorne nouveau]. Bulletin de la Société Entomologique de France (Paris). 60:Ixxvii-Ixxviii.

Pic M. 1892a. Descriptions. L'Échange, Revue Linnéenne. 8:91.

Pic M. 1892b. Séance du 27 janvier 1892. [Observations complémentaires sur mon Neodorcadion Flachneri]. Bulletin de la Société Entomologique de France (Paris). 61:xxvii.

Pic M. 1893. Séance du 27 juillet 1892. [Neodorcadion Flachneri, v. Merkli, var. n.]. Bulletin de la Société Entomologique de France (Paris). 61:cxcvi. 
Pic M. 1894. Descriptions de deux Coléoptères de la Turquie d'Asie. L'Échange, Revue Linnéenne. 10:110-111.

Pic M. 1895a. Descriptions de Longicornes d'Arménie et régions voisines. L'Échange, Revue Linnéenne. 11:38-40.

Pic M. 1895b. [Diagnoses de plusieurs Coléoptères rapportés de Syrie par M. C. Delagrange]. Bulletin de la Société Entomologique de France (Paris). 63:cclxxxiv-cclxxxv.

Pic M. 1897. Voyage de M. Ch. Delagrange dans la Haute Syrie. Liste des Anthicides et supplément aux Longicornes. Annales de la Société entomologique de France (Paris). 66:389-392.

Pic M. 1898. Supplément. Matériaux pour servir à l'étude des Longicornes. 2:54-58.

Pic M. 1900a. Descriptions. Matériaux pour servir à l'étude des Longicornes. 3:11-16.

Pic M. 1900b. Notes diverses. Matériaux pour servir à l'étude des Longicornes. 3:6-7.

Pic M. 1900c. Contribution à l'étude des Longicornes. L'Échange, Revue Linnéenne. 16:81-83.

Pic M. 1901. Descriptions. Matériaux pour servir à l'étude des Longicornes. 3:9-14.

Pic M. 1902a. Description d'un Dorcadion, de la Turquie d'Asie (Col.). Bulletin du Muséum National d'Histoire Naturelle de Paris. 8:93-94.

Pic M. 1902b. Notes diverses et diagnoses. Matériaux pour servir à l'étude des Longicornes. 4:8-11.

Pic M. 1902c. Coléopteres paléarctiques présumes nouveaux. L'Échange, Revue Linnéenne. 18:2527.

Pic M. 1903a. Notes diverses, diagnoses, synonymies. Matériaux pour servir à l'étude des Longicornes. 4:4-9.

Pic M. 1903b. Espèces et variétés nouvelles de Coléoptères [2me article]. L'Échange, Revue Linnéenne. 19:169-171.

Pic M. 1904. Notes diverses et diagnoses. Matériaux pour servir à l'étude des Longicornes. 5:3-6.

Pic M. 1905a. Descriptions abrégées et notes diverses (2e article). L'Échange, Revue Linnéenne. 21:113-115.

Pic M. 1905b. Nouveaux coléoptères d'Algérie \& d'Asie occidentale. L'Échange, Revue Linnéenne. 21:153-156.

Pic M. 1905c. Descriptions et Notes Diverses. Matériaux pour servir à l'étude des Longicornes. 5:515.

Pic M. 1907. Deux nouvelles variétés du "Dorcadion divisum" Germ. L'Échange, Revue Linnéenne. 23:179.

Pic M. 1908. Descriptions ou diagnoses et notes diverses. L'Échange, Revue Linnéenne. 24:2.

Pic M. 1909a. Descriptions ou diagnoses et notes diverses - suite. L'Échange, Revue Linnéenne. 25:121-123.

Pic M. 1909b. Descriptions ou diagnoses et notes diverses - suite. L'Échange, Revue Linnéenne. 25:97-100.

Pic M. 1910. Notes diverses et diagnoses. Matériaux pour servir á l'étude des Longicornes. 7:2-6.

Pic M. 1911. Descriptions ou diagnoses et notes diverses (suite.). L'Échange, Revue Linnéenne. 27:185-186.

Pic M. 1913. Notes diverses, descriptions et diagnoses (suite.). L'Échange, Revue Linnéenne. 29:185-187.

Pic M. 1914. Notes diverses et diagnoses. Matériaux pour servir à l'étude des Longicornes. 9:3-11.

Pic M. 1916. Notes diverses, descriptions et diagnoses (suite.). L'Échange, Revue Linnéenne. 32:2123.

Pic M. 1917a. Notes diverses, descriptions et diagnoses (suite.). L'Échange, Revue Linnéenne. 33:911.

Pic M. 1917b. Notes diverses et diagnoses. Matériaux pour servir à l'étude des Longicornes. 10:310.

Pic M. 1918. Notes diverses, descriptions et diagnoses (suite.). L'Échange, Revue Linnéenne. 34:911.

Pic M. 1926. Notes diverses, descriptions et diagnoses (suite.). L'Échange, Revue Linnéenne. 42:1314.

Pic M. 1927. Notes diverses, descriptions et diagnoses (suite.). L'Échange, Revue Linnéenne. 43:5-7.

Pic M. 1929. Notes diverses, nouveautés. L'Échange, Revue Linnéenne. 45:9-10. 
Pic M. 1931a. Notes diverses, nouveautés. L'Échange, Revue Linnéenne. 47:1-2.

Pic M. 1931b. Notes diverses, nouveautés (suite.). L'Échange, Revue Linnéenne. 47:5-6.

Pic M. 1931c. Notes diverses, nouveautés (suite.). L'Échange, Revue Linnéenne. 47:9-10.

Pic M. 1932. Notes diverses, nouveautés (suite.). L'Échange, Revue Linnéenne. 48:21-22.

Pic M. 1934. Nouveautés asiatiques. Matériaux pour servir à l'étude des Longicornes. 11:33-40.

Pic M. 1937. Notes diverses, nouveautés (suite.). L'Échange, Revue Linnéenne. 53:5-6.

Pic M. 1942a. Opuscula martialis VII. L'Echange, Revue Linnéenne Numéro spécial. 7:1-16.

Pic M. 1942b. Coléoptères du globe (suite). L'Échange, Revue Linnéenne. 58:5-8.

Pic M. 1942c. Opuscula martialia VIII. L'Echange, Revue Linnéenne Numéro spécial. 8:1-13.

Pic M. 1942d. Opuscula martialis VI. L'Echange, Revue Linnéenne. Numéro spécial 6:1-16.

Pic M. 1948. Coléoptères du globe (suite.). L'Échange, Revue Linnéenne. 64:6-16.

Pic T. 1899. Über Dorcadion divisum Germ. und dessen Varietäten. Entomologisches Nachrichtenblatt (Troppau). 25:349-352.

Plavilstshikov NN. 1927. Coleoptera II. Addenda et corrigenda concernant le Coleopterorum Catalogus, parties 73 et 74 (Lamiinae) de Chr. Aurivillius. Paul Lechevalier, Paris. Encyclopédie Entomologique (série B). 1:49-68.

Plavilstshikov NN. 1937. Descriptions des espèces nouvelles de genres Dorcadion Dalm. et Neodorcadion Ganglb. (Coleoptera, Cerambycidae). Sborník Entomologického Oddeliní Národního Musea v Praze. 15:25-34.

Plavilstshikov NN. 1948. A key for Longicorn beetles of Armenia. Erevan. 232 pp. [in Russian].

Plavilstshikov NN. 1951. New species of timber-beetles of Palaearctic fauna (Coleoptera, Cerambycidae). Archives of the Zoological Museum of Moscow State University. 7:113-122.

Plavilstshikov NN. 1958. Faune de I'URSS. Insects Coléptères. V.23 (1). Cerambycidae (P.3). Sousfamille Lamiinae. p.1. Moscou, Leningrad; p. 592.

Poda von Neuhaus N. 1761. Insecta Musei Graecensis, quae in ordines, genera et species Juxta systema naturae Caroli Linnaei digessit. Widmanstad: Graecii. $12+127+18$ pp.

Rapuzzi P, Sama G. 2012. New taxa and new records of longhorn-beetles from Eastern Mediterranean Region (Coleoptera: Cerambycidae). Munis Entomol Zool. 7:663-690.

Reitter E. 1889. Neue Coleopteren aus Europa, den angrenzenden Ländern und Sibirien, mit Bemerkungen über bekannte Arten. Sechster Theil. Deutsche entomologische Zeitschrift. 33:17-44.

Reitter E. 1900. Beitrag zur Coleopteren-Fauna von Europa und den angrenzenden Ländern. Deutsche entomologische Zeitschrift (Berlin). 1900:81-88.

Rejzek M, Hoskovec M. 1999. Cerambycidae of Nemrut Dağı National Park (Anatolia, South-East Turkey). Biocosme Mésogéen (Nice). 15:257-272.

Sabbadini A, Pesarini C. 1992. Due nuove specie dei generi Parmena Latr. e Dorcadion Dalm. appartenenti alla fauna Turca (Coleoptera Cerambycidae). Bollettino Della Società Entomologica Italiana (Genova). 124:27-32.

Sahlberg CR. 1823. Periculi entomographici, species insectorum nondum descriptas propositur. Aboae. 4:46-64.

Sama G. 1982. Contributo allo studio dei coleotteri Cerambycidae di Grecia e Asia Minore. Fragmenta Entomologica (Roma). 16:205-227.

Sama G. 1999. Notes on some Cerambycidae names published by Kraatz and Chevrolat (Coleoptera, Cerambycidae). Entomologische Zeitschrift (Stuttgart). 109:290-295.

Sama G, Dascalu M-M, Pesarini C. 2010. Description of Dorcadion gashtarovi n. sp. (Coleoptera, Cerambycidae) from Romania and Bulgaria with review of the closely related species. NorthWestern J Zool. 6:286-293.

Sama G, Rapuzzi P, Özdikmen H. 2012. Preliminary report of the entomological surveys (2010, 2011) of G. Sama and P. Rapuzzi to Turkey (Coleoptera: Cerambycidae). Munis Entomol Zool. 7:22-45.

Scopoli JA. 1763. Entomologia Carniolica exhibens insecta Carnioliae indigena et distributa in ordines, genera, species, varietates. Methodo Linnaeana, Vindobonae. 1-421.

Şenyüz Y, Özdikmen H. 2013. A contribution to the knowledge of Turkish longicorn beetles fauna (Coleoptera: Cerambycidae). Munis Entomol Zool. 8:571-577. 
Suvorov GL. 1913. Beschreibung neuer Cerambyciden-Arten (Coleoptera). Revue Russe d'Entomologie (Saint-Petersbourg). 13:66-81.

Suvorov GL. 1915. Espèces nouvelles des genres Dorcadion et Compsodorcadion (Coleoptera, Cerambycidae). Revue Russe d'Entomologie (Saint-Petersbourg). 15:115-121.

Swainson W, Shuckard WE. 1840. On the history and natural arrangement of insects. London: Longman, Brown, Green \& Longman's; 406 pp.

Tavakilian G. 2015. Base de données Titan sur les Cerambycidés ou Longicornes [cited 2015 Jan 16]. Available from: http://lis-02.snv.jussieu.fr/titan/

Tekin K, Özdikmen H. 2015. A contribution of Turkish longhorned beetles fauna from Bursa (Coleoptera: Cerambycidae). Munis Entomol Zool. 10:122-130.

Théry A. 1896. Séance du 26 février 1896. Description de quelques Cérambycides paléarctiques [Col.]. Bulletin de la Société Entomologique de France (Paris). 1896:108-110.

Thomson J. 1865. Diagnoses d'espèces nouvelles qui seront décrites dans l'appendix du systema cerambycidarum. Mémoires de la Société Royale des Sciences de Liège. 19:541-578.

Thomson J. 1867. VI. Supplément à la Révision de la sous-tribu des Dorcadionites (P. 10). Physis Recueil d'Histoire Naturelle (Paris). 1:118-125.

Tippmann FF. 1958. Dorcadion-Belustigungen am Leitha-Gebrige (Austria inferior). Mitteilungen der Münchener entomologischen Gessellschaft. 48:130-165.

Tournier H. 1872. Catalogue des Longicornes récoltés par M. Théophile Deyrolle, en Imerétie, Mingrélie et Géorgie, et description des espèces nouvelles. Revue et Magasin de Zoologie pure et appliquée (Paris). 23:276-292.

Tuatay N, Kalkandelen A, Aysev N. 1972. Bitki Koruma Müzesi Böcek Kataloğu (1961-1971). Ankara: T.C. Tarım Bakanlığı; p. 53-55.

Turgut S, Özdikmen H. 2010. New data for Turkish longhorned beetles fauna from Southern Turkey (Coleoptera: Cerambycidae). Munis Entomol Zool. 5:859-889.

Villiers A. 1959. Cérambycides de Turquie. L'Entomologiste. 15:7-11.

Villiers A. 1967. Contribution à la faune de I'Iran. I. - Coléoptères Cerambycidae. Annales de la Société Entomologique de France (NS). 3:327-379.

Vives E. 1976. Contribución al conocimiento de los Iberodorcadion Breuning (Col. Cerambycidae). Miscellanea Zoologica. 111:163-168.

Waltl J. 1838. Beyträge zur Kenntniss der Coleopteren der Türken. Isis Von Oken (Leipzig). 31:449472.

Appendix 1. A checklist of Dorcadionini of Turkey. The endemic species are marked with an asterisk

Tribe DORCADIONINI Swainson and Shuckard, 1840

Genus DORCADION Dalman, 1817: 397

Subgenus CARINATODORCADION Breuning, 1943: 524

D. aethiops Scopoli, 1763: 53 (Cerambyx)

D. aethiops aethiops Scopoli, 1763: 53 (Cerambyx)

D. fulvum Scopoli, 1763: 53 (Cerambyx)

D. fulvum erythropterum Fischer von Waldheim, 1823a: table L-7 (D. erythropteron)

D. hybridum Ganglbauer, 1884: 441

D. hybridum hybridum Ganglbauer, 1884: 441

${ }^{*} D$. ingeae Peks, 1993: 3

Subgenus Cribridorcadion Pic, 1901: 12

${ }^{*} D$. abstersum Holzschuh, 1982: 75

${ }^{*} D$. accola Heyden, 1894: 87

${ }^{*}$ D. afflictum Pesarini and Sabbadini, 1998: 54

${ }^{*} D$. akpinarense Bernhauer and Peks, 2010: 196

${ }^{*}$ D. albolineatum Küster, 1847: 86

${ }^{*}$ D. albonotatum Pic, 1895a: 39 
${ }^{*}$ D. altinyaylaense Bernhauer and Peks, 2014a: 106

${ }^{*} D$. amanense Breuning, 1943: 94

${ }^{*} D$. anatolicum Pic, 1900a: 12

${ }^{*} D$. andirinense Bernhauer and Peks, 2011a: 218

${ }^{*}$ D. apicerufum Breuning, 1943: 91 [RN]

${ }^{*} D$. aracense Bernhauer and Peks, 2011b: 204

${ }^{*}$ D. arcivagum J. Thomson, 1867: 121

${ }^{*} D$. ardahense Breuning, 1975: 10

D. atritarse Pic, 1931a: 10 (D. pedestre var.)

${ }^{*} D$. avgadiense Bernhauer and Peks, 2011a: 215

D. axillare Küster, 1847a: 88

D. axillare axillare Küster, 1847: 88

${ }^{*} D$. bangi Heyden, 1894: 89

${ }^{*} D$. bangi bangi Heyden, 1894: 89

*D. bangi heinzorum Braun, 1975: 17 (D. heinzorum)

${ }^{*} D$. bangi roridum Pesarini and Sabbadini, 1998: 55

${ }^{*}$ D. banjkovskyi Plavilstshikov, 1958: 147

${ }^{*} D$. basale Kraatz, 1873: 86

${ }^{*} D$. beloni Pic, 1891: Ixxvii

${ }^{*} D$. bisignatum Jakovlev, 1899: 66

D. bistriatum Pic, 1898: 55

${ }^{*} D$. bithyniense Chevrolat, 1856: 88

D. blanchardi Mulsant and Rey, 1863: 147

${ }^{*}$ D. blandulum Holzschuh, 1977: 131

${ }^{*} D$. bodemeyeri K. Daniel, 1900: 140

${ }^{*} D$. boluense Breuning, 1962b: 38

${ }^{*} D$. boluense boluense Breuning, 1962b: 38

${ }^{*} D$. boluense corallinum Pesarini and Sabbadini, 1998: 53

${ }^{*} D$. boluense imitator Pesarini and Sabbadini, 1998: 53

${ }^{*} D$. boszdaghense Fairmaire, 1866: 275

${ }^{*} D$. bouilloni Breuning and Ruspoli, 1975: 116

${ }^{*}$ D. brauni Breuning, 1979: 92

${ }^{*} D$. bremeri Breuning, 1981: 181

D. breuningi Heyrovský, 1943: 78

${ }^{*} D$. bulgharmaadense Breuning, 1946: 116

${ }^{*} D$. cachinno J. Thomson, 1867: 120

${ }^{*} D$. caglayanceritense Bernhauer and Peks, 2013a: 314

${ }^{*}$ D. carinipenne Pic, 1900a: 13

${ }^{*}$ D. carolisturanii Breuning and Ruspoli, 1971: 128

D. catenatum Waltl, 1838: 469

D. catenatum catenatum Waltl, 1838: 469

${ }^{*} D$. catenatum dissimile Ganglbauer, 1884: 458 (D. divisum var.)

${ }^{*}$ D. catenatum intercisum Kraatz, 1873: 66 (D. latevittatum var.)

${ }^{*}$ D. catenatum loratum J. Thomson, 1867: 123 (D. loratum)

D. catenatum mytilinense Kraatz, 1873: 66 (D. latevittatum var.)

${ }^{*} D$. catenatum oedemischense Heyrovský, 1932: 104 (D. divisum var.)

${ }^{*}$ D. catenatum subdivisum Breuning, 1955: 263 (D. divisum ssp.)

${ }^{*} D$. cinctellum Fairmaire, 1866: 272

D. cingulatum Ganglbauer, 1884: 484

${ }^{*} D$. coiffaiti Breuning, 1962a: 392

D. condensatum Küster, 1852: 92

${ }^{*} D$. confluens Fairmaire, 1866: 274

${ }^{*}$ D. conspersum Pesarini and Sabbadini, 2013: 56

D. cretosum Ferrari, 1864: 479 
*D. crux Billberg, 1817: 175 (Lamia)

${ }^{*}$ D. culminicola J. Thomson, 1867: 122

${ }^{*} D$. delagrangei Pic, 1894: 110

${ }^{*} D$. deyrollei Ganglbauer, 1884: 482

${ }^{*}$ D. deyrollei deyrollei Ganglbauer, 1884: 482

${ }^{*} D$. deyrollei opacum Pesarini and Sabbadini, 2013: 54

D. dimidiatum Motschulsky, 1838: 186

D. dimidiatum dimidiatum Motschulsky, 1838: 186

${ }^{*} D$. dimidiatum kelkiticum Özdikmen and Hasbenli, 2004b: 23

${ }^{*}$ D. dimidiatum korgei Breuning, 1966a: 21

${ }^{*}$ D. dobrovljanskii Suvorov, 1915: 116

${ }^{*}$ D. drusoides Breuning, 1962a: 375

${ }^{*} D$. eflaniense Bernhauer and Peks, 2011b: 205

${ }^{*}$ D. elazigi Fuchs and Breuning, 1971: 439

${ }^{*} D$. enricisturanii Breuning and Ruspoli, 1971: 127

${ }^{*} D$. enricisturanii densepunctatum Braun, 1978a: 107

${ }^{*} D$. enricisturanii enricisturanii Breuning and Ruspoli, 1971: 127

${ }^{*} D$. erdemi Özdikmen, Kaya and Al-Hamadani, 2014: 179

D. equestre Laxmann, 1770: 596 (Cerambyx)

${ }^{*} D$. equestre bernhauerorum Peks, 2010: 213

D. equestre bisuturale Jureček, 1933: 128 (D. equestre var.)

${ }^{*} D$. equestre festivum Pesarini and Sabbadini, 2013: 58

${ }^{*} D$. equestre nogelli Fairmaire, 1866: 270 (D. nogelli)

${ }^{*}$ D. eskipazarense Bernhauer and Peks, 2011 b: 203

D. ferruginipes Ménétriés, 1836: 151

${ }^{*} D$. formosum Kraatz, 1871: 411

${ }^{*} D$. formosum formosum Kraatz, 1871: 411

*D. formosum ponticum Breuning, 1964a: 1

D. gallipolitanum J. Thomson, 1867: 59

D. gallipolitanum gallipolitanum J. Thomson, 1867: 59

${ }^{*} D$. gebzeense Breuning, 1974a: 148

${ }^{*}$ D. gencense Bernhauer and Peks, 2014a: 110

${ }^{*}$ D. glabricolle Breuning, 1943: 92

${ }^{*}$ D. goelbasiense Bernhauer and Peks, 2013a: 310

${ }^{*}$ D. goelbasiense goelbasiense Bernhauer and Peks, 2013a: 310

${ }^{*} D$. goelbasiense karadutense Bernhauer and Peks, 2013a: 311

${ }^{*}$ D. goelbasiense kathaense Bernhauer and Peks, $2013 \mathrm{a}$ : 312

${ }^{*} D$. goelbasiense oeksuezdagense Bernhauer and Peks, 2013a: 311

${ }^{*} D$. guzeldereense Bernhauer and Peks, 2012: 213

${ }^{*} D$. hacgedigiense Bernhauer and Peks, 2011 a: 216

D. haemorrhoidale Hampe, 1852: 313

D. halepense Kraatz, 1873: 72 (D. saulcyi var.)

D. halepense halepense Kraatz, 1873: 72 (D. saulcyi var.)

${ }^{*} D$. halepense sehitkamilense Özdikmen, Mercan and Cihan, 2012a: 604

${ }^{*} D$. hampii Mulsant and Rey, 1863: 157

${ }^{*} D$. hampii aureovittatum Kraatz, 1873: 81 (D. aureovittatum)

${ }^{*}$ D. hampii hampii Mulsant and Rey, 1863: 157

${ }^{*} D$. hanneloreae Bernhauer and Peks, 2014a: 107

${ }^{*} D$. heinzi Breuning, 1964a: 1 (D. cinerarium ssp.)

D. hellmanni Ganglbauer, 1884: 486

${ }^{*}$ D. holtzi Pic, 1905a: 115 (D. culminicola ssp.)

${ }^{*} D$. iconiense K. Daniel, 1900: 140

${ }^{*} D$. infernale Mulsant and Rey, 1863: 158

${ }^{*} D$. infernale asperatum Breuning, 1947: 169 
${ }^{*} D$. infernale edremitense Breuning, 1966a: 20

${ }^{*} D$. infernale infernale Mulsant and Rey, 1863: 158

${ }^{*} D$. infernale miminfernale Breuning, 1970: 98 (D. miminfernale)

${ }^{*} D$. inikliense Bernhauer and Peks, 2010b: 208

${ }^{*} D$. inopinatum Pesarini and Sabbadini, 2013: 51

${ }^{*} D$. inspersum Holzschuh, 1982: 76

${ }^{*} D$. intrusum Pesarini and Sabbadini, 2009: 26

${ }^{*} D$. ispartense Breuning, 1960: 1

${ }^{*} D$. ispirense Bernhauer and Peks, 2013b: 328

${ }^{*}$ D. ivani Pesarini and Sabbadini, 2011: 47

${ }^{*}$ D. jacovleviellum Plavilstshikov, 1951: 120

*D. janatai Kadlec, 2006: 11

D. javeti Kraatz, 1873: 91

D. johannisfranci Pesarini and Sabbadini, 2007: 40

${ }^{*}$ D. karacaorenense Bernhauer and Peks, 2012: 211

${ }^{*} D$. karamanense Özdikmen and Koçak, 2013: 672

${ }^{*} D$. karsense Suvorov, 1915: 118

${ }^{*}$ D. kartaldagense Bernhauer and Peks, 2013a: 314

D. kasikoporanum Pic, 1902b: 10

${ }^{*} D$. kazanciense Bernhauer and Peks, 2010: 197

${ }^{*}$ D. kindermanni Waltl, 1838: 470

${ }^{*} D$. kizildagense Bernhauer and Peks, 2012: 212

${ }^{*} D$. konradi Bernhauer and Peks, 2012: 210

${ }^{*} D$. kraetschmeri Bernhauer, 1988: 98

${ }^{*} D$. kurdistanum Breuning, 1944: 12

${ }^{*}$ D. kurucanum Holzschuh, 2007: 249

${ }^{*}$ D. ladikanum Braun, 1976a: 173 [RN]

D. laeve Faldermann, 1837: 278

${ }^{*} D$. laeve micula Plavilstshikov, 1937: 26 (D. micula)

${ }^{*} D$. lameerei Théry, 1896: 109

${ }^{*} D$. ledouxi Breuning, 1974b: 65

${ }^{*} D$. linderi Tournier, 1872: 285

D. lineatocolle Kraatz, 1873: 57 (D. femoratum var.)

${ }^{*} D$. lodosi Sabbadini and Pesarini, 1992: 29

${ }^{*} D$. lodosi abanozense Bernhauer and Peks, 2010: 199

${ }^{*}$ D. lodosi lodosi Sabbadini and Pesarini, 1992: 29

${ }^{*}$ D. lohsei Braun, 1976b: 254

${ }^{*} D$. longulum Breuning, 1943: 89

D. lugubre Kraatz, 1873: 41

D. lugubre lugubre Kraatz, 1873: 41

${ }^{*} D$. maceki Holzschuh, 1995: 41

D. margheritae Breuning, 1964b: 32

${ }^{*} D$. martini Bernhauer, 1988: 100

${ }^{*} D$. menradi Holzschuh, 1989: 172

${ }^{*}$ D. merkli Ganglbauer, 1884: 506

D. mesopotamicum Breuning, 1944: 12

${ }^{*} D$. micans J. Thomson, 1867: 61

${ }^{*} D$. micans micans J. Thomson, 1867: 61

${ }^{*} D$. micans susheriense Breuning, 1970: 97 (D. sinopense ssp.)

D. mniszechi Kraatz, 1873: 39

D. mniszechi mniszechi Kraatz, 1873: 39

D. mniszechi cavernosum Lazarev, 2014: 701

${ }^{*} D$. muchei Breuning, 1962b: 38

${ }^{*}$ D. multimaculatum Pic, 1932: 22 
${ }^{*}$ D. murzini Danilevsky, 2015b: 221

${ }^{*} D$. musense Bernhauer and Peks, 2014a: 109

${ }^{*} D$. narlianum Özdikmen, Mercan and Cihan, 2012b: 550

${ }^{*} D$. nigrostriatum Adlbauer, 1982: 104

${ }^{*}$ D. nihalae Rapuzzi and Sama, 2012: 671

${ }^{*}$ D. niksarense Bernhauer and Peks, 2013b: 327

D. nitidum Motschulsky, 1838: 185

${ }^{*} D$. nobile Hampe, 1852: 313

D. nodicorne Tournier, 1872: 287

D. obsoletum Kraatz, 1873: 78 (D. graecum var.)

${ }^{*}$ D. obtusum Breuning, 1944: 13

${ }^{*}$ D. obtusum marashense Breuning, 1948: 59

${ }^{*}$ D. obtusum obtusum Breuning, 1944: 13

${ }^{*} D$. oezdurali Önalp, 1988: 361

${ }^{*}$ D. olympicum Ganglbauer, 1882: 228

${ }^{*}$ D. olympicum convexum Breuning, 1943: 90

${ }^{*}$ D. olympicum flavosuturale Krätschmer, 1987: 340

${ }^{*}$ D. olympicum olympicum Ganglbauer, 1882: 228

${ }^{*} D$. ortrudae Braun, 1978b: 185

${ }^{*} D$. paracinerarium Breuning, 1974a: 149

${ }^{*}$ D. pararufipenne Braun, 1976b: 257

${ }^{*} D$. pararufipenne pararufipenne Braun, 1976b: 257

*D. pararufipenne rassei Braun, 1976b: 261

${ }^{*} D$. parilis Pesarini and Sabbadini, 2013: 52

${ }^{*}$ D. pavesii Pesarini and Sabbadini, 1998: 57

D. pedestre Poda von Neuhaus, 1761: 34 (Cerambyx)

D. pedestre pedestre Poda von Neuhaus, 1761: 34 (Cerambyx)

${ }^{*} D$. peksi Bernhauer, 2010: 190

${ }^{*} D$. pergamenum Pesarini and Sabbadini, 2009: 22

*D. petrovitzi Heyrovský, 1964: 97

D. pilosipenne Breuning, 1943: 90

${ }^{*} D$. pilosipenne kazdagense Özdikmen in Küçükkaykı et al., 2013: 56

${ }^{*}$ D. piochardi Kraatz, 1873: 85

${ }^{*} D$. pittinorum Pesarini and Sabbadini, 1998: 48

${ }^{*}$ D. pluto J. Thomson, 1867: 47

*D. poleti Breuning, 1948: 59

${ }^{*}$ D. postalbosuturale Özdikmen and Koçak, 2015: 439

${ }^{*}$ D. praetermissum Pesarini and Sabbadini, 1998: 47

${ }^{*} D$. praetermissum mikhaili Özdikmen, 2010: 457

${ }^{*} D$. praetermissum praetermissum Pesarini and Sabbadini, 1998: 47

${ }^{*}$ D. preissi Heyden, 1894: 86

${ }^{*}$ D. pseudarcivagum Breuning, 1943: 91

${ }^{*} D$. pseudinfernale Breuning, 1943: 94

${ }^{*} D$. pseudobithyniense Breuning, 1962a: 465

${ }^{*} D$. pseudocinctellum Breuning, 1943: 90

${ }^{*} D$. pseudoholosericeum Breuning, 1962a: 300

${ }^{*} D$. pseudopreissi Breuning, 1962a: 411

D. punctipenne Küster, 1852: 94

${ }^{*}$ D. punctulatum Pesarini and Sabbadini, 2013: 57

${ }^{*} D$. punctulicolle Breuning, 1944: 12

${ }^{*} D$. pygmaeum Breuning, 1947: 168

${ }^{*} D$. quadripustulatum Kraatz, 1873: 88

D. regulare Pic, 1931 b: 6 (D. gallipolitanum var.)

D. regulare regulare Pic, 1931b: 6 (D. gallipolitanum var.) 
${ }^{*}$ D. resadiyeense Bernhauer and Peks, 2011a: 219

${ }^{*} D$. rigattii Breuning, 1966b: 145

${ }^{*} D$. rizeanum Breuning and Villiers, 1967: 60 (Pedestredorcadion)

${ }^{*} D$. robustum Ganglbauer, 1884: 500

${ }^{*} D$. rolandmenradi Peks, 1992: 197

D. rosti Pic, 1900c: 82

${ }^{*}$ D. rufoapicipenne Breuning, 1946: 125

${ }^{*}$ D. sabanoezueense Bernhauer and Peks, 2013b: 326

${ }^{*} D$. sarkislaense Bernhauer and Peks, 2014a: 105

D. saulcyi J. Thomson, 1865: 549

D. scabricolle Dalman, 1817: 174 (Lamia)

${ }^{*} D$. scabricolle balikesirense Breuning, 1962a: 460

${ }^{*} D$. scabricolle caramanicum K. Daniel and J. Daniel, 1903: 332

${ }^{*} D$. scabricolle crassofasciatum Özdikmen in Küçükkaykı et al., 2013: 56

${ }^{*} D$. scabricolle paphlagonicum Breuning, 1962a: 459

D. scabricolle scabricolle Dalman, 1817: 174 (Lamia)

${ }^{*} D$. scabricolle uludaghicum Breuning, 1970: 98

${ }^{*} D$. schultzei Heyden, 1894: 86

${ }^{*}$ D. scrobicolle Kraatz, 1873: 97

${ }^{*} D$. scrobicolle morulum Holzschuh, 1995: 42

${ }^{*} D$. scrobicolle scrobicolle Kraatz, 1873: 97

${ }^{*} D$. sebenense Bernhauer and Peks, 2011b: 202

${ }^{*} D$. semibrunneum Pic, 1903b: 170 (D. mniszechi var.)

${ }^{*}$ D. semibrunneum mediocreimpressum Pic, 1909a: 123 (D. mniszechi var.)

${ }^{*} D$. semibrunneum notatum Pesarini and Sabbadini, 2013: 62

${ }^{*} D$. semibrunneum semibrunneum Pic, 1903b: 170 (D. mniszechi var.)

${ }^{*} D$. semilineatum Fairmaire, 1866: 273

${ }^{*}$ D. semivelutinum Kraatz, 1873: 82

D. septemlineatum Waltl, 1838: 469

${ }^{*}$ D. septemlineatum abanti Braun, 1976c: 54

${ }^{*} D$. septemlineatum demirciense Breuning, 1966a: 19

${ }^{*} D$. septemlineatum novemlineatum Kraatz, 1873: 61

${ }^{*}$ D. septemlineatum octolineatum Kraatz, 1873: 61

D. septemlineatum septemlineatum Waltl, 1838: 469

${ }^{*} D$. sinopense Breuning, 1962a: 299

${ }^{*}$ D. sinuatevittatum Pic, 1937: 6

${ }^{*} D$. skoupyorum Bernhauer and Peks, 2014b: 191 [RN]

D. sodale Hampe, 1852: 313

${ }^{*} D$. sodale blumenthali Breuning, 1966a: 20

D. sodale sodale Hampe, 1852: 313

${ }^{*} D$. sodale trapesunticum Breuning, 1946: 124

${ }^{*} D$. sonjae Peks, 1993: 5

${ }^{*}$ D. steineri Holzschuh, 1977: 129

D. striolatum Kraatz, 1873: 93

D. sturmii Frivaldszky von Frivald, 1837: 179

${ }^{*}$ D. subatritarse Breuning, 1966b: 146

${ }^{*}$ D. subcinctellum Breuning, 1962a: 275

${ }^{*}$ D. subinterruptum Pic, 1900a: 12 (D. divisum var.)

${ }^{*} D$. subsericatum Pic, 1901: 12

${ }^{*} D$. subsericatum majus Breuning, 1962a: 371 (D. rufipenne ssp. major)

${ }^{*} D$. subsericatum rufipenne Breuning, 1946: 118 (D. rufipenne)

${ }^{*}$ D. subsericatum subsericatum Pic, 1901: 12

${ }^{*} D$. subsericatum vulneratum Pesarini and Sabbadini, 1998: 51

${ }^{*}$ D. subvestitum K. Daniel, 1900: 140 
D. sulcipenne Küster, 1847: 87

D. sulcipenne argonauta Suvorov, 1913: 74 (D. argonauta)

${ }^{*} D$. syriense Breuning, 1943: 94

D. tauricum Waltl, 1838: 468

D. tauricum pittinoi Pesarini and Sabbadini, 2010: 185

D. tauricum tauricum Waltl, 1838: 468

${ }^{*} D$. theophilei Pic, 1898: 57

${ }^{*} D$. turcicum Breuning, 1963: 35

${ }^{*}$ D. ullrichi Bernhauer, 1988: 102

D. urmianum Plavilstshikov, 1937: 27

D. variegatum Ganglbauer, 1884: 502

D. wagneri Küster, 1846a: 87

${ }^{*} D$. weyersii Fairmaire, 1866: 271

${ }^{*} D$. yahyaliense Bernhauer and Peks, 2011a: 217

Subgenus Maculatodorcadion Breuning, 1943: 525

${ }^{*} D$. janssensi Breuning, 1966a: 21

${ }^{*}$ D. phrygicum Peks, 1993: 8

D. quadrimaculatum Küster, 1848: 79

${ }^{*} D$. triste Frivaldszky von Frivald, 1845: 184

${ }^{*} D$. wolfi Krätschmer, 1985: 24

Genus Megalodorcadion Pesarini and Sabbadini, 1998: 58

Subgenus Anatolodorcadion Özdikmen and Kaya, 2015g: 3

*M. dombilicoides Özdikmen and Kaya, 2013: 494 (Dorcadion)

*M. glabrofasciatum K. Daniel, 1900: 140 (Dorcadion)

${ }^{*} M$. nudefasciatum Özdikmen and Şenyüz, 2015: 647

Subgenus Fusodorcadion Özdikmen and Kaya, 2015g: 3

${ }^{*}$ M. parallelum Küster, 1847: 79 (Dorcadion)

Subgenus Megalodorcadion Pesarini and Sabbadini, 1998: 58

${ }^{*} M$. escherichi Ganglbauer, 1897: 54 (Dorcadion)

*M. ledereri J. Thomson, 1865: 548 (Dorcadion)

${ }^{*}$ M. walteri Holzschuh, 1991: 55 (Dorcadion)

Genus Neodorcadion Ganglbauer, 1884: 437

Subgenus Neodorcadion Ganglbauer, 1884: 437

N. bilineatum Germar, 1824: 485 (Lamia)

${ }^{*} N$. exornatoides Breuning, 1962a: 68

N. exornatum Frivaldszky von Frivald, 1835: 268 (Dorcadion)

$N$. laqueatum Waltl, 1838: 469 (Dorcadion)

${ }^{*} N$. orientale Ganglbauer, 1884: 510 (N. balcanicum var.)

N. pelleti Mulsant and Rey, 1863: 149 (Dorcadion) 